\title{
Chalcone-based pyridinium salts and their diastereoselective dearomatization to access bi-bridged benzoazepines
}

Le-Le Wang, Hua-Bin Han, Zhao-Hui Cui, Jun-Wei Zhao, Zhan-Wei Bu, and Qi-Lin Wang* Institute of Functional Organic Molecular Engineering, College of Chemistry and Chemical Engineering, Henan University, Kaifeng 475004, China.

E-mail: wangqilin@henu.edu.cn

\section{Table of Contents}

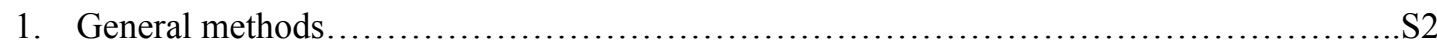

2. Experimental data for novel chalcone-based pyridinium salts1 ........................S2

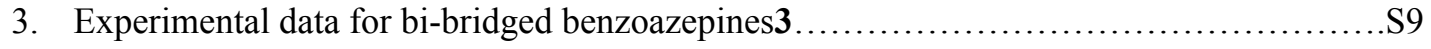

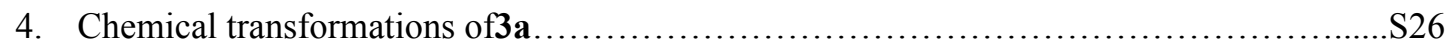

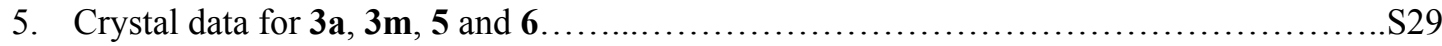

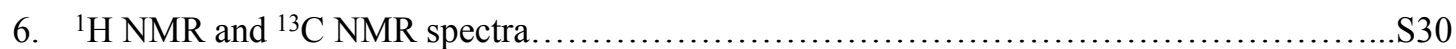




\section{General methods}

NMR spectra were recorded with tetramethylsilane as the internal standard. For most of the samples, ${ }^{1} \mathrm{H}$ NMR spectra were recorded at $400 \mathrm{MHz}$, and ${ }^{13} \mathrm{C}$ NMR spectra were recorded at 100 MHz (Bruker Avance). For others, ${ }^{1} \mathrm{H}$ NMR spectra were recorded at $300 \mathrm{MHz}$, and ${ }^{13} \mathrm{C}$ NMR spectra were recorded at $75 \mathrm{MHz}$ (Bruker Avance). ${ }^{1} \mathrm{H}$ NMR chemical shifts ( $\delta$ ) are reported in ppm relative to tetramethylsilane (TMS) with the solvent signal as the internal standard $\left(\mathrm{CDCl}_{3}\right.$ at $7.26 \mathrm{ppm},\left(\mathrm{CD}_{3}\right)_{2} \mathrm{SO}$ at $\left.2.50 \mathrm{ppm}\right) .{ }^{13} \mathrm{C}$ NMR chemical shifts are reported in ppm from tetramethylsilane (TMS) with the solvent resonance as the internal standard $\left(\mathrm{CDCl}_{3}\right.$ at $77.00 \mathrm{ppm}$, $\left(\mathrm{CD}_{3}\right)_{2} \mathrm{SO}$ at $\left.39.52 \mathrm{ppm}\right)$. Data are given as: s (singlet), d (doublet), t (triplet), q (quartet), dd (double of doublet), br (broad) or m (multiplets), coupling constants $(\mathrm{Hz})$ and integration. Flash column chromatography was carried out using silica gel eluting with ethyl acetate and petroleum ether. High resolution mass spectra were obtained with the Q-TOF-Premier mass spectrometer. Reactions were monitored by TLC and visualized with ultraviolet light.IR spectra were recorded on a Thermo Fisher Nicolet Avatar 360 FTIR spectrometer on a $\mathrm{KBr}$ beam splitter. All the solvents were used directly without any purification.

\section{Experimental data for novel chalcone-based pyridinium salts 1}

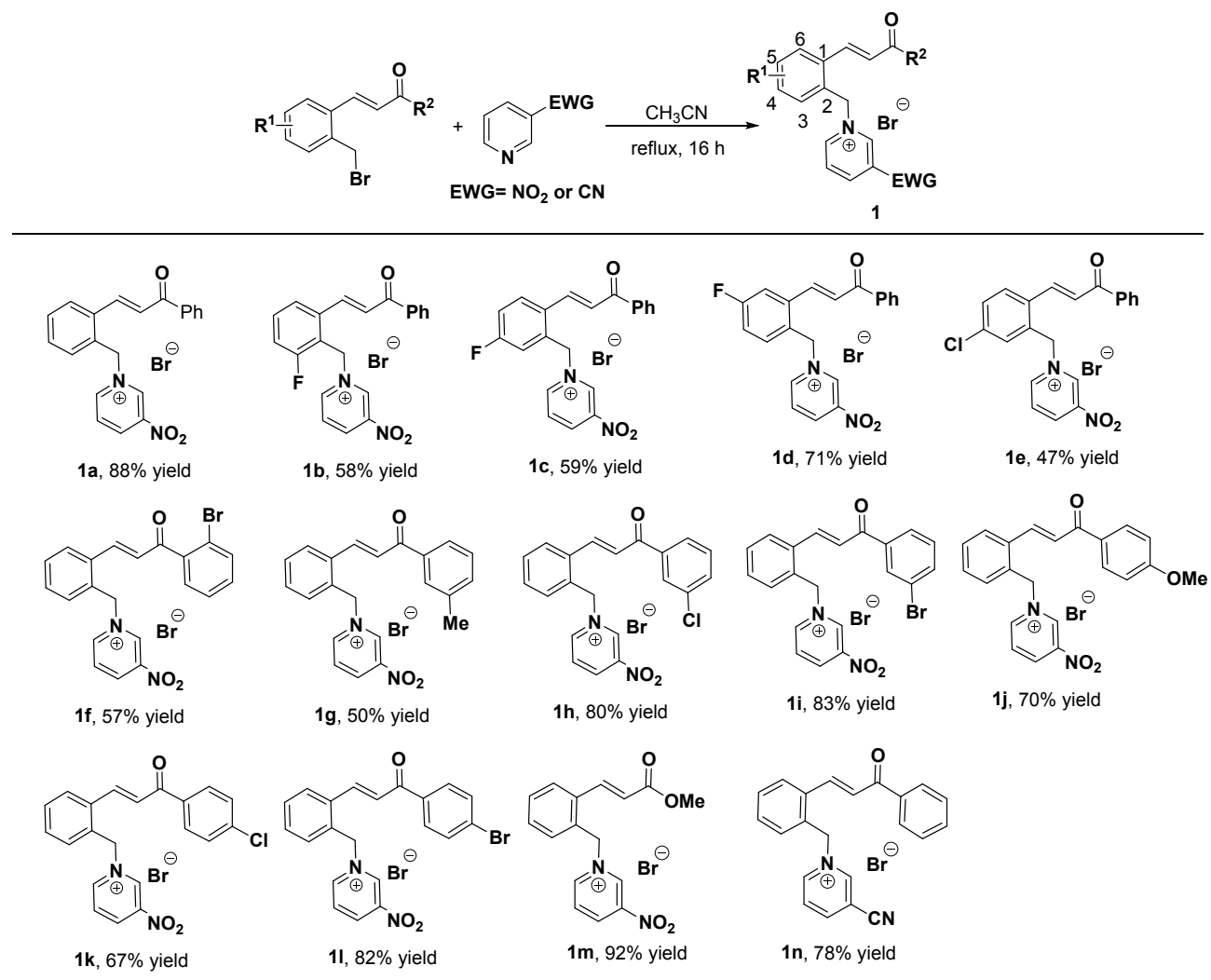


General procedure: A solution of ortho-bromomethyl chalcones (1.5 equiv.) and 3-nitro or 3-cyanopyridine (1.0 equiv.) in $\mathrm{CH}_{3} \mathrm{CN}$ was placed in a Dean-Stark apparatus and the mixture was heated to reflux in oil bath for $16 \mathrm{~h}$. During the reaction process, much precipitate was generated. To purify them, a simple filtration was needed, affording chalcone-based pyridinium salts $\mathbf{1}$ in $47-92 \%$ yields.

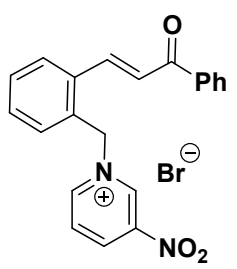

(E)-3-nitro-1-(2-(3-oxo-3-phenylprop-1-en-1-yl)benzyl)pyridin-1-ium bromide (1a)

According to the general procedure, $\mathbf{1 a}$ was prepared on a scale of 8.0 mmol. Yellow solids, 3.00 $\mathrm{g}, 88 \%$ isolated yield obtained by filtration of the precipitate; Reaction time $=16 \mathrm{~h}$; m. p. 210.5-211.4 ${ }^{\circ} \mathrm{C} ;{ }^{1} \mathrm{H}$ NMR (400 MHz, DMSO-d 6 ) $\delta 10.25(\mathrm{~s}, 1 \mathrm{H}), 9.41(\mathrm{~d}, J=8.0 \mathrm{~Hz}, 1 \mathrm{H}), 9.37(\mathrm{~d}$, $J=8.0 \mathrm{~Hz}, 1 \mathrm{H}), 8.47\left(\mathrm{dd}, J_{l}=J_{2}=8.0 \mathrm{~Hz}, 1 \mathrm{H}\right), 8.17-8.14(\mathrm{~m}, 3 \mathrm{H}), 8.08(\mathrm{~d}, J=16.0 \mathrm{~Hz}, 1 \mathrm{H}), 7.88$ (d, $J=16.0 \mathrm{~Hz}, 1 \mathrm{H}), 7.70(\mathrm{t}, J=8.0 \mathrm{~Hz}, 1 \mathrm{H}), 7.61-7.50(\mathrm{~m}, 4 \mathrm{H}), 7.22$ (d, $J=8.0 \mathrm{~Hz}, 1 \mathrm{H}), 6.43(\mathrm{~s}$, $2 \mathrm{H}) ;{ }^{13} \mathrm{C}$ NMR (100 MHz, DMSO- $\left.d_{6}\right) \delta 189.0,149.7,146.6,142.9,140.6,139.1,137.1,134.0$, 133.5, 133.0, 130.8, 129.7, 129.2, 129.1, 128.9, 128.7, 128.2, 126.0, 61.4. IR (KBr) v 3421, 3021, $1655,1599,1547,1348,758 \mathrm{~cm}^{-1}$. HRMS (ESI) calcd. for $\mathrm{C}_{21} \mathrm{H}_{17} \mathrm{~N}_{2} \mathrm{O}_{3}[\mathrm{M}-\mathrm{Br}]^{+}: 345.1234$, found: 345.1232 .<smiles>O=C(/C=C/c1ccccc1F)c1cccc([N+](=O)[O-])c1</smiles>

(E)-1-(2-fluoro-6-(3-oxo-3-phenylprop-1-en-1-yl)benzyl)-3-nitropyridin-1-ium (1b)

According to the general procedure, $\mathbf{1 b}$ was prepared on a scale of $3.10 \mathrm{mmol}$. Yellow solids, 0.80 $\mathrm{g}, 58 \%$ isolated yield obtained by filtration of the precipitate; Reaction time $=16 \mathrm{~h}$; m. p. 164.5-165.3 ${ }^{\circ} \mathrm{C} ;{ }^{1} \mathrm{H}$ NMR (400 MHz, DMSO- $\left.d_{6}\right) \delta 10.15(\mathrm{~s}, 1 \mathrm{H}), 9.36\left(\mathrm{dd}, J_{I}=J_{2}=4.0 \mathrm{~Hz}, 1 \mathrm{H}\right)$, $9.23(\mathrm{~d}, J=8.0 \mathrm{~Hz}, 1 \mathrm{H}), 8.39\left(\mathrm{dd}, J_{1}=J_{2}=8.0 \mathrm{~Hz}, 1 \mathrm{H}\right), 8.17-8.12(\mathrm{~m}, 3 \mathrm{H}), 8.05(\mathrm{~d}, J=8.0 \mathrm{~Hz}$, 1H), $7.93(\mathrm{~d}, J=16.0 \mathrm{~Hz}, 1 \mathrm{H}), 7.72-7.68(\mathrm{~m}, 2 \mathrm{H}), 7.59(\mathrm{t}, J=8.0 \mathrm{~Hz}, 2 \mathrm{H}), 7.48$ (t, $J=8.0 \mathrm{~Hz}$, $1 \mathrm{H}), 6.40(\mathrm{~s}, 2 \mathrm{H}) ;{ }^{13} \mathrm{C}$ NMR $\left(100 \mathrm{MHz}, \mathrm{DMSO}-d_{6}\right) \delta 188.9,161.6\left(\mathrm{~d},{ }^{1} J_{\mathrm{C}-\mathrm{F}}=247.0 \mathrm{~Hz}, 1 \mathrm{C}\right)$, 
149.1, 146.2, 142.2, 140.7, 138.2, 137.8, 137.0, 133.6, 132.6 (d, $J=10.0 \mathrm{~Hz}, 1 \mathrm{C}), 129.2,128.9$, 128.7, 127.9, 124.5, $118.8\left(\mathrm{~d},{ }^{2} J_{\mathrm{C}-\mathrm{F}}=14.0 \mathrm{~Hz}, 1 \mathrm{C}\right), 117.4\left(\mathrm{~d},{ }^{2} J_{\mathrm{C}-\mathrm{F}}=22.0 \mathrm{~Hz}, 1 \mathrm{C}\right), 55.3 . \mathrm{IR}(\mathrm{KBr})$ $v$ 3443, 3019, 1654, 1597, 1549, 1243, $707 \mathrm{~cm}^{-1}$. HRMS (ESI) calcd. for $\mathrm{C}_{21} \mathrm{H}_{16} \mathrm{FN}_{2} \mathrm{O}_{3}$ [M-Br] $]^{+}$: 363.1139, found: 363.1137 .

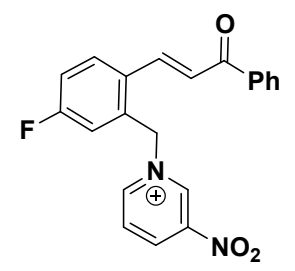

(E)-1-(5-fluoro-2-(3-oxo-3-phenylprop-1-en-1-yl)benzyl)-3-nitropyridin-1-ium (1c)

According to the general procedure, $1 \mathrm{c}$ was prepared on a scale of $2.50 \mathrm{mmol}$. Yellow solids, 0.65 g, $59 \%$ isolated yield obtained by filtration of the precipitate; Reaction time $=16 \mathrm{~h} ; \mathrm{m} . \mathrm{p}$. 171.3-172.1 ${ }^{\circ} \mathrm{C} ;{ }^{1} \mathrm{H}$ NMR (400 MHz, DMSO- $\left.d_{6}\right) \delta 10.27(\mathrm{~s}, 1 \mathrm{H}), 9.44-9.39(\mathrm{~m}, 2 \mathrm{H}), 8.48\left(\mathrm{dd}, J_{l}=\right.$ $\left.J_{2}=8.0 \mathrm{~Hz}, 1 \mathrm{H}\right), 8.25\left(\mathrm{dd}, J_{1}=J_{2}=4.0 \mathrm{~Hz}, 1 \mathrm{H}\right), 8.17\left(\mathrm{dd}, J_{l}=J_{2}=4.0 \mathrm{~Hz}, 2 \mathrm{H}\right), 8.02(\mathrm{~d}, J=12.0$ Hz, 1H), 7.89 (d, $J=12.0 \mathrm{~Hz}, 1 \mathrm{H}), 7.72-7.68(\mathrm{~m}, 1 \mathrm{H}), 7.59$ (t, $J=8.0 \mathrm{~Hz}, 2 \mathrm{H}), 7.45-7.41(\mathrm{~m}, 1 \mathrm{H})$, $7.08\left(\mathrm{dd}, J_{1}=J_{2}=4.0 \mathrm{~Hz}, 1 \mathrm{H}\right), 6.44(\mathrm{~s}, 2 \mathrm{H}) ;{ }^{13} \mathrm{C} \mathrm{NMR}\left(100 \mathrm{MHz}, \mathrm{DMSO}-d_{6}\right) \delta 188.9,163.2(\mathrm{~d}$, $\left.{ }^{1} J_{\mathrm{C}-\mathrm{F}}=248.0 \mathrm{~Hz}, 1 \mathrm{C}\right), 149.8,146.7,143.2,140.7,138.0,137.1,135.7\left(\mathrm{~d},{ }^{3} J_{\mathrm{C}-\mathrm{F}}=7.0 \mathrm{~Hz}, 1 \mathrm{C}\right)$, 133.5, $130.8\left(\mathrm{~d},{ }^{3} J_{\mathrm{C}-\mathrm{F}}=7.0 \mathrm{~Hz}, 1 \mathrm{C}\right), 130.3\left(\mathrm{~d},{ }^{3} J_{\mathrm{C}-\mathrm{F}}=7.0 \mathrm{~Hz}, 1 \mathrm{C}\right), 129.3,128.9,128.7,125.7$, $116.6\left(\mathrm{~d},{ }^{2} J_{\mathrm{C}-\mathrm{F}}=22.0 \mathrm{~Hz}, 1 \mathrm{C}\right), 115.9\left(\mathrm{~d},{ }^{2} J_{\mathrm{C}-\mathrm{F}}=22.0 \mathrm{~Hz}, 1 \mathrm{C}\right), 60.9 . \mathrm{IR}(\mathrm{KBr}) v 3441,3067,2943$, 1653, 1594, 1550, 1220, $718 \mathrm{~cm}^{-1}$. HRMS (ESI) calcd. for $\mathrm{C}_{21} \mathrm{H}_{16} \mathrm{FN}_{2} \mathrm{O}_{3}[\mathrm{M}-\mathrm{Br}]^{+}: 363.1139$, found: 363.1138 .<smiles></smiles>

(E)-1-(4-fluoro-2-(3-oxo-3-phenylprop-1-en-1-yl)benzyl)-3-nitropyridin-1-ium (1d)

According to the general procedure, $\mathbf{1 d}$ was prepared on a scale of $2.50 \mathrm{mmol}$. Yellow solids, 0.79 $\mathrm{g}, 71 \%$ isolated yield obtained by filtration of the precipitate; Reaction time $=16 \mathrm{~h} ; \mathrm{m} . \mathrm{p}$. 174.2-174.8 ${ }^{\circ} \mathrm{C} ;{ }^{1} \mathrm{H}$ NMR (400 MHz, DMSO- $\left.d_{6}\right) \delta 10.22(\mathrm{~s}, 1 \mathrm{H}), 9.40-9.35(\mathrm{~m}, 2 \mathrm{H}), 8.47-8.43(\mathrm{~m}$, $1 \mathrm{H}), 8.18(\mathrm{t}, J=4.0 \mathrm{~Hz}, 2 \mathrm{H}), 8.10\left(\mathrm{dd}, J_{l}=J_{2}=4.0 \mathrm{~Hz}, 1 \mathrm{H}\right), 8.02(\mathrm{~d}, J=16.0 \mathrm{~Hz}, 1 \mathrm{H}), 7.96(\mathrm{~d}, J=$ $16.0 \mathrm{~Hz}, 1 \mathrm{H}), 7.70(\mathrm{t}, J=8.0 \mathrm{~Hz}, 1 \mathrm{H}), 7.59$ (t, $J=8.0 \mathrm{~Hz}, 2 \mathrm{H}), 7.48-7.38(\mathrm{~m}, 2 \mathrm{H}), 6.41$ (d, $J=4.0$ $\mathrm{Hz}, 2 \mathrm{H}) ;{ }^{13} \mathrm{C}$ NMR $\left(100 \mathrm{MHz}, \mathrm{DMSO}-d_{6}\right) \delta 188.9,162.7\left(\mathrm{~d},{ }^{1} J_{\mathrm{C}-\mathrm{F}}=246.0 \mathrm{~Hz}, 1 \mathrm{C}\right), 149.5,146.5$, 
$142.7,140.6,137.7,137.0,136.9,133.6,132.4\left(\mathrm{~d},{ }^{3} J_{\mathrm{C}-\mathrm{F}}=9.0 \mathrm{~Hz}, 1 \mathrm{C}\right), 129.2,128.9\left(\mathrm{~d},{ }^{4} J_{\mathrm{C}-\mathrm{F}}=3.0\right.$ $\mathrm{Hz}, 1 \mathrm{C}), 128.9,128.7,127.2,117.5\left(\mathrm{~d},{ }^{2} J_{\mathrm{C}-\mathrm{F}}=22.0 \mathrm{~Hz}, 1 \mathrm{C}\right), 114.7\left(\mathrm{~d},{ }^{2} J_{\mathrm{C}-\mathrm{F}}=22.0 \mathrm{~Hz}, 1 \mathrm{C}\right), 60.8$. IR $(\mathrm{KBr}) v 3442,2998,1655,1605,1544,1351,1214,726 \mathrm{~cm}^{-1}$. HRMS (ESI) calcd. for $\mathrm{C}_{21} \mathrm{H}_{16} \mathrm{FN}_{2} \mathrm{O}_{3}[\mathrm{M}-\mathrm{Br}]^{+}: 363.1139$, found: 363.1137 .<smiles></smiles>

(E)-1-(5-chloro-2-(3-oxo-3-phenylprop-1-en-1-yl)benzyl)-3-nitropyridin-1-ium (1e)

According to the general procedure, 1e was prepared on a scale of $2.88 \mathrm{mmol}$. Yellow solids, $0.61 \mathrm{~g}, 47 \%$ isolated yield obtained by filtration of the precipitate; Reaction time $=16 \mathrm{~h} ; \mathrm{m} . \mathrm{p}$. 157.7-158.6 ${ }^{\circ} \mathrm{C} ;{ }^{1} \mathrm{H}$ NMR (400 MHz, DMSO- $\left.d_{6}\right) \delta 10.28(\mathrm{~s}, 1 \mathrm{H}), 9.41\left(\mathrm{dd}, J_{I}=J_{2}=4.0 \mathrm{~Hz}, 2 \mathrm{H}\right)$, $8.47\left(\mathrm{dd}, J_{1}=J_{2}=4.0 \mathrm{~Hz}, 1 \mathrm{H}\right), 8.18\left(\mathrm{dd}, J_{1}=J_{2}=8.0 \mathrm{~Hz}, 3 \mathrm{H}\right), 8.02(\mathrm{~d}, J=16.0 \mathrm{~Hz}, 1 \mathrm{H}), 7.91(\mathrm{~d}$, $J=16.0 \mathrm{~Hz}, 1 \mathrm{H}), 7.70(\mathrm{t}, J=8.0 \mathrm{~Hz}, 1 \mathrm{H}), 7.65-7.57(\mathrm{~m}, 3 \mathrm{H}), 7.37$ (d, $J=4.0 \mathrm{~Hz}, 1 \mathrm{H}), 6.44(\mathrm{~s}$, 2H); ${ }^{13} \mathrm{C}$ NMR (100 MHz, DMSO-d $\left.d_{6}\right) \delta 188.9,149.7,146.6,143.2,140.7,137.9,137.0,135.3$, $134.9,133.5,133.0,130.0,129.7,129.2,128.9,128.7,126.5,60.8$, one carbon missing in the aromatic region. IR $(\mathrm{KBr}) v 3448,2929,1648,1601,776 \mathrm{~cm}^{-1}$. HRMS (ESI) calcd. for $\mathrm{C}_{21} \mathrm{H}_{16} \mathrm{ClN}_{2} \mathrm{O}_{3}[\mathrm{M}-\mathrm{Br}]^{+}: 379.0844$, found: 379.0848 .

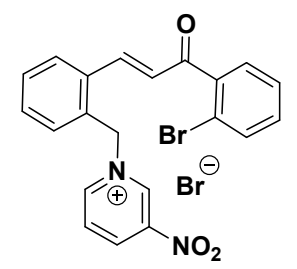

(E)-1-(2-(3-(2-bromophenyl)-3-oxoprop-1-en-1-yl)benzyl)-3-nitropyridin-1-ium bromide (1f)

According to the general procedure, $1 \mathbf{f}$ was prepared on a scale of $2.59 \mathrm{mmol}$. Yellow solids, 0.74 g, $57 \%$ isolated yield obtained by filtration of the precipitate; Reaction time $=16 \mathrm{~h} ; \mathrm{m}$. p. 131.2-133.4 ${ }^{\circ} \mathrm{C} ;{ }^{1} \mathrm{H}$ NMR (400 MHz, DMSO- $\left.d_{6}\right) \delta 10.17$ (s, $\left.1 \mathrm{H}\right), 9.44\left(\mathrm{dd}, J_{l}=J_{2}=4.0 \mathrm{~Hz}, 1 \mathrm{H}\right)$, $9.32(\mathrm{~d}, J=4.0 \mathrm{~Hz}, 1 \mathrm{H}), 8.48\left(\mathrm{dd}, J_{I}=J_{2}=8.0 \mathrm{~Hz}, 1 \mathrm{H}\right), 7.99\left(\mathrm{dd}, J_{I}=J_{2}=4.0 \mathrm{~Hz}, 1 \mathrm{H}\right), 7.80(\mathrm{~d}$, $J=16.0 \mathrm{~Hz}, 1 \mathrm{H}), 7.75\left(\mathrm{dd}, J_{1}=J_{2}=4.0 \mathrm{~Hz}, 1 \mathrm{H}\right), 7.61-7.47(\mathrm{~m}, 5 \mathrm{H}), 7.25(\mathrm{~d}, J=16.0 \mathrm{~Hz}, 1 \mathrm{H})$, $7.16(\mathrm{~d}, J=8.0 \mathrm{~Hz}, 1 \mathrm{H}), 6.34(\mathrm{~s}, 2 \mathrm{H}) ;{ }^{13} \mathrm{C}$ NMR $\left(100 \mathrm{MHz}, \mathrm{DMSO}-d_{6}\right) \delta 193.0,149.7,146.6$, $142.8,140.7,140.6,140.2,133.4,133.0,132.4,131.1,129.8,129.6,129.4,129.3,129.0,128.1$, 128.0, 118.8, 61.3, one carbon missing in the aromatic region. IR $(\mathrm{KBr}) v 3440,3010,1615,1545$, 
1356, $746 \mathrm{~cm}^{-1}$. HRMS (ESI) calcd. for $\mathrm{C}_{21} \mathrm{H}_{16} \mathrm{BrN}_{2} \mathrm{O}_{3}$ [M-Br] $]^{+}$: 423.0339, found: 423.0340 .

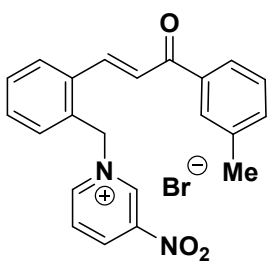

(E)-3-nitro-1-(2-(3-oxo-3-( $m$-tolyl)prop-1-en-1-yl)benzyl)pyridin-1-ium bromide (1g)

According to the general procedure, $1 \mathrm{~g}$ was prepared on a scale of $2.60 \mathrm{mmol}$. Yellow solids, 0.57

g, $50 \%$ isolated yield obtained by filtration of the precipitate; Reaction time $=16 \mathrm{~h} ; \mathrm{m} . \mathrm{p}$. 190.2-191. $0^{\circ} \mathrm{C} ;{ }^{1} \mathrm{H}$ NMR $\left(400 \mathrm{MHz}, \mathrm{DMSO}-d_{6}\right) \delta 10.26(\mathrm{~s}, 1 \mathrm{H}), 9.43-9.38(\mathrm{~m}, 2 \mathrm{H}), 8.49\left(\mathrm{dd}, J_{l}=\right.$ $\left.J_{2}=4.0 \mathrm{~Hz}, 1 \mathrm{H}\right), 8.16(\mathrm{~d}, J=8.0 \mathrm{~Hz}, 1 \mathrm{H}), 8.07(\mathrm{~d}, J=16.0 \mathrm{~Hz}, 1 \mathrm{H}), 7.99(\mathrm{~s}, 1 \mathrm{H}), 7.96(\mathrm{~d}, J=8.0$ Hz, 1H), $7.87(\mathrm{~d}, J=16.0 \mathrm{~Hz}, 1 \mathrm{H}), 7.59-7.45(\mathrm{~m}, 4 \mathrm{H}), 7.24(\mathrm{~d}, J=8.0 \mathrm{~Hz}, 1 \mathrm{H}), 6.45(\mathrm{~s}, 2 \mathrm{H}), 2.42$ (s, 3H); ${ }^{13} \mathrm{C}$ NMR (100 MHz, DMSO- $\left.d_{6}\right) \delta 189.0,149.7,146.6,142.8,140.6,138.9,138.3,137.2$, 134.1, 134.0, 132.9, 130.8, 129.7, 129.3, 129.2, 129.1, 128.8, 128.2, 126.1, 125.9, 61.4, 20.9. IR (KBr) $v$ 3441, 1648, 1354, $765 \mathrm{~cm}^{-1}$. HRMS (ESI) calcd. for $\mathrm{C}_{22} \mathrm{H}_{19} \mathrm{~N}_{2} \mathrm{O}_{3}[\mathrm{M}-\mathrm{Br}]^{+}: 359.1390$, found: 359.1390 .

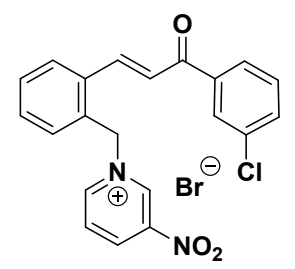

(E)-1-(2-(3-(3-chlorophenyl)-3-oxoprop-1-en-1-yl)benzyl)-3-nitropyridin-1-ium bromide (1h)

According to the general procedure, $\mathbf{1 h}$ was prepared on a scale of $2.80 \mathrm{mmol}$. Yellow solids, $1.03 \mathrm{~g}, 80 \%$ isolated yield obtained by filtration of the precipitate; Reaction time $=16 \mathrm{~h} ; \mathrm{m} . \mathrm{p}$. 171.3-171.8 ${ }^{\circ} \mathrm{C} ;{ }^{1} \mathrm{H}$ NMR $\left(400 \mathrm{MHz}, \mathrm{DMSO}-d_{6}\right) \delta 10.24(\mathrm{~s}, 1 \mathrm{H}), 9.43-9.37(\mathrm{~m}, 2 \mathrm{H}), 8.47\left(\mathrm{dd}, J_{l}=\right.$ $\left.J_{2}=4.0 \mathrm{~Hz}, 1 \mathrm{H}\right), 8.20-8.18(\mathrm{~m}, 2 \mathrm{H}), 8.14-8.09(\mathrm{~m}, 2 \mathrm{H}), 7.90(\mathrm{~d}, J=16.0 \mathrm{~Hz}, 1 \mathrm{H}), 7.76\left(\mathrm{dd}, J_{l}=J_{2}\right.$ $=4.0 \mathrm{~Hz}, 1 \mathrm{H}), 7.64-7.51(\mathrm{~m}, 3 \mathrm{H}), 7.25(\mathrm{~d}, J=8.0 \mathrm{~Hz}, 1 \mathrm{H}), 6.44(\mathrm{~s}, 2 \mathrm{H}) ;{ }^{13} \mathrm{C}$ NMR $(100 \mathrm{MHz}$, DMSO- $\left.d_{6}\right) \delta 187.8,149.7,146.6,142.8,140.6,139.8,139.0,133.9,133.8,133.2,133.0,131.0$, $130.9,129.7,129.2,129.2,128.3,128.2,127.3,125.5,61.4$. IR (KBr) v 3440, 3010, 1653, 1602 , 1552, 1354, $764 \mathrm{~cm}^{-1}$. HRMS (ESI) calcd. for $\mathrm{C}_{21} \mathrm{H}_{16} \mathrm{ClN}_{2} \mathrm{O}_{3}[\mathrm{M}-\mathrm{Br}]^{+}: 379.0844$, found: 379.0843 . 


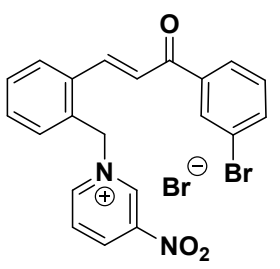

(E)-1-(2-(3-(3-bromophenyl)-3-oxoprop-1-en-1-yl)benzyl)-3-nitropyridin-1-ium bromide (1i)

According to the general procedure, 1i was prepared on a scale of $3.00 \mathrm{mmol}$. Yellow solids, 1.24 g, $83 \%$ isolated yield obtained by filtration of the precipitate; Reaction time $=16 \mathrm{~h} ; \mathrm{m} . \mathrm{p}$. 169.3-170.2 ${ }^{\circ} \mathrm{C} ;{ }^{1} \mathrm{H}$ NMR (400 MHz, DMSO- $\left.d_{6}\right) \delta 10.24(\mathrm{~s}, 1 \mathrm{H}), 9.41(\mathrm{~d}, J=8.0 \mathrm{~Hz}, 1 \mathrm{H}), 9.37(\mathrm{~d}$, $J=8.0 \mathrm{~Hz}, 1 \mathrm{H}), 8.47\left(\mathrm{dd}, J_{1}=J_{2}=8.0 \mathrm{~Hz}, 1 \mathrm{H}\right), 8.32(\mathrm{~s}, 1 \mathrm{H}), 8.18(\mathrm{t}, J=8.0 \mathrm{~Hz}, 2 \mathrm{H}), 8.11(\mathrm{~d}, J=$ $12.0 \mathrm{~Hz}, 1 \mathrm{H}), 7.91(\mathrm{~d}, J=8.0 \mathrm{~Hz}, 1 \mathrm{H}), 7.88(\mathrm{~s}, 1 \mathrm{H}), 7.58-7.53(\mathrm{~m}, 3 \mathrm{H}), 7.24(\mathrm{~d}, J=8.0 \mathrm{~Hz}, 1 \mathrm{H})$, $6.44(\mathrm{~s}, 2 \mathrm{H}) ;{ }^{13} \mathrm{C}$ NMR (100 MHz, DMSO-d $) \delta 187.8,149.7,146.6,142.9,140.6,139.8,139.2$, $136.1,133.8,133.1,131.1,131.1,131.0,129.7,129.2,129.1,128.3,127.7,125.5,122.4,61.4$. IR $(\mathrm{KBr}) v 3445,3012,1653,1615,1549,768 \mathrm{~cm}^{-1}$. HRMS (ESI) calcd. for $\mathrm{C}_{21} \mathrm{H}_{16} \mathrm{BrN}_{2} \mathrm{O}_{3}[\mathrm{M}-\mathrm{Br}]^{+}$: 423.0339, found: 423.0334.

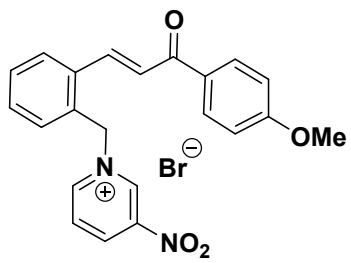

(E)-1-(2-(3-(4-methoxyphenyl)-3-oxoprop-1-en-1-yl)benzyl)-3-nitropyridin-1-ium bromide (1j)

According to the general procedure, $\mathbf{1 j}$ was prepared on a scale of $2.40 \mathrm{mmol}$. Yellow solids, 0.76 $\mathrm{g}, 70 \%$ isolated yield obtained by filtration of the precipitate; Reaction time $=16 \mathrm{~h} ; \mathrm{m}$. $\mathrm{p}$. $172.8-173.4^{\circ} \mathrm{C} ;{ }^{1} \mathrm{H}$ NMR $\left(400 \mathrm{MHz}, \mathrm{DMSO}_{-}\right)_{6} \delta 10.26(\mathrm{~s}, 1 \mathrm{H}), 9.43-9.37(\mathrm{~m}, 2 \mathrm{H}), 8.47\left(\mathrm{dd}, J_{1}=\right.$ $\left.J_{2}=8.0 \mathrm{~Hz}, 1 \mathrm{H}\right), 8.17(\mathrm{~d}, J=8.0 \mathrm{~Hz}, 2 \mathrm{H}), 8.14(\mathrm{~d}, J=8.0 \mathrm{~Hz}, 1 \mathrm{H}), 8.03(\mathrm{~d}, J=16.0 \mathrm{~Hz}, 1 \mathrm{H}), 7.87$ $(\mathrm{d}, J=16.0 \mathrm{~Hz}, 1 \mathrm{H}), 7.58-7.48(\mathrm{~m}, 2 \mathrm{H}), 7.24(\mathrm{~d}, J=8.0 \mathrm{~Hz}, 1 \mathrm{H}), 7.10(\mathrm{~d}, J=12.0 \mathrm{~Hz}, 2 \mathrm{H}), 6.44$ (s, 2H), $3.87(\mathrm{~s}, 3 \mathrm{H}) ;{ }^{13} \mathrm{C}$ NMR (100 MHz, DMSO-d 6$) \delta 187.2,163.4,149.7,146.5,142.9,140.6$, $138.2,134.2,132.8,131.1,130.6,130.0,129.7,129.2,129.1,128.2,126.0,114.1,61.5,55.7$. IR $(\mathrm{KBr}) v$ 3444, 2922, 1648, 1598, 1220, 1178, $765 \mathrm{~cm}^{-1}$. HRMS (ESI) calcd. for $\mathrm{C}_{22} \mathrm{H}_{19} \mathrm{~N}_{2} \mathrm{O}_{4}$ $[\mathrm{M}-\mathrm{Br}]^{+}:$375.1339, found: 375.1346. 


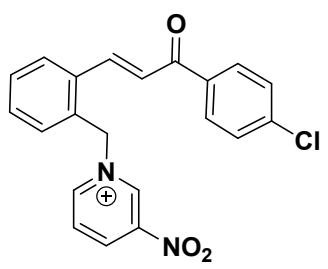

(E)-1-(2-(3-(4-chlorophenyl)-3-oxoprop-1-en-1-yl)benzyl)-3-nitropyridin-1-ium bromide (1k)

According to the general procedure, 1k was prepared on a scale of $3.0 \mathrm{mmol}$. Yellow solids, 0.92 $\mathrm{g}, 67 \%$ isolated yield obtained by filtration of the precipitate; Reaction time $=16 \mathrm{~h} ; \mathrm{m} . \mathrm{p}$. $144.9-145.4^{\circ} \mathrm{C} ;{ }^{1} \mathrm{H}$ NMR $\left(400 \mathrm{MHz}, \mathrm{DMSO}-d_{6}\right) \delta 10.24(\mathrm{~s}, 1 \mathrm{H}), 9.41-9.40\left(\mathrm{dd}, J_{1}=J_{2}=8.0 \mathrm{~Hz}\right.$, 1H), $9.37(\mathrm{~d}, J=4.0 \mathrm{~Hz}, 1 \mathrm{H}), 8.47$ (t, $J=8.0 \mathrm{~Hz}, 1 \mathrm{H}), 8.21-8.15(\mathrm{~m}, 3 \mathrm{H}), 8.09(\mathrm{~d}, J=16.0 \mathrm{~Hz}$, 1H), $7.87(\mathrm{~d}, J=16.0 \mathrm{~Hz}, 1 \mathrm{H}), 7.66(\mathrm{~d}, J=12.0 \mathrm{~Hz}, 2 \mathrm{H}), 7.59-7.50(\mathrm{~m}, 2 \mathrm{H}), 7.23(\mathrm{~d}, J=8.0 \mathrm{~Hz}$, 1H), $6.43(\mathrm{~s}, 2 \mathrm{H}) ;{ }^{13} \mathrm{C}$ NMR (100 MHz, DMSO- $\left.d_{6}\right) \delta 188.0,149.7,146.6,142.9,140.6,139.5$, $138.4,135.8,133.9,133.0,130.9,130.6,129.7,129.2,129.1,129.0,128.2,125.7,61.4 . \mathrm{IR}(\mathrm{KBr})$ $v$ 3442, 3001, 1649, 1595, 1555, $748 \mathrm{~cm}^{-1}$. HRMS (ESI) calcd. for $\mathrm{C}_{21} \mathrm{H}_{16} \mathrm{ClN}_{2} \mathrm{O}_{3}$ [M-Br] 379.0844, found: 379.0840 .

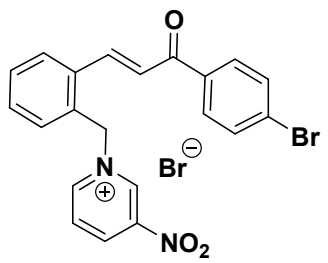

(E)-1-(2-(3-(4-bromophenyl)-3-oxoprop-1-en-1-yl)benzyl)-3-nitropyridin-1-ium bromide (11)

According to the general procedure, 11 was prepared on a scale of 3.0 mmol. Yellow solids, 1.23 $\mathrm{g}, 82 \%$ isolated yield obtained by filtration of the precipitate; Reaction time $=16 \mathrm{~h} ; \mathrm{m} . \mathrm{p}$. 176.2-177. ${ }^{\circ} \mathrm{C} ;{ }^{1} \mathrm{H}$ NMR (400 MHz, DMSO- $\left.d_{6}\right) \delta 10.24(\mathrm{~s}, 1 \mathrm{H}), 9.42-9.40(\mathrm{~m}, 1 \mathrm{H}), 9.38-9.36(\mathrm{~m}$, $1 \mathrm{H}), 8.47\left(\mathrm{dd}, J_{1}=J_{2}=8.0 \mathrm{~Hz}, 1 \mathrm{H}\right), 8.16-8.07(\mathrm{~m}, 4 \mathrm{H}), 7.86(\mathrm{~d}, J=16.0 \mathrm{~Hz}, 1 \mathrm{H}), 7.79(\mathrm{~d}, J=$ $12.0 \mathrm{~Hz}, 2 \mathrm{H}), 7.59-7.50(\mathrm{~m}, 2 \mathrm{H}), 7.24(\mathrm{~d}, J=8.0 \mathrm{~Hz}, 1 \mathrm{H}), 6.43(\mathrm{~s}, 2 \mathrm{H}) ;{ }^{13} \mathrm{C}$ NMR $(100 \mathrm{MHz}$, DMSO-d $\left.{ }_{6}\right) \delta 188.2,149.7,146.6,142.9,140.6,139.5,136.1,133.9,133.0,131.9,130.9,130.7$, $129.7,129.2,129.1,128.2,127.7,125.6,61.4 . \mathrm{IR}(\mathrm{KBr}) v 3433,2999,2938,1650,1593,1349$, 1217, $745 \mathrm{~cm}^{-1}$. HRMS (ESI) calcd. for $\mathrm{C}_{21} \mathrm{H}_{16} \mathrm{BrN}_{2} \mathrm{O}_{3}[\mathrm{M}-\mathrm{Br}]^{+}$: 423.0339, found: 423.0334 .

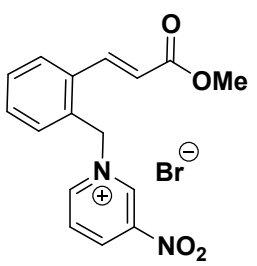

(E)-1-(2-(3-methoxy-3-oxoprop-1-en-1-yl)benzyl)-3-nitropyridin-1-ium bromide (1m) 
According to the general procedure, $1 \mathbf{m}$ was prepared on a scale of $6.0 \mathrm{mmol}$. Yellow solids, 2.09 g, $92 \%$ isolated yield obtained by filtration of the precipitate; Reaction time $=16 \mathrm{~h} ; \mathrm{m} . \mathrm{p}$. 165.0-165.9 ${ }^{\circ} \mathrm{C} ;{ }^{1} \mathrm{H}$ NMR $\left(300 \mathrm{MHz}, \mathrm{DMSO}-d_{6}\right) \delta 10.26(\mathrm{~s}, 1 \mathrm{H}), 9.41\left(\mathrm{dd}, J_{l}=J_{2}=8.0 \mathrm{~Hz}, 2 \mathrm{H}\right)$, $8.50(\mathrm{t}, J=8.0 \mathrm{~Hz}, 1 \mathrm{H}), 8.03(\mathrm{~d}, J=18.0 \mathrm{~Hz}, 1 \mathrm{H}), 7.86(\mathrm{~d}, J=12.0 \mathrm{~Hz}, 1 \mathrm{H}), 7.49(\mathrm{t}, J=8.0 \mathrm{~Hz}$, 2H), $7.25(\mathrm{t}, J=12.0 \mathrm{~Hz}, 1 \mathrm{H}), 7.57(\mathrm{~d}, J=15.0 \mathrm{~Hz}, 1 \mathrm{H}), 6.43(\mathrm{~s}, 2 \mathrm{H}), 3.74(\mathrm{~s}, 3 \mathrm{H}) ;{ }^{13} \mathrm{C}$ NMR $(75$ MHz, DMSO- $\left.d_{6}\right) \delta 166.2,149.7,146.5,142.9,140.7,140.1,133.4,132.6,130.8,129.8,129.3$, 129.1, 127.9, 121.9, 61.2, 51.8. IR (KBr) v 3432, 3028, 1718, 1548, 1221, $771 \mathrm{~cm}^{-1}$. HRMS (ESI) calcd. for $\mathrm{C}_{16} \mathrm{H}_{15} \mathrm{~N}_{2} \mathrm{O}_{4}[\mathrm{M}-\mathrm{Br}]^{+}: 299.1026$, found: 299.1025 .<smiles></smiles>

(E)-3-cyano-1-(2-(3-oxo-3-phenylprop-1-en-1-yl)benzyl)pyridin-1-ium bromide (1n)

According to the general procedure, $1 \mathbf{n}$ was prepared on a scale of $4.0 \mathrm{mmol}$. Yellow solids, 1.25 $\mathrm{g}, 78 \%$ isolated yield obtained by filtration of the precipitate; Reaction time $=16 \mathrm{~h}$; m. p. 178.6-179.4 ${ }^{\circ} \mathrm{C} ;{ }^{1} \mathrm{H}$ NMR (400 MHz, DMSO-d 6 ) $\delta 9.92(\mathrm{~s}, 1 \mathrm{H}), 9.30(\mathrm{~d}, J=8.0 \mathrm{~Hz}, 1 \mathrm{H}), 9.17(\mathrm{~d}$, $J=8.0 \mathrm{~Hz}, 1 \mathrm{H}), 8.40\left(\mathrm{dd}, J_{I}=J_{2}=8.0 \mathrm{~Hz}, 1 \mathrm{H}\right), 8.17-8.13(\mathrm{~m}, 3 \mathrm{H}), 8.04(\mathrm{~d}, J=16.0 \mathrm{~Hz}, 1 \mathrm{H}), 7.85$ (d, $J=16.0 \mathrm{~Hz}, 1 \mathrm{H}), 7.69(\mathrm{t}, J=8.0 \mathrm{~Hz}, 1 \mathrm{H}), 7.60-7.51(\mathrm{~m}, 4 \mathrm{H}), 7.33(\mathrm{~d}, J=8.0 \mathrm{~Hz}, 1 \mathrm{H}), 6.30(\mathrm{~s}$, 2H); ${ }^{13} \mathrm{C}$ NMR (75 MHz, DMSO- $\left.d_{6}\right) \delta 189.0,149.4,149.3,148.5,139.2,137.1,134.2,133.5$, $132.5,130.8,129.8,129.8,128.9,128.7,128.3,125.9,113.8,113.1,61.3$, one carbon missing in the aromatic region. IR (KBr) $v 3457,2909,1659,1590,1218,763 \mathrm{~cm}^{-1}$. HRMS (ESI) calcd. for $\mathrm{C}_{22} \mathrm{H}_{17} \mathrm{~N}_{2} \mathrm{O}[\mathrm{M}-\mathrm{Br}]^{+}: 325.1335$, found: 325.1338 .

\section{Experimental data for bi-bridged benzoazepines 3}

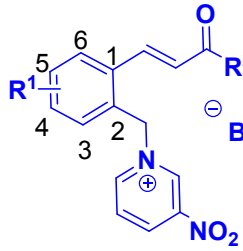

1

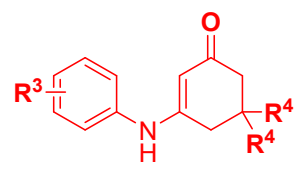

2

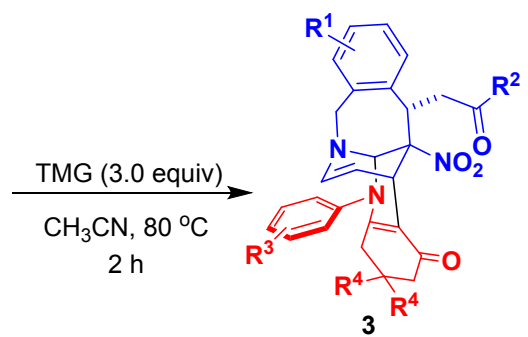

$3^{\mathrm{R}}$

General procedure: To a $5.0 \mathrm{~mL}$ vial were successively added chalcone-based pyridinium salts $1(0.15 \mathrm{mmol})$, enaminones $2(0.375 \mathrm{mmol})$, TMG $(0.45 \mathrm{mmol})$ and $1.0 \mathrm{~mL} \mathrm{CH} \mathrm{CHN}_{3}$. The resulting mixture was stirred at $80^{\circ} \mathrm{C}$ in oil bath for $2 \mathrm{~h}$, and then the reaction mixture was directly 
subjected to flash column chromatography on silica gel (petroleum ether/ ethyl acetate) to afford the corresponding bi-bridged benzoazepines 3 .

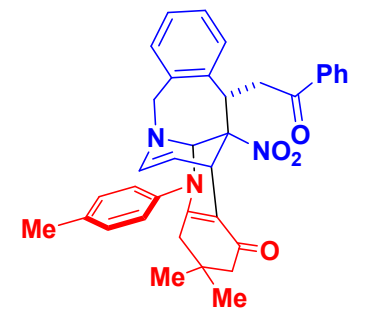

3,3-dimethyl-12a-nitro-12-(2-oxo-2-phenylethyl)-5-( $p$-tolyl)-3,4,5,5a,7,12,12a,13-octahydro-6,13ethenobenzo[5,6]azepino[2,3-b]quinolin-1(2H)-one (3a)

Yellow solid obtained by column chromatography (petroleum ether/ethyl acetate $=3: 1$ ); $65.6 \mathrm{mg}$, $76 \%$ yield; $\mathrm{dr}>20: 1 ;$ reaction time $=2 \mathrm{~h} ; \mathrm{mp} 208.8-209.7^{\circ} \mathrm{C} ;{ }^{1} \mathrm{H}$ NMR $\left(400 \mathrm{MHz}, \mathrm{CDCl}_{3}\right), \delta 7.96$ $(\mathrm{d}, J=8.0 \mathrm{~Hz}, 2 \mathrm{H}), 7.58(\mathrm{t}, J=8.0 \mathrm{~Hz}, 1 \mathrm{H}), 7.46(\mathrm{t}, J=8.0 \mathrm{~Hz}, 2 \mathrm{H}), 7.17-7.05(\mathrm{~m}, 4 \mathrm{H}), 6.92(\mathrm{dd}$, $\left.J_{l}=J_{2}=8.0 \mathrm{~Hz}, 4 \mathrm{H}\right), 6.16(\mathrm{~d}, J=8.0 \mathrm{~Hz}, 1 \mathrm{H}), 5.23(\mathrm{t}, J=8.0 \mathrm{~Hz}, 1 \mathrm{H}), 5.06(\mathrm{~s}, 1 \mathrm{H}), 4.53(\mathrm{~d}, J=$ $4.0 \mathrm{~Hz}, 1 \mathrm{H}), 4.44-4.31(\mathrm{~m}, 3 \mathrm{H}), 3.72-3.58(\mathrm{~m}, 2 \mathrm{H}), 2.36(\mathrm{~s}, 3 \mathrm{H}), 2.14(\mathrm{~d}, J=4.0 \mathrm{~Hz}, 2 \mathrm{H}), 1.91(\mathrm{~d}$, $J=20.0 \mathrm{~Hz}, 1 \mathrm{H}), 1.72(\mathrm{~d}, J=20.0 \mathrm{~Hz}, 1 \mathrm{H}), 0.87(\mathrm{~s}, 3 \mathrm{H}), 0.81(\mathrm{~s}, 3 \mathrm{H}) ;{ }^{13} \mathrm{C}$ NMR $(100 \mathrm{MHz}$, $\left.\mathrm{CDCl}_{3}\right) \delta 196.2,192.4,156.4,139.2,138.0,137.5,136.3,135.1,133.7,133.5,129.9,128.7,128.1$, 128.0, 127.6, 127.4, 125.8, 108.0, 107.1, 88.9, 74.0, 58.5, 49.3, 40.7, 38.5, 35.3, 32.7, 29.3, 28.5, 27.5, 21.1. IR (KBr) $v$ 3421, 2950, 1627, 1578, 1391, $757 \mathrm{~cm}^{-1}$. HRMS (ESI) calcd for $\mathrm{C}_{36} \mathrm{H}_{36} \mathrm{~N}_{3} \mathrm{O}_{4}[\mathrm{M}+\mathrm{H}]^{+}$574.2700, found 574.2698.

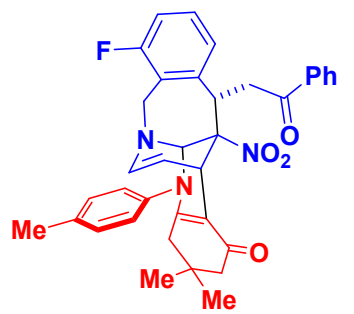

8-fluoro-3,3-dimethyl-12a-nitro-12-(2-oxo-2-phenylethyl)-5-( $p$-tolyl)-3,4,5,5a,7,12,12a,13-octahy dro-6,13-ethenobenzo[5,6]azepino[2,3-b]quinolin-1(2H)-one (3b)

Yellow solid obtained by column chromatography (petroleum ether/ethyl acetate $=3: 1$ ); $40.7 \mathrm{mg}$, $46 \%$ yield; $\mathrm{dr}>20: 1 ;$ reaction time $=2 \mathrm{~h} ; \mathrm{mp} 228.6-229.3^{\circ} \mathrm{C} ;{ }^{1} \mathrm{H}$ NMR $\left(400 \mathrm{MHz}, \mathrm{CDCl}_{3}\right), \delta 7.93$ $(\mathrm{d}, J=8.0 \mathrm{~Hz}, 2 \mathrm{H}), 7.57(\mathrm{t}, J=8.0 \mathrm{~Hz}, 1 \mathrm{H}), 7.45(\mathrm{t}, J=8.0 \mathrm{~Hz}, 2 \mathrm{H}), 7.17(\mathrm{~d}, J=4.0 \mathrm{~Hz}, 2 \mathrm{H})$, 7.01-6.85 (m, 4H), $6.68(\mathrm{~d}, J=8.0 \mathrm{~Hz}, 1 \mathrm{H}), 6.17(\mathrm{~d}, J=4.0 \mathrm{~Hz}, 1 \mathrm{H}), 5.21(\mathrm{t}, J=8.0 \mathrm{~Hz}, 1 \mathrm{H}), 5.08$ (s, 1H), $4.69(\mathrm{~d}, J=16.0 \mathrm{~Hz}, 1 \mathrm{H}), 4.53(\mathrm{~d}, J=4.0 \mathrm{~Hz}, 1 \mathrm{H}), 4.42\left(\mathrm{dd}, J_{l}=J_{2}=4.0 \mathrm{~Hz}, 1 \mathrm{H}\right), 4.15$ 
$(\mathrm{d}, J=16.0 \mathrm{~Hz}, 1 \mathrm{H}), 3.69-3.55(\mathrm{~m}, 2 \mathrm{H}), 2.35(\mathrm{~s}, 3 \mathrm{H}), 2.14(\mathrm{t}, J=16.0 \mathrm{~Hz}, 2 \mathrm{H}), 1.91(\mathrm{~d}, J=16.0$ $\mathrm{Hz}, 1 \mathrm{H}), 1.75(\mathrm{~d}, J=16.0 \mathrm{~Hz}, 1 \mathrm{H}), 0.86(\mathrm{~s}, 3 \mathrm{H}), 0.82(\mathrm{~s}, 3 \mathrm{H}) ;{ }^{13} \mathrm{C} \mathrm{NMR}\left(100 \mathrm{MHz}, \mathrm{CDCl}_{3}\right) \delta$ $195.8,192.2,161.2,158.7,156.0,139.1,138.1,137.6,136.1,133.6,133.5,128.6,128.1\left(\mathrm{~d},{ }^{3} J_{\mathrm{C}-\mathrm{F}}=\right.$ $9.0 \mathrm{~Hz}, 1 \mathrm{C}), 127.9,125.4\left(\mathrm{~d},{ }^{3} J_{\mathrm{C}-\mathrm{F}}=13.0 \mathrm{~Hz}, 1 \mathrm{C}\right), 121.7,114.6\left(\mathrm{~d},{ }^{2} J_{\mathrm{C}-\mathrm{F}}=23.0 \mathrm{~Hz}, 1 \mathrm{C}\right), 107.5$, 107.2, 88.7, 73.8, 50.4, 49.3, 40.6, 38.5, 35.7, 32.6, 29.3, 28.4, 27.5, 21.0. IR (KBr) v 3449, 2956, 1624, 1572, 1397, 1240, 758cm $\mathrm{cm}^{-1}$. HRMS (ESI) calcd for $\mathrm{C}_{36} \mathrm{H}_{35} \mathrm{FN}_{3} \mathrm{O}_{4}[\mathrm{M}+\mathrm{H}]^{+} 592.2606$, found 592.2619 .

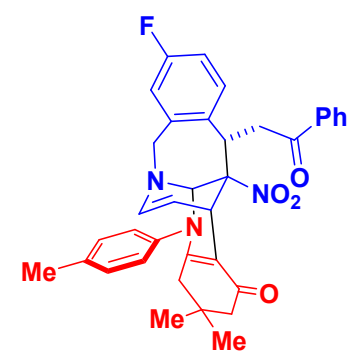

9-fluoro-3,3-dimethyl-12a-nitro-12-(2-oxo-2-phenylethyl)-5-(p-tolyl)-3,4,5,5a,7,12,12a,13-octahy dro-6,13-ethenobenzo[5,6]azepino[2,3-b]quinolin-1(2H)-one (3c)

Yellow solid obtained by column chromatography (petroleum ether/ethyl acetate $=3: 1$ ); $48.6 \mathrm{mg}$, $55 \%$ yield; $\mathrm{dr}>20: 1 ;$ reaction time $=2 \mathrm{~h} ; \mathrm{mp} 197.5-198.3^{\circ} \mathrm{C} ;{ }^{1} \mathrm{H}$ NMR $\left(400 \mathrm{MHz}, \mathrm{CDCl}_{3}\right), \delta 7.94$ (d, $J=8.0 \mathrm{~Hz}, 2 \mathrm{H}), 7.58$ (t, $J=8.0 \mathrm{~Hz}, 1 \mathrm{H}), 7.46$ (t, $J=8.0 \mathrm{~Hz}, 2 \mathrm{H}), 7.17(\mathrm{~d}, J=8.0 \mathrm{~Hz}, 2 \mathrm{H})$, 6.99-6.82 (m, 3H), 6.77-6.68 (m, 2H), $6.15(\mathrm{~d}, J=8.0 \mathrm{~Hz}, 1 \mathrm{H}), 5.23(\mathrm{t}, J=8.0 \mathrm{~Hz}, 1 \mathrm{H}), 5.02(\mathrm{~s}$, 1H), $4.53(\mathrm{~d}, J=4.0 \mathrm{~Hz}, 1 \mathrm{H}), 4.40(\mathrm{~d}, J=16.0 \mathrm{~Hz}, 1 \mathrm{H}), 4.30$ (q, $J=8.0 \mathrm{~Hz}, 2 \mathrm{H}), 3.61$ (d, $J=8.0$ Hz, 2H), 2.36 (s, 3H), 2.14 (t, $J=16.0 \mathrm{~Hz}, 2 \mathrm{H}), 1.91$ (d, $J=16.0 \mathrm{~Hz}, 1 \mathrm{H}), 1.73$ (d, $J=16.0 \mathrm{~Hz}$, 1H), 0.86 (s, 3H), 0.81 (s, 3H); ${ }^{13} \mathrm{C}$ NMR (100 MHz, $\left.\mathrm{CDCl}_{3}\right) \delta$ 196.0, 192.3, 163.0, 160.5, 156.2, $139.8\left(\mathrm{~d},{ }^{3} J_{\mathrm{C}-\mathrm{F}}=6.0 \mathrm{~Hz}, 1 \mathrm{C}\right), 139.1,138.1,136.2,133.6,133.5,130.9,128.7,128.0,127.4\left(\mathrm{~d},{ }^{3} J_{\mathrm{C}-\mathrm{F}}\right.$ $=8.0 \mathrm{~Hz}, 1 \mathrm{C}), 115.1\left(\mathrm{~d},{ }^{2} J_{\mathrm{C}-\mathrm{F}}=21.0 \mathrm{~Hz}, 1 \mathrm{C}\right), 114.9\left(\mathrm{~d},{ }^{2} J_{\mathrm{C}-\mathrm{F}}=20.0 \mathrm{~Hz}, 1 \mathrm{C}\right), 108.2,107.0,88.8$, 74.0, 58.2, 49.3, 40.6, 38.0, 35.4, 32.6, 29.2, 28.5, 27.5, 21.1. IR (KBr) v 3444, 2936, 1626, 1580 , 1390, $736 \mathrm{~cm}^{-1}$. HRMS (ESI) calcd for $\mathrm{C}_{36} \mathrm{H}_{35} \mathrm{FN}_{3} \mathrm{O}_{4}[\mathrm{M}+\mathrm{H}]^{+}$592.2606, found 592.2620.

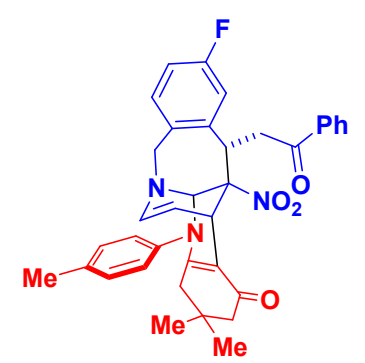


10-fluoro-3,3-dimethyl-12a-nitro-12-(2-oxo-2-phenylethyl)-5-( $p$-tolyl)-3,4,5,5a,7,12,12a,13-octah ydro-6,13-ethenobenzo[5,6]azepino[2,3-b]quinolin-1(2H)-one (3d)

Yellow solid obtained by column chromatography (petroleum ether/ethyl acetate $=3: 1$ ); $51.3 \mathrm{mg}$, $58 \%$ yield; $\mathrm{dr}>20: 1 ;$ reaction time $=2 \mathrm{~h} ; \mathrm{mp} 216.9-217.1^{\circ} \mathrm{C} ;{ }^{1} \mathrm{H}$ NMR $\left(400 \mathrm{MHz}, \mathrm{CDCl}_{3}\right), \delta 7.96$ $(\mathrm{d}, J=8.0 \mathrm{~Hz}, 2 \mathrm{H}), 7.60(\mathrm{t}, J=8.0 \mathrm{~Hz}, 1 \mathrm{H}), 7.48(\mathrm{t}, J=8.0 \mathrm{~Hz}, 2 \mathrm{H}), 7.17(\mathrm{~d}, J=4.0 \mathrm{~Hz}, 2 \mathrm{H})$, 6.96-6.82 (m, 4H), 6.60 (d, $J=8.0 \mathrm{~Hz}, 1 \mathrm{H}), 6.15(\mathrm{~d}, J=8.0 \mathrm{~Hz}, 1 \mathrm{H}), 5.23(\mathrm{t}, J=8.0 \mathrm{~Hz}, 1 \mathrm{H}), 5.04$ (s, 1H), $4.52(\mathrm{~d}, J=8.0 \mathrm{~Hz}, 1 \mathrm{H}), 4.36-4.31(\mathrm{~m}, 3 \mathrm{H}), 3.67-3.52(\mathrm{~m}, 2 \mathrm{H}), 2.37(\mathrm{~s}, 3 \mathrm{H}), 2.14(\mathrm{t}, J=$ $16.0 \mathrm{~Hz}, 2 \mathrm{H}), 1.91(\mathrm{~d}, J=16.0 \mathrm{~Hz}, 1 \mathrm{H}), 1.72(\mathrm{~d}, J=16.0 \mathrm{~Hz}, 1 \mathrm{H}), 0.87(\mathrm{~s}, 3 \mathrm{H}), 0.82(\mathrm{~s}, 3 \mathrm{H}) ;{ }^{13} \mathrm{C}$ $\operatorname{NMR}\left(100 \mathrm{MHz}, \mathrm{CDCl}_{3}\right) \delta 195.9,192.3,163.3,160.8,156.2,139.2,138.1,137.9\left(\mathrm{~d},{ }^{3} J_{\mathrm{C}-\mathrm{F}}=7.0\right.$ $\mathrm{Hz}, 1 \mathrm{C}), 136.1,133.7,133.1\left(\mathrm{~d},{ }^{4} J_{\mathrm{C}-\mathrm{F}}=3.0 \mathrm{~Hz}, 1 \mathrm{C}\right), 129.4\left(\mathrm{~d},{ }^{3} J_{\mathrm{C}-\mathrm{F}}=8.0 \mathrm{~Hz}, 1 \mathrm{C}\right), 128.8,128.0$, $127.5,114.4\left(\mathrm{~d},{ }^{2} J_{\mathrm{C}-\mathrm{F}}=21.0 \mathrm{~Hz}, 1 \mathrm{C}\right), 113.4\left(\mathrm{~d},{ }^{2} J_{\mathrm{C}-\mathrm{F}}=23.0 \mathrm{~Hz}, 1 \mathrm{C}\right), 108.1,107.0,88.6,74.0,57.9$, 49.4, 40.7, 38.4, 35.4, 32.7, 29.3, 28.6, 27.5, 21.1. IR (KBr) v 3387, 2975, 2892, 1639, 1387, $1088,1049,881 \mathrm{~cm}^{-1}$. HRMS (ESI) calcd for $\mathrm{C}_{36} \mathrm{H}_{35} \mathrm{FN}_{3} \mathrm{O}_{4}[\mathrm{M}+\mathrm{H}]^{+}$592.2606, found 592.2594.

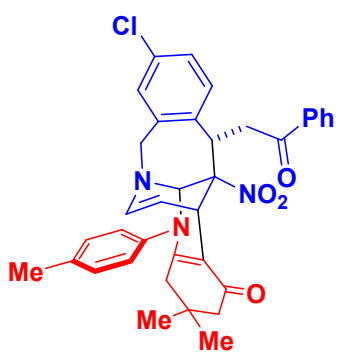

9-chloro-3,3-dimethyl-12a-nitro-12-(2-oxo-2-phenylethyl)-5-( $p$-tolyl)-3,4,5,5a, 7,12,12a,13-octahy dro-6,13-ethenobenzo[5,6]azepino[2,3-b]quinolin-1(2H)-one (3e)

Yellow solid obtained by column chromatography (petroleum ether/ethyl acetate $=3: 1$ ); $62.4 \mathrm{mg}$, $68 \%$ yield; $\mathrm{dr}>20: 1 ;$ reaction time $=2 \mathrm{~h} ; \mathrm{mp} 230.6-231.2^{\circ} \mathrm{C} ;{ }^{1} \mathrm{H}$ NMR $\left(400 \mathrm{MHz}, \mathrm{CDCl}_{3}\right), \delta 7.95$ $(\mathrm{d}, J=8.0 \mathrm{~Hz}, 2 \mathrm{H}), 7.60$ (t, $J=8.0 \mathrm{~Hz}, 1 \mathrm{H}), 7.48(\mathrm{t}, J=8.0 \mathrm{~Hz}, 2 \mathrm{H}), 7.19(\mathrm{~d}, J=8.0 \mathrm{~Hz}, 2 \mathrm{H})$, $7.05\left(\mathrm{dd}, J_{I}=J_{2}=4.0 \mathrm{~Hz}, 1 \mathrm{H}\right), 6.98-6.80(\mathrm{~m}, 4 \mathrm{H}), 6.15(\mathrm{~d}, J=8.0 \mathrm{~Hz}, 1 \mathrm{H}), 5.24(\mathrm{t}, J=8.0 \mathrm{~Hz}$, 1H), $5.02(\mathrm{~s}, 1 \mathrm{H}), 4.52(\mathrm{~d}, J=4.0 \mathrm{~Hz}, 1 \mathrm{H}), 4.43-4.28(\mathrm{~m}, 3 \mathrm{H}), 3.62(\mathrm{~d}, J=4.0 \mathrm{~Hz}, 2 \mathrm{H}), 2.39$ (s, 3H), 2.15 (t, $J=16.0 \mathrm{~Hz}, 2 \mathrm{H}), 1.91$ (d, $J=16.0 \mathrm{~Hz}, 1 \mathrm{H}), 1.74$ (d, $J=16.0 \mathrm{~Hz}, 1 \mathrm{H}), 0.87$ (s, 3H), $0.83(\mathrm{~s}, 3 \mathrm{H}) ;{ }^{13} \mathrm{C}$ NMR $\left(100 \mathrm{MHz}, \mathrm{CDCl}_{3}\right) \delta 196.0,192.4,156.2,139.5,139.2,138.2,136.2$, 133.9, 133.7, 133.6, 133.4, 130.8, 129.9, 128.8, 128.1, 127.4, 127.2, 108.3, 107.0, 88.7, 74.0, 58.1, 49.4, 40.7, 38.2, 35.4, 32.7, 29.3, 28.6, 27.6, 21.2. IR (KBr) v 3429, 2953, 1688, 1628, 1580 , 1391, 1138, 759 $\mathrm{cm}^{-1}$. HRMS (ESI) calcd for $\mathrm{C}_{36} \mathrm{H}_{35} \mathrm{ClN}_{3} \mathrm{O}_{4}[\mathrm{M}+\mathrm{H}]^{+} 608.2311$, found 608.2322. 


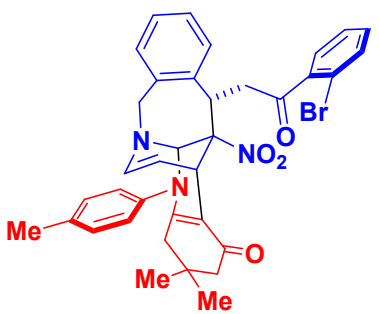

12-(2-(2-bromophenyl)-2-oxoethyl)-3,3-dimethyl-12a-nitro-5-( $p$-tolyl)-3,4,5,5a,7,12,12a,13-octah ydro-6,13-ethenobenzo[5,6]azepino[2,3-b]quinolin-1(2H)-one (3f)

Yellow solid obtained by column chromatography (petroleum ether/ethyl acetate $=3: 1$ ); $52.6 \mathrm{mg}$, $54 \%$ yield; $\mathrm{dr}>20: 1 ;$ reaction time $=2 \mathrm{~h} ; \mathrm{mp} 214.6-215.5^{\circ} \mathrm{C} ;{ }^{1} \mathrm{H}$ NMR $\left(400 \mathrm{MHz}, \mathrm{CDCl}_{3}\right), \delta 7.59$ $(\mathrm{d}, J=8.0 \mathrm{~Hz}, 1 \mathrm{H}), 7.35-7.27(\mathrm{~m}, 3 \mathrm{H}), 7.17-7.12(\mathrm{~m}, 4 \mathrm{H}), 6.97\left(\mathrm{dd}, J_{I}=J_{2}=8.0 \mathrm{~Hz}, 4 \mathrm{H}\right), 6.11(\mathrm{~d}$, $J=8.0 \mathrm{~Hz}, 1 \mathrm{H}), 5.25(\mathrm{t}, J=8.0 \mathrm{~Hz}, 1 \mathrm{H}), 5.01(\mathrm{~s}, 1 \mathrm{H}), 4.54(\mathrm{~d}, J=4.0 \mathrm{~Hz}, 1 \mathrm{H}), 4.29(\mathrm{~s}, 2 \mathrm{H}), 4.24$ $\left(\mathrm{dd}, J_{I}=J_{2}=4.0 \mathrm{~Hz}, 1 \mathrm{H}\right), 3.71(\mathrm{~d}, J=16.0 \mathrm{~Hz}, 1 \mathrm{H}), 3.45\left(\mathrm{dd}, J_{l}=J_{2}=12.0 \mathrm{~Hz}, 1 \mathrm{H}\right), 2.35(\mathrm{~s}, 3 \mathrm{H})$, $2.13(\mathrm{t}, J=16.0 \mathrm{~Hz}, 2 \mathrm{H}), 1.90(\mathrm{~d}, J=16.0 \mathrm{~Hz}, 1 \mathrm{H}), 1.70(\mathrm{~d}, J=16.0 \mathrm{~Hz}, 1 \mathrm{H}), 0.86(\mathrm{~s}, 3 \mathrm{H}), 0.79$ (s, 3H); ${ }^{13} \mathrm{C}$ NMR $\left(100 \mathrm{MHz}, \mathrm{CDCl}_{3}\right) \delta 199.9,192.2,156.2,140.4,139.2,138.0,137.6,134.7$, $133.9,133.6,132.0,129.8,128.9,128.0,127.8,127.4,127.3,126.5,118.8,108.1,107.1,88.6$, 73.9, 58.5, 49.4, 40.7, 39.5, 39.2, 32.7, 29.2, 28.6, 27.5, 21.1. IR (KBr) v 3424, 2955, 2871, 1624 , 1578, 1393, $732 \mathrm{~cm}^{-1}$. HRMS (ESI) calcd for $\mathrm{C}_{36} \mathrm{H}_{35} \mathrm{BrN}_{3} \mathrm{O}_{4}[\mathrm{M}+\mathrm{H}]^{+} 652.1805$, found 652.1796 .

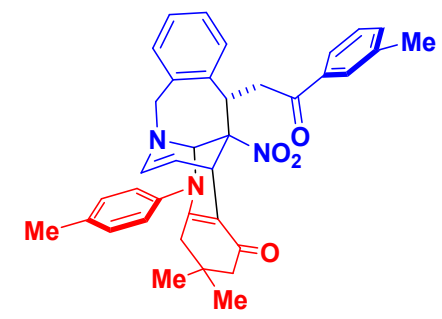

3,3-dimethyl-12a-nitro-12-(2-oxo-2-( $m$-tolyl)ethyl)-5-(p-tolyl)-3,4,5,5a,7,12,12a,13-octahydro-6,1 3-ethenobenzo[5,6]azepino[2,3-b]quinolin-1(2H)-one (3g)

Yellow solid obtained by column chromatography (petroleum ether/ethyl acetate $=3: 1$ ); $44.5 \mathrm{mg}$, $51 \%$ yield; $\mathrm{dr}>20: 1 ;$ reaction time $=2 \mathrm{~h} ; \mathrm{mp} 232.4-233.2^{\circ} \mathrm{C} ;{ }^{1} \mathrm{H}$ NMR $\left(400 \mathrm{MHz}, \mathrm{CDCl}_{3}\right), \delta 7.76$ $(\mathrm{d}, J=8.0 \mathrm{~Hz}, 2 \mathrm{H}), 7.40-7.32(\mathrm{~m}, 2 \mathrm{H}), 7.14$ (t, $J=8.0 \mathrm{~Hz}, 2 \mathrm{H}), 7.10$ (d, $J=4.0 \mathrm{~Hz}, 1 \mathrm{H}), 7.05(\mathrm{t}, J$ $=8.0 \mathrm{~Hz}, 1 \mathrm{H}), 6.92\left(\mathrm{dd}, J_{I}=J_{2}=8.0 \mathrm{~Hz}, 4 \mathrm{H}\right), 6.15(\mathrm{~d}, J=4.0 \mathrm{~Hz}, 1 \mathrm{H}), 5.23(\mathrm{t}, J=8.0 \mathrm{~Hz}, 1 \mathrm{H})$, $5.06(\mathrm{~s}, 1 \mathrm{H}), 4.54(\mathrm{~d}, J=4.0 \mathrm{~Hz}, 1 \mathrm{H}), 4.43-4.31(\mathrm{~m}, 3 \mathrm{H}), 3.71-3.57(\mathrm{~m}, 2 \mathrm{H}), 2.40(\mathrm{~s}, 3 \mathrm{H}), 2.36(\mathrm{~s}$, 3H), $2.15(\mathrm{t}, J=16.0 \mathrm{~Hz}, 2 \mathrm{H}), 1.92(\mathrm{~d}, J=16.0 \mathrm{~Hz}, 1 \mathrm{H}), 1.73(\mathrm{~d}, J=16.0 \mathrm{~Hz}, 1 \mathrm{H}), 0.87(\mathrm{~s}, 3 \mathrm{H})$, 0.82 (s, 3H); ${ }^{13} \mathrm{C}$ NMR (100 MHz, $\left.\mathrm{CDCl}_{3}\right) \delta 196.2,192.2,156.3,139.2,138.4,138.0,137.4$, $136.3,135.1,134.2,133.7,130.5,129.8,128.5,128.0,127.6,127.3,125.7,125.2,108.0,107.1$, 
$88.8,74.0,58.5,49.3,40.7,38.5,35.3,32.6,29.2,28.5,27.5,21.2,21.1 . \mathrm{IR}(\mathrm{KBr}) v 3467,2954$, $1684,1622,1572,1394,1138,731 \mathrm{~cm}^{-1}$. HRMS (ESI) calcd for $\mathrm{C}_{37} \mathrm{H}_{38} \mathrm{~N}_{3} \mathrm{O}_{4}[\mathrm{M}+\mathrm{H}]^{+}$588.2857, found 588.2854 .

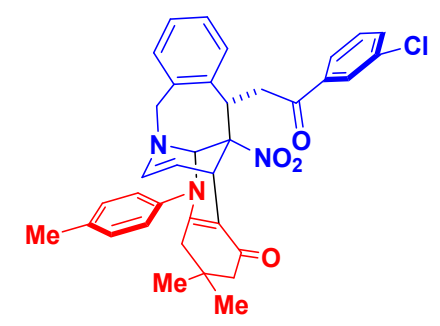

12-(2-(3-chlorophenyl)-2-oxoethyl)-3,3-dimethyl-12a-nitro-5-( $p$-tolyl)-3,4,5,5a,7,12,12a,13-octah ydro-6,13-ethenobenzo[5,6]azepino[2,3-b]quinolin-1(2H)-one (3h)

Yellow solid obtained by column chromatography (petroleum ether/ethyl acetate $=3: 1$ ); $40.2 \mathrm{mg}$, $44 \%$ yield; $\mathrm{dr}>20: 1$; reaction time $=2 \mathrm{~h} ; \mathrm{mp} 223.7-224.5^{\circ} \mathrm{C} ;{ }^{1} \mathrm{H}$ NMR $\left(400 \mathrm{MHz}, \mathrm{CDCl}_{3}\right), \delta 7.89$ (s, 1H), $7.84(\mathrm{~d}, J=8.0 \mathrm{~Hz}, 1 \mathrm{H}), 7.53(\mathrm{~d}, J=4.0 \mathrm{~Hz}, 1 \mathrm{H}), 7.40(\mathrm{~d}, J=8.0 \mathrm{~Hz}, 1 \mathrm{H}), 7.14-7.05(\mathrm{~m}$, $4 \mathrm{H}), 6.91\left(\mathrm{dd}, J_{l}=J_{2}=8.0 \mathrm{~Hz}, 4 \mathrm{H}\right), 6.15(\mathrm{~d}, J=4.0 \mathrm{~Hz}, 1 \mathrm{H}), 5.22(\mathrm{t}, J=8.0 \mathrm{~Hz}, 1 \mathrm{H}), 5.04(\mathrm{~s}$, 1H), 4.53 (d, $J=4.0 \mathrm{~Hz}, 1 \mathrm{H}), 4.42-4.31(\mathrm{~m}, 3 \mathrm{H}), 3.60$ (d, $J=8.0 \mathrm{~Hz}, 2 \mathrm{H}), 2.35$ (s, 3H), 2.13 (t, $J$ $=16.0 \mathrm{~Hz}, 2 \mathrm{H}), 1.91(\mathrm{~d}, J=16.0 \mathrm{~Hz}, 1 \mathrm{H}), 1.72(\mathrm{~d}, J=16.0 \mathrm{~Hz}, 1 \mathrm{H}), 0.86(\mathrm{~s}, 3 \mathrm{H}), 0.80(\mathrm{~s}, 3 \mathrm{H})$; ${ }^{13} \mathrm{C}$ NMR $\left(100 \mathrm{MHz}, \mathrm{CDCl}_{3}\right) \delta 194.9,192.2,156.3,139.1,138.0,137.8,137.5,135.0,134.8$, 133.7, 133.4, 130.4, 130.0, 128.1, 128.0, 127.7, 127.4, 126.1, 125.6, 107.9, 107.0, 88.8, 73.9, 58.4, 49.3, 40.6, 38.4, 35.5, 32.6, 29.3, 28.5, 27.5, 21.0. IR (KBr) v 3464, 2955, 1622, 1566, 1397, 738 $\mathrm{cm}^{-1}$. HRMS (ESI) calcd for $\mathrm{C}_{36} \mathrm{H}_{35} \mathrm{ClN}_{3} \mathrm{O}_{4}[\mathrm{M}+\mathrm{H}]^{+}$608.2311, found 608.2302.

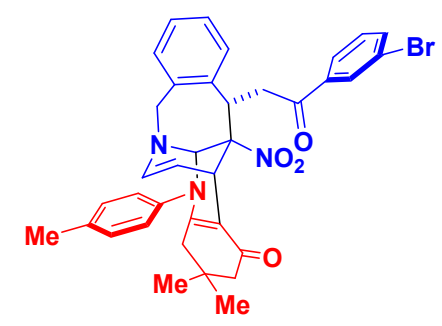

12-(2-(3-bromophenyl)-2-oxoethyl)-3,3-dimethyl-12a-nitro-5-( $p$-tolyl)-3,4,5,5a,7,12,12a,13-octah ydro-6,13-ethenobenzo[5,6]azepino[2,3-b]quinolin-1(2H)-one (3i)

Yellow solid obtained by column chromatography (petroleum ether/ethyl acetate $=3: 1$ ); $45.9 \mathrm{mg}$, $47 \%$ yield; $\mathrm{dr}>20: 1 ;$ reaction time $=2 \mathrm{~h} ; \mathrm{mp} 223.3-224.1^{\circ} \mathrm{C} ;{ }^{1} \mathrm{H}$ NMR $\left(400 \mathrm{MHz}, \mathrm{CDCl}_{3}\right), \delta 8.05$ (s, 1H), 7.89 (d, $J=8.0 \mathrm{~Hz}, 1 \mathrm{H}), 7.70(\mathrm{~d}, J=8.0 \mathrm{~Hz}, 1 \mathrm{H}), 7.37-7.32(\mathrm{~m}, 1 \mathrm{H}), 7.15-7.08$ (m, 4H), $6.92\left(\mathrm{dd}, J_{l}=J_{2}=8.0 \mathrm{~Hz}, 4 \mathrm{H}\right), 6.15(\mathrm{~d}, J=4.0 \mathrm{~Hz}, 1 \mathrm{H}), 5.22(\mathrm{t}, J=8.0 \mathrm{~Hz}, 1 \mathrm{H}), 5.05(\mathrm{~s}, 1 \mathrm{H})$, $4.52(\mathrm{~d}, J=8.0 \mathrm{~Hz}, 1 \mathrm{H}), 4.43-4.31(\mathrm{~m}, 3 \mathrm{H}), 3.61(\mathrm{~d}, J=8.0 \mathrm{~Hz}, 2 \mathrm{H}), 2.36(\mathrm{~s}, 3 \mathrm{H}), 2.17$ (t, $J=16.0$ 
$\mathrm{Hz}, 2 \mathrm{H}), 1.91(\mathrm{~d}, J=16.0 \mathrm{~Hz}, 1 \mathrm{H}), 1.72(\mathrm{~d}, J=16.0 \mathrm{~Hz}, 1 \mathrm{H}), 0.86(\mathrm{~s}, 3 \mathrm{H}), 0.81(\mathrm{~s}, 3 \mathrm{H}) ;{ }^{13} \mathrm{C}$ NMR $\left(100 \mathrm{MHz}, \mathrm{CDCl}_{3}\right) \delta 194.8,192.3,156.3,139.2,138.0,137.9,137.5,136.3,134.8,133.7,131.0$, $130.3,129.8,128.1,127.7,127.4,126.5,125.6,123.1,107.9,107.0,88.8,73.9,58.5,49.3,40.7$, 38.4, 35.5, 32.6, 29.3, 28.5, 27.5, 21.1. IR (KBr) v 3465, 2955, 1691, 1621, 1565, 1397, $741 \mathrm{~cm}^{-1}$. HRMS (ESI) calcd for $\mathrm{C}_{36} \mathrm{H}_{35} \mathrm{BrN}_{3} \mathrm{O}_{4}[\mathrm{M}+\mathrm{H}]^{+}$652.1805, found 652.1791 .

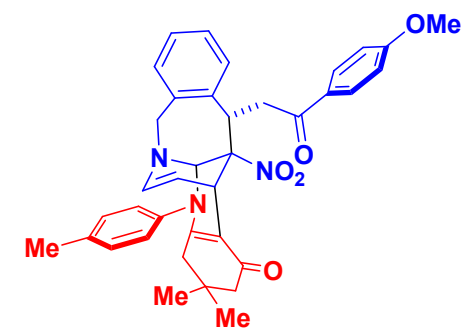

12-(2-(4-methoxyphenyl)-2-oxoethyl)-3,3-dimethyl-12a-nitro-5-( $p$-tolyl)-3,4,5,5a,7,12,12a,13-oct ahydro-6,13-ethenobenzo[5,6]azepino[2,3-b]quinolin-1(2H)-one (3j)

Yellow solid obtained by column chromatography (petroleum ether/ethyl acetate $=3: 1$ ); $81.0 \mathrm{mg}$, $89 \%$ yield; $\mathrm{dr}>20: 1 ;$ reaction time $=2 \mathrm{~h} ; \mathrm{mp} 237.7-238.0^{\circ} \mathrm{C} ;{ }^{1} \mathrm{H}$ NMR $\left(400 \mathrm{MHz}, \mathrm{CDCl}_{3}\right), \delta 7.93$ $(\mathrm{d}, J=8.0 \mathrm{~Hz}, 2 \mathrm{H}), 7.14(\mathrm{t}, J=8.0 \mathrm{~Hz}, 2 \mathrm{H}), 7.09(\mathrm{~d}, J=8.0 \mathrm{~Hz}, 1 \mathrm{H}), 7.04(\mathrm{t}, J=8.0 \mathrm{~Hz}, 1 \mathrm{H})$, 6.99-6.88 (m, 6H), $6.14(\mathrm{~d}, J=4.0 \mathrm{~Hz}, 1 \mathrm{H}), 5.22(\mathrm{t}, J=8.0 \mathrm{~Hz}, 1 \mathrm{H}), 5.05(\mathrm{~s}, 1 \mathrm{H}), 4.52\left(\mathrm{dd}, J_{l}=\right.$ $\left.J_{2}=4.0 \mathrm{~Hz}, 1 \mathrm{H}\right), 4.42-4.30(\mathrm{~m}, 3 \mathrm{H}), 3.84(\mathrm{~s}, 3 \mathrm{H}), 3.66-3.51(\mathrm{~m}, 2 \mathrm{H}), 2.35(\mathrm{~s}, 3 \mathrm{H}), 2.13(\mathrm{t}, J=16.0$ $\mathrm{Hz}, 2 \mathrm{H}), 1.91(\mathrm{~d}, J=16.0 \mathrm{~Hz}, 1 \mathrm{H}), 1.72(\mathrm{~d}, J=16.0 \mathrm{~Hz}, 1 \mathrm{H}), 0.86(\mathrm{~s}, 3 \mathrm{H}), 0.80(\mathrm{~s}, 3 \mathrm{H}) ;{ }^{13} \mathrm{C} \mathrm{NMR}$ $\left(100 \mathrm{MHz}, \mathrm{CDCl}_{3}\right) \delta 194.5,192.2,163.7,156.2,139.2,137.9,137.4,135.2,133.7,130.3,129.4$, $128.0,127.5,127.3,126.8,125.7,113.7,108.0,107.1,88.9,74.0,58.5,55.4,49.3,40.6,38.5$, 34.8, 32.6, 29.2, 28.5, 27.5, 21.0. IR (KBr) v 3438, 2952, 1585, 1391, 1261, 1176, $838 \mathrm{~cm}^{-1}$. HRMS (ESI) calcd for $\mathrm{C}_{37} \mathrm{H}_{38} \mathrm{~N}_{3} \mathrm{O}_{5}[\mathrm{M}+\mathrm{H}]^{+} 604.2806$, found 604.2817.

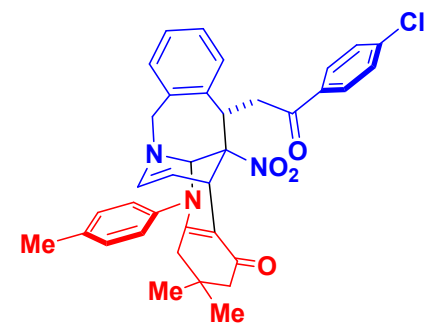

12-(2-(4-chlorophenyl)-2-oxoethyl)-3,3-dimethyl-12a-nitro-5-( $p$-tolyl)-3,4,5,5a,7,12,12a,13-octah ydro-6,13-ethenobenzo[5,6]azepino[2,3-b]quinolin-1(2H)-one (3k)

Yellow solid obtained by column chromatography (petroleum ether/ethyl acetate $=3: 1$ ); $55.6 \mathrm{mg}$, $61 \%$ yield; $\mathrm{dr}>20: 1 ;$ reaction time $=2 \mathrm{~h} ; \mathrm{mp} 238.2-238.8^{\circ} \mathrm{C} ;{ }^{1} \mathrm{H}$ NMR $\left(400 \mathrm{MHz}, \mathrm{CDCl}_{3}\right), \delta$ 
$7.90(\mathrm{~d}, J=8.0 \mathrm{~Hz}, 2 \mathrm{H}), 7.44(\mathrm{~d}, J=12.0 \mathrm{~Hz}, 2 \mathrm{H}), 7.14(\mathrm{q}, J=8.0 \mathrm{~Hz}, 3 \mathrm{H}), 7.07$ (t, $J=8.0 \mathrm{~Hz}$, $1 \mathrm{H}), 6.91\left(\mathrm{dd}, J_{l}=J_{2}=8.0 \mathrm{~Hz}, 4 \mathrm{H}\right), 6.15(\mathrm{~d}, J=4.0 \mathrm{~Hz}, 1 \mathrm{H}), 5.22(\mathrm{t}, J=8.0 \mathrm{~Hz}, 1 \mathrm{H}), 5.04(\mathrm{~s}$, 1H), $4.51\left(\mathrm{dd}, J_{I}=J_{2}=4.0 \mathrm{~Hz}, 1 \mathrm{H}\right), 4.43-4.31(\mathrm{~m}, 3 \mathrm{H}), 3.67-3.55(\mathrm{~m}, 2 \mathrm{H}), 2.36(\mathrm{~s}, 3 \mathrm{H}), 2.14(\mathrm{t}, J$ $=16.0 \mathrm{~Hz}, 2 \mathrm{H}), 1.91(\mathrm{~d}, J=16.0 \mathrm{~Hz}, 1 \mathrm{H}), 1.72(\mathrm{~d}, J=16.0 \mathrm{~Hz}, 1 \mathrm{H}), 0.86(\mathrm{~s}, 3 \mathrm{H}), 0.81(\mathrm{~s}, 3 \mathrm{H})$; ${ }^{13} \mathrm{C}$ NMR $\left(100 \mathrm{MHz}, \mathrm{CDCl}_{3}\right) \delta$ 195.0, 192.3, 156.3, 140.0, 139.2, 138.0, 137.5, 134.9, 134.6, 133.7, 129.8, 129.4, 129.0, 128.1, 127.7, 127.4, 125.6, 108.0, 107.0, 88.8, 73.9, 58.5, 49.3, 40.7, 38.4, 35.3, 32.7, 29.3, 28.5, 27.5, 21.1. IR (KBr) v 3443, 2958, 1686, 1627, 1579, 1392, $741 \mathrm{~cm}^{-1}$. HRMS (ESI) calcd for $\mathrm{C}_{36} \mathrm{H}_{35} \mathrm{ClN}_{3} \mathrm{O}_{4}[\mathrm{M}+\mathrm{H}]^{+}$608.2311, found 608.2290.

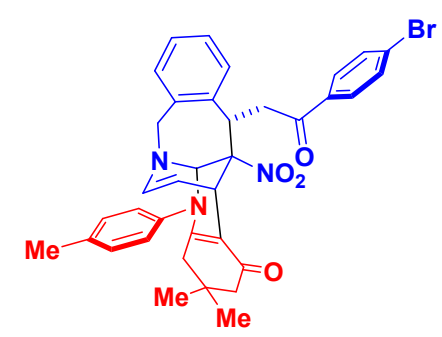

12-(2-(4-bromophenyl)-2-oxoethyl)-3,3-dimethyl-12a-nitro-5-( $p$-tolyl)-3,4,5,5a,7,12,12a, 13-octah ydro-6,13-ethenobenzo[5,6]azepino[2,3-b]quinolin-1(2H)-one (3I)

Yellow solid obtained by column chromatography (petroleum ether/ethyl acetate $=3: 1$ ); $54.3 \mathrm{mg}$, $56 \%$ yield; $\mathrm{dr}>20: 1$; reaction time $=2 \mathrm{~h} ; \mathrm{mp} 236.1-236.7^{\circ} \mathrm{C} ;{ }^{1} \mathrm{H}$ NMR $\left(400 \mathrm{MHz}, \mathrm{CDCl}_{3}\right), \delta$ $7.82(\mathrm{~d}, J=8.0 \mathrm{~Hz}, 2 \mathrm{H}), 7.60(\mathrm{~d}, J=8.0 \mathrm{~Hz}, 2 \mathrm{H}), 7.16-7.11(\mathrm{~m}, 3 \mathrm{H}), 7.07(\mathrm{t}, J=8.0 \mathrm{~Hz}, 1 \mathrm{H}), 6.90$ $\left(\mathrm{dd}, J_{l}=J_{2}=8.0 \mathrm{~Hz}, 4 \mathrm{H}\right), 6.15(\mathrm{~d}, J=4.0 \mathrm{~Hz}, 1 \mathrm{H}), 5.22(\mathrm{t}, J=8.0 \mathrm{~Hz}, 1 \mathrm{H}), 5.04(\mathrm{~s}, 1 \mathrm{H}), 4.51(\mathrm{dd}$, $\left.J_{l}=J_{2}=4.0 \mathrm{~Hz}, 1 \mathrm{H}\right), 4.43-4.31(\mathrm{~m}, 3 \mathrm{H}), 3.66-3.55(\mathrm{~m}, 2 \mathrm{H}), 2.36(\mathrm{~s}, 3 \mathrm{H}), 2.14(\mathrm{t}, J=16.0 \mathrm{~Hz}$, $2 \mathrm{H}), 1.91(\mathrm{~d}, J=16.0 \mathrm{~Hz}, 1 \mathrm{H}), 1.72(\mathrm{~d}, J=16.0 \mathrm{~Hz}, 1 \mathrm{H}), 0.86(\mathrm{~s}, 3 \mathrm{H}), 0.81(\mathrm{~s}, 3 \mathrm{H}) ;{ }^{13} \mathrm{C} \mathrm{NMR}$ $\left(100 \mathrm{MHz}, \mathrm{CDCl}_{3}\right) \delta 195.2,192.3,156.3,139.2,138.0,137.5,135.0,134.9,133.7,132.0,130.3$, $129.5,128.7,128.1,127.7,127.4,125.6,108.0,107.0,88.8,73.9,58.5,49.3,40.7,38.4,35.3$, 32.7, 29.3, 28.5, 27.5, 21.1. IR (KBr) v 3442, 2955, 1628, 1579, 1389, $738 \mathrm{~cm}^{-1}$. HRMS (ESI) calcd for $\mathrm{C}_{36} \mathrm{H}_{35} \mathrm{BrN}_{3} \mathrm{O}_{4}[\mathrm{M}+\mathrm{H}]^{+} 652.1805$, found 652.1820 .

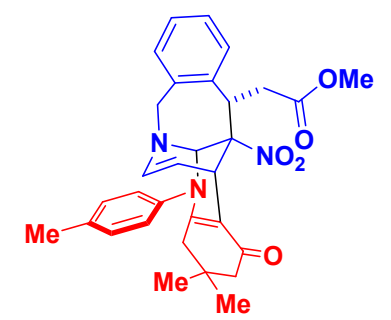

methyl

2-(3,3-dimethyl-12a-nitro-1-oxo-5-( $p$-tolyl)-1,2,3,4,5,5a,7,12,12a,13-decahydro-6,13-ethenobenzo S16 
$[5,6]$ azepino[2,3-b]quinolin-12-yl)acetate(3m)

Yellow solid obtained by column chromatography (petroleum ether/ethyl acetate $=4: 1$ to $3: 1$ ); 22.3mg, 28\% yield; $\mathrm{dr}>20: 1$; reaction time $=2 \mathrm{~h} ; \mathrm{mp} 223.9-224.7^{\circ} \mathrm{C} ;{ }^{1} \mathrm{H}$ NMR $(300 \mathrm{MHz}$, DMSO- $\left.d_{6}\right), \delta 7.21-6.91(\mathrm{~m}, 8 \mathrm{H}), 6.29(\mathrm{~s}, 1 \mathrm{H}), 5.00(\mathrm{~d}, J=24.0 \mathrm{~Hz}, 2 \mathrm{H}), 4.34(\mathrm{~d}, J=21.0 \mathrm{~Hz}$, 3H),3.99 (s, 1H), $3.52(\mathrm{~s}, 3 \mathrm{H}), 3.08(\mathrm{~s}, 1 \mathrm{H}), 2.74(\mathrm{~s}, 1 \mathrm{H}), 2.30(\mathrm{~s}, 3 \mathrm{H}), 2.09-1.94(\mathrm{~m}, 3 \mathrm{H}), 1.63(\mathrm{~d}$, $J=18.0 \mathrm{~Hz}, 1 \mathrm{H}), 0.79$ (s, 3H), 0.69 (s, 3H); ${ }^{13} \mathrm{C} \mathrm{NMR}\left(75 \mathrm{MHz}, \mathrm{DMSO}-d_{6}\right) \delta$ 190.7, 171.1, 154.9, $139.1,137.9,137.6,134.7,134.1,130.6,130.4,128.1,127.9,127.4,126.0,107.2,106.7,88.3$, 79.2, 73.6, 57.9, 51.9, 48.9, 32.6, 31.1, 31.0, 28.6, 26.6, 20.7. IR (KBr) v 3430, 2947, 1747, 1580, 1390, 1169, $758 \mathrm{~cm}^{-1}$. HRMS (ESI) calcd for $\mathrm{C}_{31} \mathrm{H}_{34} \mathrm{~N}_{3} \mathrm{O}_{5}[\mathrm{M}+\mathrm{H}]^{+} 528.2493$, found 528.2492.

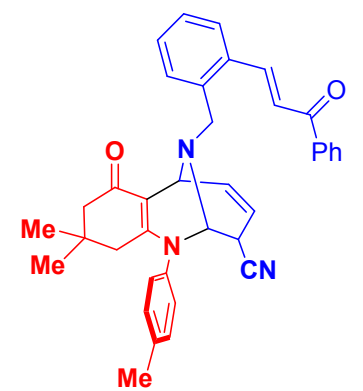

9,9-dimethyl-7-oxo-11-(2-((E)-3-oxo-3-phenylprop-1-en-1-yl)benzyl)-1-( $p$-tolyl)-1,2,3,6,7,8,9,10octahydro-2,6-epiminobenzo[b]azocine-3-carbonitrile (3n)

Yellow solid obtained by column chromatography (petroleum ether/ethyl acetate $=4: 1$ ); $21.1 \mathrm{mg}$,

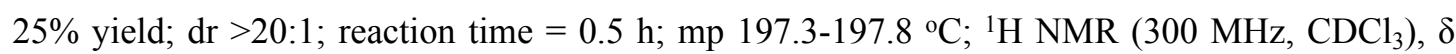
$8.16(\mathrm{~d}, J=15.0 \mathrm{~Hz}, 1 \mathrm{H}), 7.96(\mathrm{t}, J=6.0 \mathrm{~Hz}, 2 \mathrm{H}), 7.70\left(\mathrm{dd}, J_{1}=J_{2}=4.0 \mathrm{~Hz}, 1 \mathrm{H}\right), 7.61-7.58(\mathrm{~m}$, $1 \mathrm{H}), 7.49(\mathrm{t}, J=9.0 \mathrm{~Hz}, 2 \mathrm{H}), 7.36-7.27(\mathrm{~m}, 3 \mathrm{H}), 7.25-7.18(\mathrm{~m}, 4 \mathrm{H}), 6.90\left(\mathrm{dd}, J_{1}=J_{2}=4.0 \mathrm{~Hz}\right.$, 1H), $4.63(\mathrm{~s}, 1 \mathrm{H}), 3.96(\mathrm{~d}, J=3.0 \mathrm{~Hz}, 1 \mathrm{H}), 3.80(\mathrm{q}, J=12.0 \mathrm{~Hz}, 2 \mathrm{H}), 2.59-2.50(\mathrm{~m}, 1 \mathrm{H}), 2.44(\mathrm{dd}$, $\left.J_{l}=J_{2}=4.0 \mathrm{~Hz}, 1 \mathrm{H}\right), 2.38(\mathrm{~s}, 3 \mathrm{H}), 2.29(\mathrm{~d}, J=15.0 \mathrm{~Hz}, 1 \mathrm{H}), 2.22(\mathrm{~m}, 2 \mathrm{H}), 2.07(\mathrm{~d}, J=18.0 \mathrm{~Hz}$, 1H), 1.71 (s, 1H), 1.04 (s, 3H), 0.94 (s, 3H); $\left.{ }^{13} \mathrm{C} \mathrm{NMR} \mathrm{(75} \mathrm{MHz,} \mathrm{CDCl}_{3}\right) \delta$ 194.8, 190.8, 153.6, $147.5,142.5,139.4,138.1,137.7,136.0,135.0,132.8,130.4,130.3,130.0,128.6,128.5,128.3$, 127.2, 124.0, 117.8, 110.8, 107.1, 73.2, 54.2, 50.3, 47.0, 41.1, 34.2, 32.9, 29.7, 28.0, 21.0. IR $(\mathrm{KBr}) v 3429,2927,1621,1568,1401,1257,756 \mathrm{~cm}^{-1}$. HRMS (ESI) calcd for $\mathrm{C}_{37} \mathrm{H}_{36} \mathrm{~N}_{3} \mathrm{O}_{2}$ $[\mathrm{M}+\mathrm{H}]^{+} 554.2802$, found 554.2801. 


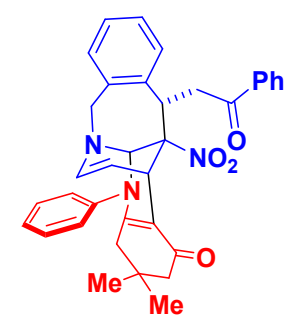

3,3-dimethyl-12a-nitro-12-(2-oxo-2-phenylethyl)-5-phenyl-3,4,5,5a,7,12,12a,13-octahydro-6,13-et henobenzo[5,6]azepino[2,3-b]quinolin-1(2H)-one (3o)

Yellow solid obtained by column chromatography (petroleum ether/ethyl acetate $=3: 1$ ); $67.0 \mathrm{mg}$, $80 \%$ yield; $\mathrm{dr}>20: 1 ;$ reaction time $=2 \mathrm{~h} ; \mathrm{mp} 153.1-153.9^{\circ} \mathrm{C} ;{ }^{1} \mathrm{H}$ NMR $\left(400 \mathrm{MHz}, \mathrm{CDCl}_{3}\right), \delta 7.97$ $(\mathrm{d}, J=8.0 \mathrm{~Hz}, 2 \mathrm{H}), 7.60(\mathrm{t}, J=8.0 \mathrm{~Hz}, 1 \mathrm{H}), 7.48(\mathrm{t}, J=8.0 \mathrm{~Hz}, 2 \mathrm{H}), 7.37(\mathrm{t}, J=8.0 \mathrm{~Hz}, 3 \mathrm{H}), 7.11$ (tt, $\left.J_{I}=J_{2}=8.0 \mathrm{~Hz}, 4 \mathrm{H}\right), 6.97(\mathrm{~d}, J=8.0 \mathrm{~Hz}, 1 \mathrm{H}), 6.90(\mathrm{~d}, J=8.0 \mathrm{~Hz}, 1 \mathrm{H}), 6.17(\mathrm{~d}, J=8.0 \mathrm{~Hz}, 1 \mathrm{H})$, $5.25(\mathrm{t}, J=8.0 \mathrm{~Hz}, 1 \mathrm{H}), 5.10(\mathrm{~s}, 1 \mathrm{H}), 4.54(\mathrm{~d}, J=4.0 \mathrm{~Hz}, 1 \mathrm{H}), 4.45-4.31(\mathrm{~m}, 3 \mathrm{H}), 3.73-3.59(\mathrm{~m}$, 2H), 2.16 (t, $J=16.0 \mathrm{~Hz}, 2 \mathrm{H}), 1.92(\mathrm{~d}, J=16.0 \mathrm{~Hz}, 1 \mathrm{H}), 1.72(\mathrm{~d}, J=16.0 \mathrm{~Hz}, 1 \mathrm{H}), 0.88(\mathrm{~s}, 3 \mathrm{H})$, $0.82(\mathrm{~s}, 3 \mathrm{H}) ;{ }^{13} \mathrm{C}$ NMR $\left(100 \mathrm{MHz}, \mathrm{CDCl}_{3}\right) \delta 196.2,192.4,156.1,141.9,137.5,136.4,135.2$, $133.8,133.5,128.7,128.1,128.1,127.7,127.5,125.8,108.1,107.4,88.9,74.1,58.6,49.4,40.8$, $38.5,35.4,32.8,29.3,28.6,27.6$, two carbons missing in the aromatic region. IR (KBr) $v$ 3441, 2955, 1628, 1576, 1390, $738 \mathrm{~cm}^{-1}$. HRMS (ESI) calcd for $\mathrm{C}_{35} \mathrm{H}_{34} \mathrm{~N}_{3} \mathrm{O}_{4}[\mathrm{M}+\mathrm{H}]^{+} 560.2544$, found 560.2550.

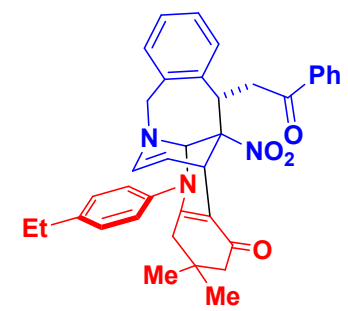

5-(4-ethylphenyl)-3,3-dimethyl-12a-nitro-12-(2-oxo-2-phenylethyl)-3,4,5,5a,7,12,12a,13-octahydr o-6,13-ethenobenzo[5,6]azepino[2,3-b]quinolin-1(2H)-one (3p)

Yellow solid obtained by column chromatography (petroleum ether/ethyl acetate $=3: 1$ ); $38.3 \mathrm{mg}$, $43 \%$ yield; $\mathrm{dr}>20: 1 ;$ reaction time $=2 \mathrm{~h} ; \mathrm{mp} 158.1-158.9^{\circ} \mathrm{C} ;{ }^{1} \mathrm{H}$ NMR $\left(400 \mathrm{MHz}, \mathrm{CDCl}_{3}\right), \delta 7.95$ (d, $J=4.0 \mathrm{~Hz}, 2 \mathrm{H}), 7.57$ (t, $J=4.0 \mathrm{~Hz}, 1 \mathrm{H}), 7.46(\mathrm{~d}, J=4.0 \mathrm{~Hz}, 2 \mathrm{H}), 7.17-6.89(\mathrm{~m}, 8 \mathrm{H}), 6.15(\mathrm{~s}$, 1H), $5.23(\mathrm{~s}, 1 \mathrm{H}), 5.07(\mathrm{~s}, 1 \mathrm{H}), 4.54(\mathrm{~s}, 1 \mathrm{H}), 4.37(\mathrm{q}, J=16.0 \mathrm{~Hz}, 3 \mathrm{H}), 3.72-3.58(\mathrm{~m}, 2 \mathrm{H}), 2.66(\mathrm{~d}$, $J=8.0 \mathrm{~Hz}, 2 \mathrm{H}), 2.14(\mathrm{~s}, 2 \mathrm{H}), 1.92(\mathrm{~d}, J=16.0 \mathrm{~Hz}, 1 \mathrm{H}), 1.73(\mathrm{~d}, J=16.0 \mathrm{~Hz}, 1 \mathrm{H}), 1.25(\mathrm{t}, J=8.0$ $\mathrm{Hz}, 3 \mathrm{H}), 0.87$ (s, 3H), 0.82 (s, 3H); ${ }^{13} \mathrm{C}$ NMR (100 MHz, $\left.\mathrm{CDCl}_{3}\right) \delta 196.1,192.2,156.3,144.1$, $139.4,137.5,136.3,135.1,133.7,133.5,128.9,128.6,128.0,128.0,127.6,127.3,125.7,108.0$, 
107.1, 88.9, 74.0, 58.5, 49.4, 40.7, 38.5, 35.3, 32.7, 29.3, 28.5, 28.3, 27.5, 15.1. IR (KBr) v 3437, $2958,1626,1578,1392,1237,738 \mathrm{~cm}^{-1}$. HRMS (ESI) calcd for $\mathrm{C}_{37} \mathrm{H}_{38} \mathrm{~N}_{3} \mathrm{O}_{4}[\mathrm{M}+\mathrm{H}]^{+}$588.2857, found 588.2865

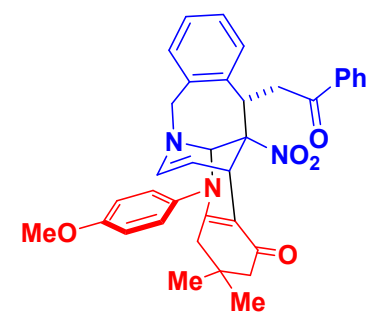

5-(4-methoxyphenyl)-3,3-dimethyl-12a-nitro-12-(2-oxo-2-phenylethyl)-3,4,5,5a,7,12,12a,13-octah ydro-6,13-ethenobenzo[5,6]azepino[2,3-b]quinolin-1(2H)-one (3q)

Yellow solid obtained by column chromatography (petroleum ether/ethyl acetate $=3: 1$ ); $60.1 \mathrm{mg}$, $68 \%$ yield; $\mathrm{dr}>20: 1 ;$ reaction time $=2 \mathrm{~h} ; \mathrm{mp} 214.2-214.9^{\circ} \mathrm{C} ;{ }^{1} \mathrm{H}$ NMR $\left(400 \mathrm{MHz}, \mathrm{CDCl}_{3}\right), \delta 7.96$ (d, $J=8.0 \mathrm{~Hz}, 2 \mathrm{H}), 7.59(\mathrm{t}, J=8.0 \mathrm{~Hz}, 1 \mathrm{H}), 7.47(\mathrm{t}, J=8.0 \mathrm{~Hz}, 2 \mathrm{H}), 7.14(\mathrm{t}, J=8.0 \mathrm{~Hz}, 1 \mathrm{H}), 7.07$ $(\mathrm{t}, J=8.0 \mathrm{~Hz}, 2 \mathrm{H}), 6.97(\mathrm{~d}, J=8.0 \mathrm{~Hz}, 1 \mathrm{H}), 6.89(\mathrm{~d}, J=8.0 \mathrm{~Hz}, 4 \mathrm{H}), 6.16(\mathrm{~d}, J=4.0 \mathrm{~Hz}, 1 \mathrm{H})$, $5.23(\mathrm{t}, J=8.0 \mathrm{~Hz}, 1 \mathrm{H}), 5.01(\mathrm{~s}, 1 \mathrm{H}), 4.53(\mathrm{~d}, J=8.0 \mathrm{~Hz}, 1 \mathrm{H}), 4.45-4.33(\mathrm{~m}, 3 \mathrm{H}), 3.82(\mathrm{~s}, 3 \mathrm{H})$, 3.72-3.58 (m, 2H), 2.14 (t, $J=16.0 \mathrm{~Hz}, 2 \mathrm{H}), 1.89(\mathrm{~d}, J=16.0 \mathrm{~Hz}, 1 \mathrm{H}), 1.74(\mathrm{~d}, J=16.0 \mathrm{~Hz}, 1 \mathrm{H})$, $0.87(\mathrm{~s}, 3 \mathrm{H}), 0.83(\mathrm{~s}, 3 \mathrm{H}) ;{ }^{13} \mathrm{C} \mathrm{NMR}\left(100 \mathrm{MHz}, \mathrm{CDCl}_{3}\right) \delta$ 196.2, 192.3, 159.0, 156.6, 137.5, 136.3, 135.1, 134.6, 133.7, 133.5, 128.7, 128.1, 128.0, 127.7, 127.4, 125.8, 125.8, 108.1, 107.0, 88.9, 74.2, 58.6, 55.4, 49.4, 40.7, 38.5, 35.3, 32.6, 29.3, 28.5, 27.7. IR (KBr) v 3441, 2951, 1623 , 1577, 1392, 1240, $734 \mathrm{~cm}^{-1}$. HRMS (ESI) calcd for $\mathrm{C}_{36} \mathrm{H}_{36} \mathrm{~N}_{3} \mathrm{O}_{5}[\mathrm{M}+\mathrm{H}]^{+}$590.2649, found 590.2651.

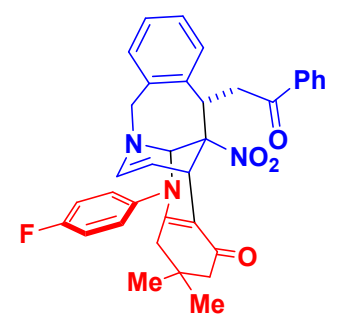

5-(4-fluorophenyl)-3,3-dimethyl-12a-nitro-12-(2-oxo-2-phenylethyl)-3,4,5,5a,7,12,12a,13-octahyd ro-6,13-ethenobenzo[5,6]azepino[2,3-b]quinolin-1(2H)-one (3r)

Yellow solid obtained by column chromatography (petroleum ether/ethyl acetate $=3: 1$ ); $63.5 \mathrm{mg}$, $73 \%$ yield; $\mathrm{dr}>20: 1 ;$ reaction time $=2 \mathrm{~h} ; \mathrm{mp} 223.1-223.6^{\circ} \mathrm{C} ;{ }^{1} \mathrm{H}$ NMR $\left(400 \mathrm{MHz}, \mathrm{CDCl}_{3}\right), \delta 7.95$ $(\mathrm{d}, J=4.0 \mathrm{~Hz}, 2 \mathrm{H}), 7.58(\mathrm{t}, J=8.0 \mathrm{~Hz}, 1 \mathrm{H}), 7.46(\mathrm{t}, J=8.0 \mathrm{~Hz}, 2 \mathrm{H}), 7.16-6.97(\mathrm{~m}, 7 \mathrm{H}), 6.89(\mathrm{~d}, J$ $=8.0 \mathrm{~Hz}, 1 \mathrm{H}), 6.16(\mathrm{~d}, J=4.0 \mathrm{~Hz}, 1 \mathrm{H}), 5.24(\mathrm{t}, J=8.0 \mathrm{~Hz}, 1 \mathrm{H}), 5.01(\mathrm{~s}, 1 \mathrm{H}), 4.53(\mathrm{~d}, J=4.0 \mathrm{~Hz}$, 
1H), 4.45-4.31 (m, 3H), 3.72-3.57 (m, 2H), 2.14 (t, $J=16.0 \mathrm{~Hz}, 2 \mathrm{H}), 1.87(\mathrm{~d}, J=16.0 \mathrm{~Hz}, 1 \mathrm{H})$, $1.71(\mathrm{~d}, J=16.0 \mathrm{~Hz}, 1 \mathrm{H}), 0.87(\mathrm{~s}, 3 \mathrm{H}), 0.82(\mathrm{~s}, 3 \mathrm{H}) ;{ }^{13} \mathrm{C} \mathrm{NMR}\left(100 \mathrm{MHz}, \mathrm{CDCl}_{3}\right) \delta 196.1,192.4$, $161.9(\mathrm{~d}, J=247.0 \mathrm{~Hz}, 1 \mathrm{C}), 155.8,133.8,133.8,137.3,136.3,135.0,133.7,133.5,128.7,128.1$, $128.0,127.7,127.5,125.7,108.2,107.5,88.8,74.1,58.5,49.3,40.7,38.4,35.2,32.6,29.2,28.5$, 27.6. IR $(\mathrm{KBr}) v 3445,2954,1626,1581,1391,1222,732 \mathrm{~cm}^{-1}$. HRMS (ESI) calcd for $\mathrm{C}_{35} \mathrm{H}_{33} \mathrm{FN}_{3} \mathrm{O}_{4}[\mathrm{M}+\mathrm{H}]^{+}$578.2450, found 578.2447.

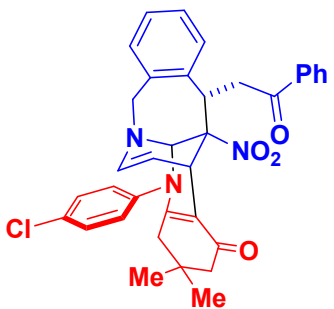

5-(4-chlorophenyl)-3,3-dimethyl-12a-nitro-12-(2-oxo-2-phenylethyl)-3,4,5,5a,7,12,12a,13-octahy dro-6,13-ethenobenzo[5,6]azepino[2,3-b]quinolin-1(2H)-one (3s)

Yellow solid obtained by column chromatography (petroleum ether/ethyl acetate $=3: 1$ ); $66.9 \mathrm{mg}$, $75 \%$ yield; $\mathrm{dr}>20: 1 ;$ reaction time $=2 \mathrm{~h} ; \mathrm{mp} 215.6-216.1^{\circ} \mathrm{C} ;{ }^{1} \mathrm{H}$ NMR $\left(400 \mathrm{MHz}, \mathrm{CDCl}_{3}\right), \delta 7.96$ $(\mathrm{d}, J=4.0 \mathrm{~Hz}, 2 \mathrm{H}), 7.58(\mathrm{t}, J=8.0 \mathrm{~Hz}, 1 \mathrm{H}), 7.47(\mathrm{t}, J=8.0 \mathrm{~Hz}, 2 \mathrm{H}), 7.34(\mathrm{~d}, J=4.0 \mathrm{~Hz}, 2 \mathrm{H})$, 7.16-6.88 (m, 6H), $6.15(\mathrm{~d}, J=4.0 \mathrm{~Hz}, 1 \mathrm{H}), 5.23(\mathrm{t}, J=8.0 \mathrm{~Hz}, 1 \mathrm{H}), 5.03(\mathrm{~s}, 1 \mathrm{H}), 4.54-4.32(\mathrm{~m}$, 4H), 3.72-3.46 (m, 2H), $2.15(\mathrm{t}, J=16.0 \mathrm{~Hz}, 2 \mathrm{H}), 1.90(\mathrm{~d}, J=16.0 \mathrm{~Hz}, 1 \mathrm{H}), 1.70(\mathrm{~d}, J=16.0 \mathrm{~Hz}$, 1H), 0.88 (s, 3H), 0.82 (s, 3H); ${ }^{13} \mathrm{C}$ NMR (100 MHz, $\left.\mathrm{CDCl}_{3}\right) \delta$ 196.0, 192.4, 155.5, 140.3, 137.3, $136.2,135.0,133.9,133.7,133.5,129.6,128.7,128.1,128.0,127.7,127.5,125.7,108.2,107.8$, $88.8,74.0,58.5,49.3,40.7,38.4,35.2,32.7,29.2,28.5,27.5$. IR (KBr) $v 3437,2926,1631,1585$, 1390, $758 \mathrm{~cm}^{-1}$. HRMS (ESI) calcd for $\mathrm{C}_{35} \mathrm{H}_{33} \mathrm{ClN}_{3} \mathrm{O}_{4}[\mathrm{M}+\mathrm{H}]^{+}$594.2154, found 594.2149.

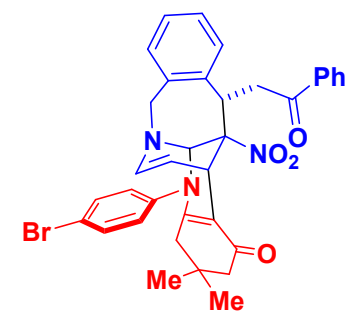

5-(4-bromophenyl)-3,3-dimethyl-12a-nitro-12-(2-oxo-2-phenylethyl)-3,4,5,5a,7,12,12a,13-octahy dro-6,13-ethenobenzo[5,6]azepino[2,3-b]quinolin-1(2H)-one (3t)

Yellow solid obtained by column chromatography (petroleum ether/ethyl acetate $=3: 1$ ); $62.8 \mathrm{mg}$, $66 \%$ yield; $\mathrm{dr}>20: 1 ;$ reaction time $=2 \mathrm{~h} ; \mathrm{mp} 150.4-151.2^{\circ} \mathrm{C} ;{ }^{1} \mathrm{H}$ NMR $\left(400 \mathrm{MHz}, \mathrm{CDCl}_{3}\right), \delta 7.96$ $(\mathrm{d}, J=8.0 \mathrm{~Hz}, 2 \mathrm{H}), 7.60(\mathrm{t}, J=8.0 \mathrm{~Hz}, 1 \mathrm{H}), 7.48\left(\mathrm{dd}, J_{l}=J_{2}=8.0 \mathrm{~Hz}, 4 \mathrm{H}\right), 7.15(\mathrm{t}, J=8.0 \mathrm{~Hz}$, S20 
1H), $7.08(\mathrm{t}, J=8.0 \mathrm{~Hz}, 1 \mathrm{H}), 6.99(\mathrm{~d}, J=4.0 \mathrm{~Hz}, 2 \mathrm{H}), 6.89(\mathrm{~d}, J=8.0 \mathrm{~Hz}, 2 \mathrm{H}), 6.15(\mathrm{~d}, J=4.0$ $\mathrm{Hz}, 1 \mathrm{H}), 5.23(\mathrm{t}, J=8.0 \mathrm{~Hz}, 1 \mathrm{H}), 5.04(\mathrm{~s}, 1 \mathrm{H}), 4.53(\mathrm{~d}, J=8.0 \mathrm{~Hz}, 1 \mathrm{H}), 3.38\left(\mathrm{dd}, J_{I}=J_{2}=16.0\right.$ $\mathrm{Hz}, 3 \mathrm{H}), 3.72-3.57(\mathrm{~m}, 2 \mathrm{H}), 2.15(\mathrm{t}, J=16.0 \mathrm{~Hz}, 2 \mathrm{H}), 1.90(\mathrm{~d}, J=16.0 \mathrm{~Hz}, 1 \mathrm{H}), 1.70(\mathrm{~d}, J=16.0$ $\mathrm{Hz}, 1 \mathrm{H}), 0.88$ (s, 3H), 0.82 (s, 3H); ${ }^{13} \mathrm{C}$ NMR (100 MHz, $\left.\mathrm{CDCl}_{3}\right) \delta$ 196.0, 192.5, 155.4, 140.9, $137.3,136.3,135.0,133.7,133.6,133.1,128.7,128.1,128.0,127.8,127.5,125.7,122.0,108.2$, $107.8,88.8,74.0,58.5,49.3,40.8,38.5,35.2,32.8,29.2,28.6,27.5$. IR (KBr) v 3441, 2954, 1629 , 1582, 1389, $737 \mathrm{~cm}^{-1}$. HRMS (ESI) calcd for $\mathrm{C}_{35} \mathrm{H}_{33} \mathrm{BrN}_{3} \mathrm{O}_{4}[\mathrm{M}+\mathrm{H}]^{+} 638.1649$, found 638.1646 .

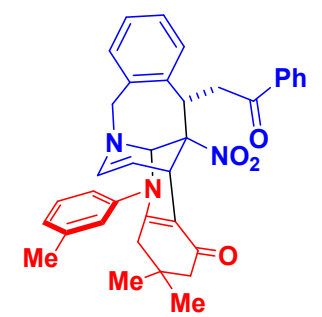

3,3-dimethyl-12a-nitro-12-(2-oxo-2-phenylethyl)-5-( $m$-tolyl)-3,4,5,5a,7,12,12a,13-octahydro-6,13 -ethenobenzo[5,6]azepino[2,3-b]quinolin-1(2H)-one (3u)

Yellow solid obtained by column chromatography (petroleum ether/ethyl acetate $=3: 1$ ); $60.1 \mathrm{mg}$, $70 \%$ yield; $\mathrm{dr}>20: 1 ;$ reaction time $=2 \mathrm{~h} ; \mathrm{mp} 237.3-237.8^{\circ} \mathrm{C} ;{ }^{1} \mathrm{H}$ NMR $\left(400 \mathrm{MHz}, \mathrm{CDCl}_{3}\right), \delta 7.95$ $(\mathrm{d}, J=8.0 \mathrm{~Hz}, 2 \mathrm{H}), 7.57(\mathrm{t}, J=8.0 \mathrm{~Hz}, 1 \mathrm{H}), 7.45(\mathrm{t}, J=8.0 \mathrm{~Hz}, 2 \mathrm{H}), 7.24(\mathrm{~s}, 1 \mathrm{H}), 7.12\left(\mathrm{dd}, J_{l}\right.$ $\left.=8.0 \mathrm{~Hz}, J_{2}=4.0 \mathrm{~Hz}, 2 \mathrm{H}\right), 7.05(\mathrm{t}, J=8.0 \mathrm{~Hz}, 1 \mathrm{H}), 6.92\left(\mathrm{dd}, J_{l}=J_{2}=8.0 \mathrm{~Hz}, 4 \mathrm{H}\right), 6.16(\mathrm{~d}, J=4.0$ $\mathrm{Hz}, 1 \mathrm{H}), 5.23(\mathrm{t}, J=8.0 \mathrm{~Hz}, 1 \mathrm{H}), 5.08(\mathrm{~s}, 1 \mathrm{H}), 4.54(\mathrm{~d}, J=4.0 \mathrm{~Hz}, 1 \mathrm{H}), 4.43-4.31(\mathrm{~m}, 3 \mathrm{H})$, 3.73-3.58 (m, 2H), $2.34(\mathrm{~d}, J=8.0 \mathrm{~Hz}, 3 \mathrm{H}), 2.14(\mathrm{t}, J=16.0 \mathrm{~Hz}, 2 \mathrm{H}), 1.92(\mathrm{~d}, J=16.0 \mathrm{~Hz}, 1 \mathrm{H})$, $1.72(\mathrm{~d}, J=16.0 \mathrm{~Hz}, 1 \mathrm{H}), 0.87$ (s, 3H), $0.81(\mathrm{~s}, 3 \mathrm{H}) ;{ }^{13} \mathrm{C} \mathrm{NMR}\left(100 \mathrm{MHz}, \mathrm{CDCl}_{3}\right) \delta 196.1,192.2$, $156.1,141.7,137.5,136.3,135.0,133.7,133.4,129.6,129.3,128.9,128.6,128.0,127.9,127.6$, $127.4,127.3,125.7,107.9,107.1,88.8,73.9,58.5,49.3,40.7,38.4,35.3,32.6,29.2,28.5,27.4$, 21.2. IR (KBr) v 3444, 2954, 1627, 1578, 1390, $732 \mathrm{~cm}^{-1}$. HRMS (ESI) calcd for $\mathrm{C}_{36} \mathrm{H}_{36} \mathrm{~N}_{3} \mathrm{O}_{4}$ $[\mathrm{M}+\mathrm{H}]^{+}$574.2700, found 574.2707.

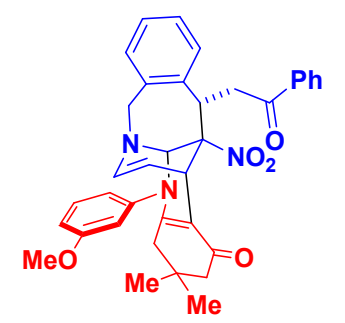

5-(3-methoxyphenyl)-3,3-dimethyl-12a-nitro-12-(2-oxo-2-phenylethyl)-3,4,5,5a,7,12,12a,13-octah ydro-6,13-ethenobenzo[5,6]azepino[2,3-b]quinolin-1(2H)-one (3v) 
Yellow solid obtained by column chromatography (petroleum ether/ethyl acetate $=3: 1$ ); $60.2 \mathrm{mg}$, $68 \%$ yield; $\mathrm{dr}>20: 1 ;$ reaction time $=2 \mathrm{~h} ; \mathrm{mp} 260.2-261.1^{\circ} \mathrm{C} ;{ }^{1} \mathrm{H}$ NMR $\left(400 \mathrm{MHz}, \mathrm{CDCl}_{3}\right), \delta 7.77$ $(\mathrm{d}, J=4.0 \mathrm{~Hz}, 2 \mathrm{H}), 7.38(\mathrm{~d}, J=8.0 \mathrm{~Hz}, 1 \mathrm{H}), 7.28(\mathrm{t}, J=8.0 \mathrm{~Hz}, 2 \mathrm{H}), 7.10(\mathrm{~s}, 1 \mathrm{H}), 6.94-6.86(\mathrm{~m}$, 2H), $6.78(\mathrm{~d}, J=8.0 \mathrm{~Hz}, 1 \mathrm{H}), 6.71(\mathrm{t}, J=8.0 \mathrm{~Hz}, 2 \mathrm{H}), 6.46(\mathrm{br}, 2 \mathrm{H}), 5.98(\mathrm{~d}, J=4.0 \mathrm{~Hz}, 1 \mathrm{H}), 5.06$ (t, $J=4.0 \mathrm{~Hz}, 1 \mathrm{H}), 4.93(\mathrm{~s}, 1 \mathrm{H}), 4.36(\mathrm{~d}, J=4.0 \mathrm{~Hz}, 1 \mathrm{H}), 4.25-4.20(\mathrm{~m}, 3 \mathrm{H}), 3.59-3.40(\mathrm{~m}, 5 \mathrm{H})$, $1.97(\mathrm{t}, J=16.0 \mathrm{~Hz}, 2 \mathrm{H}), 1.79(\mathrm{~d}, J=16.0 \mathrm{~Hz}, 1 \mathrm{H}), 1.59(\mathrm{~d}, J=16.0 \mathrm{~Hz}, 1 \mathrm{H}), 0.70(\mathrm{~s}, 3 \mathrm{H}), 0.64$ (s, 3H); ${ }^{13} \mathrm{C}$ NMR $\left(100 \mathrm{MHz}, \mathrm{CDCl}_{3}\right) \delta 196.0,192.2,160.0,155.8,142.9,137.4,136.2,135.0$, 133.6, 133.4, 130.2, 128.6, 128.0, 127.9, 127.6, 127.3, 125.7, 119.0, 113.2, 107.9, 107.3, 88.7, 73.8, 58.4, 55.3, 49.3, 40.5, 38.4, 35.2, 32.6, 29.2, 28.4, 27.4. IR (KBr) v 3440, 2952, 1625, 1575, 1391, $731 \mathrm{~cm}^{-1}$. HRMS (ESI) calcd for $\mathrm{C}_{36} \mathrm{H}_{36} \mathrm{~N}_{3} \mathrm{O}_{5}[\mathrm{M}+\mathrm{H}]^{+}$590.2649, found 590.2664.

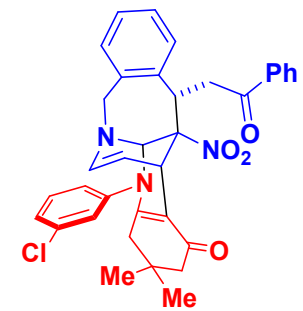

5-(3-chlorophenyl)-3,3-dimethyl-12a-nitro-12-(2-oxo-2-phenylethyl)-3,4,5,5a,7,12,12a,13-octahy dro-6,13-ethenobenzo[5,6]azepino[2,3-b]quinolin-1(2H)-one (3w)

Yellow solid obtained by column chromatography (petroleum ether/ethyl acetate $=3: 1$ ); $67.3 \mathrm{mg}$, $76 \%$ yield; $\mathrm{dr}>20: 1 ;$ reaction time $=2 \mathrm{~h} ; \mathrm{mp} 229.1-229.8^{\circ} \mathrm{C} ;{ }^{1} \mathrm{H}$ NMR $\left(400 \mathrm{MHz}, \mathrm{CDCl}_{3}\right), \delta 7.94$ $(\mathrm{d}, J=8.0 \mathrm{~Hz}, 2 \mathrm{H}), 7.55(\mathrm{~d}, J=8.0 \mathrm{~Hz}, 1 \mathrm{H}), 7.45(\mathrm{~d}, J=8.0 \mathrm{~Hz}, 2 \mathrm{H}), 7.31(\mathrm{~s}, 2 \mathrm{H}), 7.12-6.88(\mathrm{~m}$, $6 \mathrm{H}), 6.15(\mathrm{~d}, J=4.0 \mathrm{~Hz}, 1 \mathrm{H}), 5.23(\mathrm{t}, J=4.0 \mathrm{~Hz}, 1 \mathrm{H}), 5.05(\mathrm{~s}, 1 \mathrm{H}), 4.53(\mathrm{~d}, J=8.0 \mathrm{~Hz}, 1 \mathrm{H})$, 4.43-4.31 (m, 3H), 3.72-3.56 (m, 2H), $2.14(\mathrm{t}, J=16.0 \mathrm{~Hz}, 2 \mathrm{H}), 1.90(\mathrm{~d}, J=16.0 \mathrm{~Hz}, 1 \mathrm{H}), 1.70(\mathrm{~d}$, $J=16.0 \mathrm{~Hz}, 1 \mathrm{H}), 0.87(\mathrm{~s}, 3 \mathrm{H}), 0.81(\mathrm{~s}, 3 \mathrm{H}) ;{ }^{13} \mathrm{C} \mathrm{NMR}\left(100 \mathrm{MHz}, \mathrm{CDCl}_{3}\right) \delta$ 195.9, 192.3, 155.1, $142.9,137.2,136.2,134.9,134.8,133.6,133.4,130.7,130.5,128.6,128.4,128.0,127.9,127.7$, 127.4, 125.7, 108.0, 107.9, 88.7, 73.9, 58.4, 49.2, 40.6, 38.4, 35.1, 32.7, 29.1, 28.5, 27.4. IR (KBr) $v$ 3455, 2954, 1630, 1577, 1387, $759 \mathrm{~cm}^{-1}$. HRMS (ESI) calcd for $\mathrm{C}_{35} \mathrm{H}_{33} \mathrm{ClN}_{3} \mathrm{O}_{4}[\mathrm{M}+\mathrm{H}]^{+}$ 594.2154 , found 594.2158. 


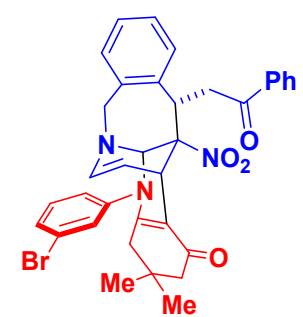

5-(3-bromophenyl)-3,3-dimethyl-12a-nitro-12-(2-oxo-2-phenylethyl)-3,4,5,5a,7,12,12a,13-octahy dro-6,13-ethenobenzo[5,6]azepino[2,3-b]quinolin-1(2H)-one (3x)

Yellow solid obtained by column chromatography (petroleum ether/ethyl acetate $=3: 1$ ); $71.3 \mathrm{mg}$, $74 \%$ yield; $\mathrm{dr}>20: 1 ;$ reaction time $=2 \mathrm{~h} ; \mathrm{mp} 251.3-252.1^{\circ} \mathrm{C} ;{ }^{1} \mathrm{H}$ NMR $\left(400 \mathrm{MHz}, \mathrm{CDCl}_{3}\right), \delta$ $7.89(\mathrm{~d}, J=4.0 \mathrm{~Hz}, 2 \mathrm{H}), 7.53$ (t, $J=8.0 \mathrm{~Hz}, 1 \mathrm{H}), 7.42(\mathrm{q}, J=8.0 \mathrm{~Hz}, 3 \mathrm{H}), 7.21-7.92(\mathrm{~m}, 6 \mathrm{H}), 6.83$ $(\mathrm{d}, J=8.0 \mathrm{~Hz}, 1 \mathrm{H}), 6.10(\mathrm{~d}, J=4.0 \mathrm{~Hz}, 1 \mathrm{H}), 6.17(\mathrm{t}, J=8.0 \mathrm{~Hz}, 1 \mathrm{H}), 4.99(\mathrm{t}, J=8.0 \mathrm{~Hz}, 1 \mathrm{H})$, $4.45(\mathrm{~d}, J=8.0 \mathrm{~Hz}, 1 \mathrm{H}), 4.33\left(\mathrm{dd}, J_{I}=J_{2}=16.0 \mathrm{~Hz}, 3 \mathrm{H}\right), 3.66-3.50(\mathrm{~m}, 2 \mathrm{H}), 2.09(\mathrm{t}, J=16.0 \mathrm{~Hz}$, 2H), $1.84(\mathrm{~d}, J=16.0 \mathrm{~Hz}, 1 \mathrm{H}), 1.64(\mathrm{~d}, J=16.0 \mathrm{~Hz}, 1 \mathrm{H}), 0.82(\mathrm{~s}, 3 \mathrm{H}), 0.77(\mathrm{~s}, 3 \mathrm{H}) ;{ }^{13} \mathrm{C} \mathrm{NMR}$ $\left(100 \mathrm{MHz}, \mathrm{CDCl}_{3}\right) \delta 196.1,192.5,155.3,143.2,137.3,136.3,135.0,133.7,133.6,131.5,131.0$, $130.9,128.7,128.2,128.0,127.8,127.6,125.8,122.8,108.2,108.1,88.8,74.0,58.6,49.4,40.8$, 38.5, 35.3, 32.8, 29.3, 28.6, 27.5. IR (KBr) v 3443, 2953, 1629, 1572, 1388, $692 \mathrm{~cm}^{-1}$. HRMS (ESI) calcd for $\mathrm{C}_{35} \mathrm{H}_{33} \mathrm{BrN}_{3} \mathrm{O}_{4}[\mathrm{M}+\mathrm{H}]^{+}$638.1649, found 638.1663.

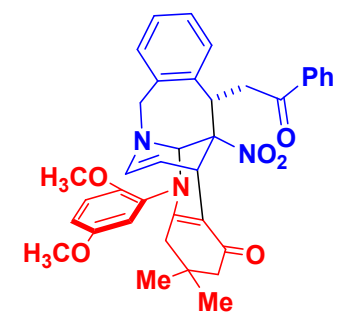

5-(2,5-dimethoxyphenyl)-3,3-dimethyl-12a-nitro-12-(2-oxo-2-phenylethyl)-3,4,5,5a,7,12,12a,13-o ctahydro-6,13-ethenobenzo[5,6]azepino[2,3-b]quinolin-1(2H)-one (3y)

Yellow solid obtained by column chromatography (petroleum ether/ethyl acetate $=3: 1$ ); $49.0 \mathrm{mg}$, $53 \%$ yield; $\mathrm{dr}=3: 1$ (inseperable isomers); reaction time $=2 \mathrm{~h} ; \mathrm{mp} 223.8-224.8^{\circ} \mathrm{C} ;{ }^{1} \mathrm{H}$ NMR $(400$ $\left.\mathrm{MHz}, \mathrm{CDCl}_{3}\right), \delta 7.93(\mathrm{~d}, J=8.0 \mathrm{~Hz}, 2 \mathrm{H}), 7.56(\mathrm{t}, J=8.0 \mathrm{~Hz}, 1 \mathrm{H}), 7.44(\mathrm{t}, J=8.0 \mathrm{~Hz}, 2 \mathrm{H})$, 7.13-7.00 (m, 2H), 6.94-6.81 (m, 3H), $6.78(\mathrm{~d}, J=12.0 \mathrm{~Hz}, 1 \mathrm{H}), 6.73(\mathrm{~d}, J=4.0 \mathrm{~Hz}, 1 \mathrm{H}), 6.12$ $\left(\mathrm{dd}, J_{1}=4.0 \mathrm{~Hz}, J_{2}=8.0 \mathrm{~Hz}, 1 \mathrm{H}\right), 5.21(\mathrm{t}, J=8.0 \mathrm{~Hz}, 1 \mathrm{H}), 4.89(\mathrm{~s}, 1 \mathrm{H}), 4.52-4.46(\mathrm{~m}, 1 \mathrm{H})$, 4.39-4.25 (m, 3H), $3.77(\mathrm{~s}, 3 \mathrm{H}), 3.65-3.58(\mathrm{~m}, 2 \mathrm{H}), 3.51(\mathrm{~s}, 3 \mathrm{H}), 2.20-2.07(\mathrm{~m}, 2 \mathrm{H}), 1.91(\mathrm{~d}, J=$ $16.0 \mathrm{~Hz}, 1 \mathrm{H}), 1.75(\mathrm{~d}, J=16.0 \mathrm{~Hz}, 1 \mathrm{H}), 0.86(\mathrm{~s}, 3 \mathrm{H}), 0.84(\mathrm{~s}, 3 \mathrm{H}) ;{ }^{13} \mathrm{C} \mathrm{NMR}\left(100 \mathrm{MHz}, \mathrm{CDCl}_{3}\right) \delta$ $196.1,192.4,156.6,153.2,149.5,137.4,136.3,135.5,135.2,130.0,128.6,127.9,127.8,127.2$, 
127.1, 126.1, 117.1, 113.9, 112.7, 108.2, 107.4, 88.4, 72.6, 58.4, 55.7, 55.7, 49.5, 39.7, 38.7, 35.2, 32.6, 28.9, 28.6, 27.5. IR (KBr) v 3422, 2952, 1688, 1626, 1582, 1388, 1037, $732 \mathrm{~cm}^{-1}$. HRMS (ESI) calcd for $\mathrm{C}_{37} \mathrm{H}_{38} \mathrm{~N}_{3} \mathrm{O}_{6}[\mathrm{M}+\mathrm{H}]^{+} 620.2755$, found 620.2749 .

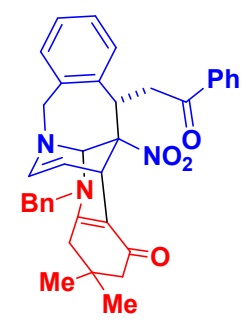

5-benzyl-3,3-dimethyl-12a-nitro-12-(2-oxo-2-phenylethyl)-3,4,5,5a,7,12,12a,13-octahydro-6,13-et henobenzo[5,6]azepino[2,3-b]quinolin-1(2H)-one (3z)

Yellow solid obtained by column chromatography (petroleum ether/ethyl acetate $=4: 1$ to $3: 1$ ); 6.9mg, 8\% yield; $\mathrm{dr}>20: 1$; reaction time $=2 \mathrm{~h} ; \mathrm{mp} 147.5-148.4{ }^{\circ} \mathrm{C} ;{ }^{1} \mathrm{H}$ NMR $\left(300 \mathrm{MHz}, \mathrm{CDCl}_{3}\right)$, $\delta 7.87(\mathrm{~d}, J=9.0 \mathrm{~Hz}, 2 \mathrm{H}), 7.51(\mathrm{t}, J=8.0 \mathrm{~Hz}, 1 \mathrm{H}), 7.40(\mathrm{t}, J=8.0 \mathrm{~Hz}, 2 \mathrm{H}), 7.23-7.19(\mathrm{~m}, 4 \mathrm{H})$, 7.07-6.95 (m, 5H), $6.76(\mathrm{~d}, J=8.0 \mathrm{~Hz}, 1 \mathrm{H}), 6.05(\mathrm{~d}, J=8.0 \mathrm{~Hz}, 1 \mathrm{H}), 5.13(\mathrm{t}, J=8.0 \mathrm{~Hz}, 1 \mathrm{H}), 4.62$ (s, 1H), 4.52-4.31 (m, 4H), $4.22(\mathrm{~d}, J=8.0 \mathrm{~Hz}, 1 \mathrm{H}), 3.60-3.45(\mathrm{~m}, 2 \mathrm{H}), 2.16-2.01(\mathrm{~m}, 4 \mathrm{H}), 0.83$ (s, 6H); ${ }^{13} \mathrm{C}$ NMR $\left(75 \mathrm{MHz}, \mathrm{CDCl}_{3}\right) \delta 196.1,192.0,156.8,137.0,136.5,136.2,135.2,133.7$, $133.5,128.7,128.0,127.6,127.5,127.5,126.5,125.7,107.8,106.7,88.5,71.5,58.6,52.1,49.1$, 39.6, 38.5, 35.1, 32.7, 29.0, 27.4, two carbons missing in the aromatic region. IR (KBr) $v 3295$, 2926, 1660, 1623, 1566, $739 \mathrm{~cm}^{-1}$. HRMS (ESI) calcd for $\mathrm{C}_{36} \mathrm{H}_{36} \mathrm{~N}_{3} \mathrm{O}_{4}[\mathrm{M}+\mathrm{H}]^{+}$574.2700, found 574.2698 .

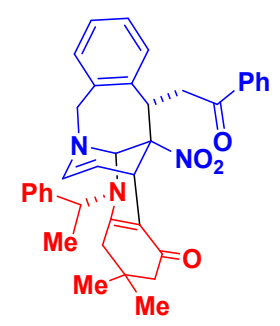

3,3-dimethyl-12a-nitro-12-(2-oxo-2-phenylethyl)-5-((R)-1-phenylethyl)-3,4,5,5a,7,12,12a,13-octa hydro-6,13-ethenobenzo[5,6]azepino[2,3-b]quinolin-1(2H)-one (3za)

Yellow solid obtained by column chromatography (petroleum ether/ethyl acetate $=4: 1$ ); $8.6 \mathrm{mg}$, $10 \%$ yield; $\mathrm{dr}=1.4: 1 ;$ reaction time $=2 \mathrm{~h} ; \mathrm{mp} 116.5-117.5^{\circ} \mathrm{C} ;{ }^{1} \mathrm{H}$ NMR $\left(300 \mathrm{MHz}, \mathrm{CDCl}_{3}\right), \delta 7.94$ $(\mathrm{d}, J=9.0 \mathrm{~Hz}, 2 \mathrm{H}), 7.58(\mathrm{t}, J=6.0 \mathrm{~Hz}, 1 \mathrm{H}), 7.45(\mathrm{t}, J=8.0 \mathrm{~Hz}, 2 \mathrm{H}), 7.38(\mathrm{t}, J=6.0 \mathrm{~Hz}, 1 \mathrm{H})$, 7.30-7.18 (m, 5H), 7.18-7.02 (m, 2H), 6.98-6.80 (m, 2H), $6.23(\mathrm{~d}, J=6.0 \mathrm{~Hz}, 1 \mathrm{H}), 5.23(\mathrm{t}, J=6.0$ $\mathrm{Hz}, 1 \mathrm{H}), 5.07-4.86(\mathrm{~m}, 1 \mathrm{H}), 4.83-4.59(\mathrm{~m}, 1 \mathrm{H}), 4.54-4.24(\mathrm{~m}, 3 \mathrm{H}), 4.19-3.96(\mathrm{~m}, 1 \mathrm{H}), 3.65-3.41$ 
(m, 2H), 2.40-2.13 (m, 3H), $1.65(\mathrm{~d}, J=6.0 \mathrm{~Hz}, 3 \mathrm{H}), 0.90(\mathrm{~s}, 3 \mathrm{H}), 0.84(\mathrm{~s}, 3 \mathrm{H}) ;{ }^{13} \mathrm{C}$ NMR $(75$ $\left.\mathrm{MHz}, \mathrm{CDCl}_{3}\right) \delta 196.2,191.9,156.9,139.9,137.3,136.2,135.3,133.4,128.6,128.2,127.9,127.8$, $127.6,127.5,127.4,127.0,125.3,108.2,107.1,88.8,58.0,57.2,49.0,40.1,38.3,35.1,32.2,29.6$, 28.9, 28.3, 27.5, 17.5. IR (KBr) v 3429, 2924, 1624, 1568, 1390, $743 \mathrm{~cm}^{-1}$. HRMS (ESI) calcd for $\mathrm{C}_{37} \mathrm{H}_{38} \mathrm{~N}_{3} \mathrm{O}_{4}[\mathrm{M}+\mathrm{H}]^{+}$588.2857, found 588.2856.

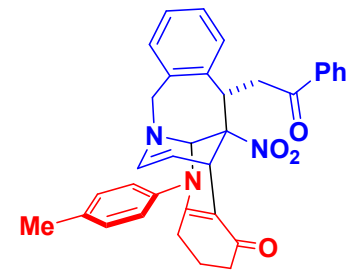

12a-nitro-12-(2-oxo-2-phenylethyl)-5-( $p$-tolyl)-3,4,5,5a,7,12,12a,13-octahydro-6,13-ethenobenzo[ 5,6]azepino[2,3-b]quinolin-1(2H)-one (3zb)

Yellow solid obtained by column chromatography (petroleum ether/ethyl acetate $=3: 1$ ); $42.0 \mathrm{mg}$, $51 \%$ yield; $\mathrm{dr}>20: 1 ;$ reaction time $=2 \mathrm{~h} ; \mathrm{mp} 215.2-215.9^{\circ} \mathrm{C} ;{ }^{1} \mathrm{H}$ NMR $\left(400 \mathrm{MHz}, \mathrm{CDCl}_{3}\right), \delta 7.96$ $(\mathrm{d}, J=8.0 \mathrm{~Hz}, 2 \mathrm{H}), 7.57(\mathrm{~d}, J=8.0 \mathrm{~Hz}, 1 \mathrm{H}), 7.47(\mathrm{~d}, J=8.0 \mathrm{~Hz}, 2 \mathrm{H}), 7.14-6.88(\mathrm{~m}, 8 \mathrm{H}), 6.16(\mathrm{~d}$, $J=4.0 \mathrm{~Hz}, 1 \mathrm{H}), 5.24(\mathrm{~s}, 1 \mathrm{H}), 5.02(\mathrm{~s}, 1 \mathrm{H}), 4.55(\mathrm{~s}, 1 \mathrm{H}), 4.45-4.35(\mathrm{~m}, 3 \mathrm{H}), 3.72-3.56(\mathrm{~m}, 2 \mathrm{H})$, $2.35(\mathrm{~s}, 3 \mathrm{H}), 2.25(\mathrm{~d}, J=4.0 \mathrm{~Hz}, 2 \mathrm{H}), 2.04(\mathrm{~d}, J=16.0 \mathrm{~Hz}, 1 \mathrm{H}), 1.89(\mathrm{~d}, J=16.0 \mathrm{~Hz}, 1 \mathrm{H}), 1.73(\mathrm{~s}$, 2H); ${ }^{13} \mathrm{C}$ NMR (100 MHz, $\left.\mathrm{CDCl}_{3}\right) \delta 196.1,192.7,157.7,139.3,138.1,137.5,136.3,135.1,133.7$, $133.5,130.4,130.2,129.9,128.7,128.1,128.0,127.6,127.4,125.7,108.3,88.9,73.8,58.5,38.5$, 35.6, 35.3, 29.3, 27.2, 21.4, 21.1. IR (KBr) $v$ 3430, 2948, 1624, 1571, 1387, 1130, $746 \mathrm{~cm}^{-1}$. HRMS (ESI) calcd for $\mathrm{C}_{34} \mathrm{H}_{32} \mathrm{~N}_{3} \mathrm{O}_{4}[\mathrm{M}+\mathrm{H}]^{+}$546.2387, found 546.2381 .

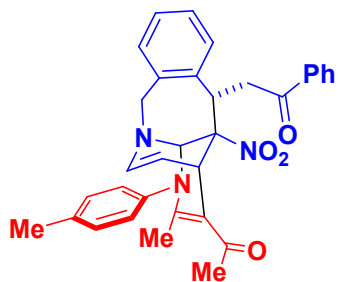

2-(3-acetyl-2-methyl-4a-nitro-1-( $p$-tolyl)-1,4,4a,5,10,11a-hexahydro-4,11-ethenobenzo[ $e]$ pyrido[2 ,3-b]azepin-5-yl)-1-phenylethan-1-one (3zc)

Yellow solid obtained by column chromatography (petroleum ether/ethyl acetate $=4: 1$ ); $25.6 \mathrm{mg}$, $32 \%$ yield; $\mathrm{dr}>20: 1 ;$ reaction time $=2 \mathrm{~h} ; \mathrm{mp} 127.4-128.3^{\circ} \mathrm{C} ;{ }^{1} \mathrm{H}$ NMR $\left(300 \mathrm{MHz}, \mathrm{CDCl}_{3}\right), \delta 8.00$ $(\mathrm{d}, J=6.0 \mathrm{~Hz}, 2 \mathrm{H}), 7.61(\mathrm{t}, J=8.0 \mathrm{~Hz}, 1 \mathrm{H}), 7.50(\mathrm{t}, J=8.0 \mathrm{~Hz}, 2 \mathrm{H}), 7.15-7.06(\mathrm{~m}, 4 \mathrm{H}), 6.94(\mathrm{dd}$, $\left.J_{l}=J_{2}=6.0 \mathrm{~Hz}, 4 \mathrm{H}\right), 6.21(\mathrm{~d}, J=8.0 \mathrm{~Hz}, 1 \mathrm{H}), 5.20(\mathrm{t}, J=8.0 \mathrm{~Hz}, 1 \mathrm{H}), 5.02(\mathrm{~s}, 1 \mathrm{H}), 4.47-4.22(\mathrm{~m}$, 
4H), $3.71\left(\mathrm{dd}, J_{1}=J_{2}=12.0 \mathrm{~Hz}, 1 \mathrm{H}\right), 3.51(\mathrm{~d}, J=18.0 \mathrm{~Hz}, 1 \mathrm{H}), 2.36(\mathrm{~s}, 3 \mathrm{H}), 2.33(\mathrm{~s}, 3 \mathrm{H}), 1.93(\mathrm{~s}$, 3H); ${ }^{13} \mathrm{C}$ NMR (75 MHz, $\left.\mathrm{CDCl}_{3}\right) \delta$ 196.3, 194.1, 153.3, 140.1, 137.7, 137.5, 136.4, 135.2, 133.6, 133.4, 130.4, 129.7, 129.5, 128.8, 128.1, 127.8, 127.4, 125.7, 107.9, 107.5, 89.4, 73.3, 57.9, 38.6, 35.4, 34.4, 30.0, 21.1, 19.0. IR (KBr) v 3434, 2923, 1631, 1547, 1138, $754 \mathrm{~cm}^{-1}$. HRMS (ESI) calcd for $\mathrm{C}_{33} \mathrm{H}_{32} \mathrm{~N}_{3} \mathrm{O}_{4}[\mathrm{M}+\mathrm{H}]^{+}$534.2387, found 534.2388.

\section{Chemical transformations of $3 a$}

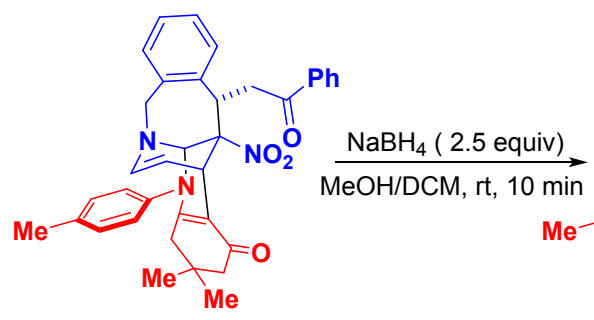

$3 a$

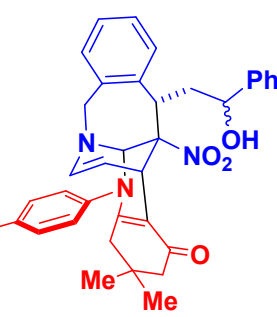

4/4', 99\% (1:1)

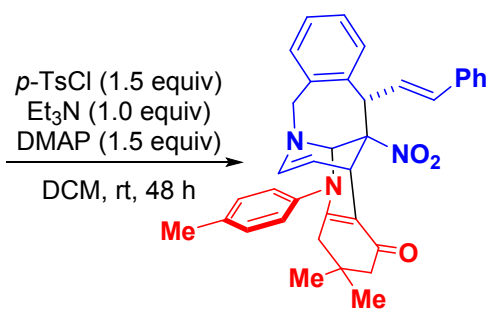

5, $23 \%$

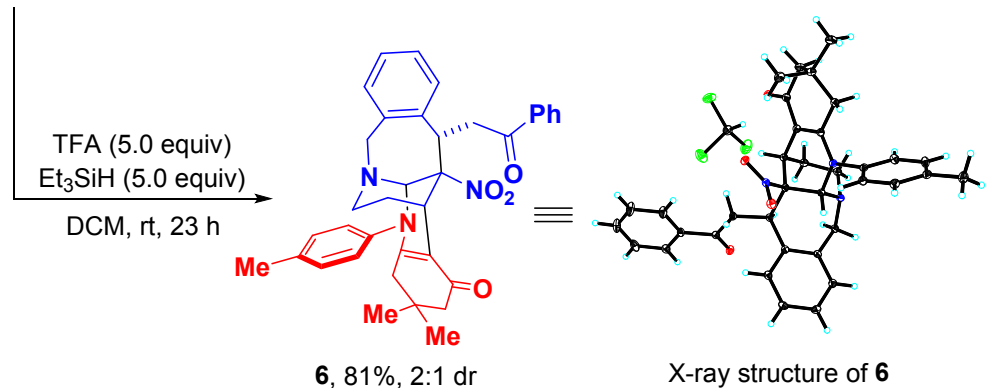

General procedure for the formation of 4/4': A solution of $\mathbf{3 a}(229.5 \mathrm{mg}, 0.40 \mathrm{mmol})$ in 40 $\mathrm{mL} \mathrm{MeOH}$ and $2.0 \mathrm{~mL}$ DCM was cooled to $0{ }^{\circ} \mathrm{C}$, and then $\mathrm{NaBH}_{4}(37.8 \mathrm{mg}, 1.0 \mathrm{mmol})$ was added successively. The reaction mixture was stirred at $0^{\circ} \mathrm{C}$ for $10 \mathrm{~min}$ until the complete consumption of 3a as monitored by thin layer chromatography. Then, saturated aq. $\mathrm{NH}_{4} \mathrm{Cl}$ solution was added. The mixture was extracted with $\mathrm{CH}_{2} \mathrm{Cl}_{2}$. The combined organic phase was dried over $\mathrm{MgSO}_{4}$, filtered, concentrated and purified with silica gel column chromatography to obtain $\mathbf{4} / \mathbf{4}^{\prime}$ ' in $99 \%$ yield with 1:1 dr.

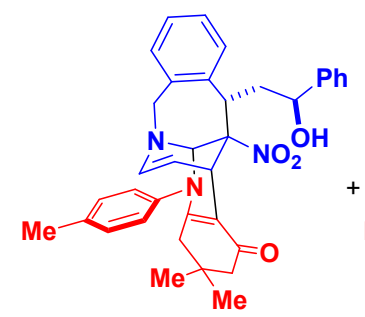

4

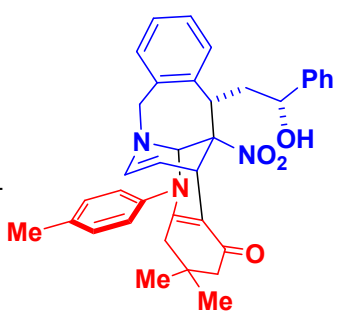

4

12-(2-hydroxy-2-phenylethyl)-3,3-dimethyl-12a-nitro-5-( $p$-tolyl)-3,4,5,5a,7,12,12a,13-octahydro6,13-ethenobenzo[5,6]azepino[2,3-b]quinolin-1(2H)-one (4/4') 
White solid obtained by silica gel column chromatography (petroleum ether/ethylacetate $=4: 1$ to 1:1), 227.5mg, 99\% yield; Reaction time $=10 \mathrm{~min} ; \mathrm{dr}=1: 1$ (4/4', separable isomers); $\mathrm{m} . \mathrm{p}$. 231.8-232.4 ${ }^{\circ} \mathrm{C}(4), 233.1-233.7^{\circ} \mathrm{C}\left(\mathbf{4}^{\prime}\right) ;{ }^{1} \mathrm{H}$ NMR (400 MHz, $\left.\mathrm{CDCl}_{3}\right)$ for 4, $\delta$ 7.34-7.18 (m, 7H), $7.08(\mathrm{q}, J=8.0 \mathrm{~Hz}, 4 \mathrm{H}), 6.93(\mathrm{~d}, J=4.0 \mathrm{~Hz}, 1 \mathrm{H}), 6.84(\mathrm{br}, 2 \mathrm{H}), 5.59$ (d, $J=8.0 \mathrm{~Hz}, 1 \mathrm{H}), 4.79$ (d, $J=8.0 \mathrm{~Hz}, 2 \mathrm{H}), 4.66(\mathrm{~s}, 1 \mathrm{H}), 4.43(\mathrm{~d}, J=4.0 \mathrm{~Hz}, 1 \mathrm{H}), 4.15(\mathrm{~d}, J=16.0 \mathrm{~Hz}, 1 \mathrm{H}), 3.78(\mathrm{~d}, J=12.0$ $\mathrm{Hz}, 1 \mathrm{H}), 3.11-2.97(\mathrm{~m}, 2 \mathrm{H}), 2.45-2.22(\mathrm{~m}, 6 \mathrm{H}), 1.83(\mathrm{~d}, J=16.0 \mathrm{~Hz}, 1 \mathrm{H}), 1.63(\mathrm{~d}, J=16.0 \mathrm{~Hz}$, 1H), $0.80(\mathrm{~s}, 3 \mathrm{H}), 0.75(\mathrm{~s}, 3 \mathrm{H}) ;{ }^{13} \mathrm{C} \mathrm{NMR}\left(100 \mathrm{MHz}, \mathrm{CDCl}_{3}\right)$ for $4 \delta 192.3,156.3,143.3,139.3$, 139.3, 137.8, 137.6, 135.0, 132.9, 128.5, 127.8, 127.7, 127.6, 127.5, 127.4, 126.1, 108.0, 107.1, $88.3,74.0,72.5,58.5,49.3,40.7,39.8,34.8,32.6,28.7,28.6,27.4,21.1 .{ }^{1} \mathrm{H}$ NMR (400 MHz, $\mathrm{CDCl}_{3}$ ) for 4', $\delta$ 7.37-7.29 (m, 5H), 7.24-7.14 (m, 5H), 7.03-6.92 (m, 3H), $6.13(\mathrm{~d}, J=4.0 \mathrm{~Hz}$, $1 \mathrm{H}), 5.31(\mathrm{t}, J=8.0 \mathrm{~Hz}, 1 \mathrm{H}), 4.99(\mathrm{~d}, J=4.0 \mathrm{~Hz}, 1 \mathrm{H}), 4.78(\mathrm{~d}, J=4.0 \mathrm{~Hz}, 1 \mathrm{H}), 4.46(\mathrm{~d}, J=12.0$ Hz, 1H), 4.34 (q, $J=16.0 \mathrm{~Hz}, 2 \mathrm{H}), 4.13$ (d, $J=12.0 \mathrm{~Hz}, 1 \mathrm{H}), 3.44$ (s, 1H), 2.56 (t, $J=12.0 \mathrm{~Hz}$, 1H), 2.37 (s, 3H), 2.05 (t, $J=12.0 \mathrm{~Hz}, 3 \mathrm{H}), 1.89$ (d, $J=16.0 \mathrm{~Hz}, 1 \mathrm{H}), 1.67(\mathrm{~d}, J=16.0 \mathrm{~Hz}, 1 \mathrm{H})$, 0.82 (s, 3H), 0.74 (s, 3H); ${ }^{13} \mathrm{C}$ NMR (100 MHz, $\mathrm{CDCl}_{3}$ ) for 4' $\delta$ 192.6, 156.6, 144.8, 139.3, 138.5, $137.9,134.9,133.4,129.8,128.5,128.2,127.6,127.5,127.3,127.0,125.7, .108 .7,107.4,89.0$, 74.5, 71.0, 58.6, 49.1, 40.8, 39.7, 34.8, 32.6, 28.8, 28.7, 27.3, $21.1 \mathrm{IR}(\mathrm{KBr})$ for $\mathbf{4} v 3402,2955$, $1620,1569,1391,762 \mathrm{~cm}^{-1}$. IR (KBr) for 4'v 3437, 2948, 1624, 1570, 1395, 752cm-1. HRMS (ESI) calcd for $\mathrm{C}_{36} \mathrm{H}_{38} \mathrm{~N}_{3} \mathrm{O}_{4}[\mathrm{M}+\mathrm{H}]^{+}$576.2857, found 576.2845.

General procedure for the formation of 5: To a solution of 4/4' $(221.1 \mathrm{mg}, 0.38 \mathrm{mmol})$ in $2.0 \mathrm{~mL}$ DCM, $p$-TsCl (109.8 mg, 0.58mmol), DMAP (46.9 mg, $0.38 \mathrm{mmol})$ and $\mathrm{Et}_{3} \mathrm{~N}(58.3 \mathrm{mg}$, $0.58 \mathrm{mmol}$ ) were successively added. The resulting mixture was stirred at room temperature for 48 h, then diluted with $\mathrm{Et}_{2} \mathrm{O}$, washed with saturated aq. $\mathrm{NaHCO}_{3}$ and brine, dried over $\mathrm{MgSO}_{4}$, filtered, concentrated and purified by silica gel column chromatography (petroleum ether/ ethyl acetate $=3: 1$ to $1: 1)$ to afford 5 as a yellow solid in $23 \%$ yield .

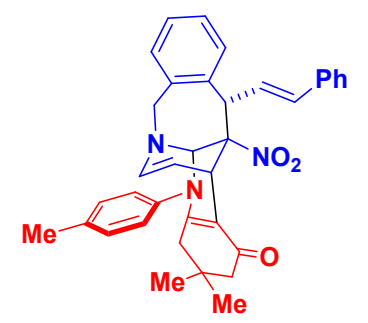

3,3-dimethyl-12a-nitro-12-((E)-styryl)-5-( $p$-tolyl)-3,4,5,5a,7,12,12a,13-octahydro-6,13-ethenoben zo[5,6]azepino[2,3-b]quinolin-1(2H)-one (5) 
Yellow solid obtained by column chromatography (petroleum ether/ethyl acetate $=3: 1$ to $1: 1$ ); $50.1 \mathrm{mg}, 23 \%$ yield; $\mathrm{dr}>20: 1$; reaction time $=48 \mathrm{~h} ; \mathrm{mp} 147.2-147.9^{\circ} \mathrm{C} ;{ }^{1} \mathrm{H}$ NMR $(400 \mathrm{MHz}$, $\left.\mathrm{CDCl}_{3}\right), \delta 7.31(\mathrm{~d}, J=4.0 \mathrm{~Hz}, 2 \mathrm{H}), 7.22(\mathrm{t}, J=8.0 \mathrm{~Hz}, 2 \mathrm{H}), 7.18-7.15(\mathrm{~m}, 3 \mathrm{H}), 7.12-7.07(\mathrm{~m}, 4 \mathrm{H})$, $6.92(\mathrm{~d}, J=8.0 \mathrm{~Hz}, 2 \mathrm{H}), 6.87(\mathrm{~d}, J=8.0 \mathrm{~Hz}, 1 \mathrm{H}), 6.52(\mathrm{~d}, J=16.0 \mathrm{~Hz}, 1 \mathrm{H}), 6.15\left(\mathrm{dd}, J_{l}=8.0 \mathrm{~Hz}\right.$, $\left.J_{2}=12.0 \mathrm{~Hz}, 1 \mathrm{H}\right), 5.94(\mathrm{~d}, J=8.0 \mathrm{~Hz}, 1 \mathrm{H}), 5.18(\mathrm{t}, J=8.0 \mathrm{~Hz}, 1 \mathrm{H}), 5.15(\mathrm{~d}, J=4.0 \mathrm{~Hz}, 1 \mathrm{H}), 4.36$ $(\mathrm{d}, J=4.0 \mathrm{~Hz}, 1 \mathrm{H}), 4.29$ (d, $J=8.0 \mathrm{~Hz}, 1 \mathrm{H}), 4.23(\mathrm{~d}, J=4.0 \mathrm{~Hz}, 1 \mathrm{H}), 2.30$ (s, 3H), 2.03 (t, $J=$ $16.0 \mathrm{~Hz}, 2 \mathrm{H}), 1.83(\mathrm{~d}, J=16.0 \mathrm{~Hz}, 1 \mathrm{H}), 1.65(\mathrm{~d}, J=16.0 \mathrm{~Hz}, 1 \mathrm{H}), 0.78(\mathrm{~s}, 3 \mathrm{H}), 0.73(\mathrm{~s}, 3 \mathrm{H}) ;{ }^{13} \mathrm{C}$ NMR $\left(100 \mathrm{MHz}, \mathrm{CDCl}_{3}\right) \delta 192.1,155.9,139.4,138.0,137.4,136.9,136.3,136.0,133.4,130.4$, 128.9, 128.5, 128.0, 127.8, 127.5, 127.4, 126.8, 122.5, 109.2, 107.9, 89.0, 74.6, 58.3, 49.4, 48.9, 40.8, 32.6, 29.6, 28.7, 27.4, 21.1. IR (KBr) v 3437, 2926, 2862, 1627, 1573, 1390, 807 $\mathrm{cm}^{-1}$. HRMS (ESI) calcd for $\mathrm{C}_{36} \mathrm{H}_{36} \mathrm{~N}_{3} \mathrm{O}_{3}[\mathrm{M}+\mathrm{H}]^{+}$558.2751, found 558.2758.

General procedure for the formation of 6: A solution of $\mathbf{3 a}(229.5 \mathrm{mg}, 0.40 \mathrm{mmol})$ in 2.0 $\mathrm{mL}$ DCM was cooled to $0{ }^{\circ} \mathrm{C}$, and then $\mathrm{Et}_{3} \mathrm{SiH}(232.6 \mathrm{mg}, 2.0 \mathrm{mmol})$ and TFA (46.9 mg, 2.0 mmol) was added successively. The reaction mixture was stirred at room temperature for $23 \mathrm{~h}$ until the complete consumption of $\mathbf{3 a}$ as monitored by thin layer chromatography. After completion of the reaction, the reaction mixture was concentrated and purified by silica gel column chromatography (petroleum ether/ ethyl acetate $=4: 1$ ) to afford $\mathbf{6}$ as a yellow solid in $81 \%$ yield with 2:1 dr.

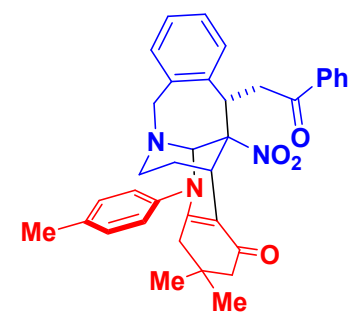

3,3-dimethyl-12a-nitro-12-(2-oxo-2-phenylethyl)-5-(p-tolyl)-3,4,5,5a,7,12,12a,13-octahydro-6,13ethanobenzo[5,6]azepino[2,3-b]quinolin-1(2H)-one (6)

Yellow solid obtained by column chromatography (petroleum ether/ethyl acetate $=4: 1$ ); 186.3 $\mathrm{mg}, 81 \%$ yield; $\mathrm{dr}=2: 1$ (inseperable isomers); reaction time $=23 \mathrm{~h} ; \mathrm{mp} 176.3-177.1^{\circ} \mathrm{C} ;{ }^{1} \mathrm{H} \mathrm{NMR}$ (400 MHz, DMSO- $\left.d_{6}\right), \delta 7.99(\mathrm{~d}, J=8.0 \mathrm{~Hz}, 1 \mathrm{H}), 7.90(\mathrm{~d}, J=8.0 \mathrm{~Hz}, 2 \mathrm{H}), 7.56$ (t, $J=8.0 \mathrm{~Hz}$, 2H), $7.41(\mathrm{t}, J=8.0 \mathrm{~Hz}, 3 \mathrm{H}), 7.29(\mathrm{~d}, J=4.0 \mathrm{~Hz}, 1 \mathrm{H}), 7.19-7.16(\mathrm{~m}, 4 \mathrm{H}), 5.56(\mathrm{~d}, J=4.0 \mathrm{~Hz}, 1 \mathrm{H})$, $4.59(\mathrm{~d}, J=16.0 \mathrm{~Hz}, 1 \mathrm{H}), 4.40\left(\mathrm{dd}, J_{1}=J_{2}=4.0 \mathrm{~Hz}, 1 \mathrm{H}\right), 4.23-4.15(\mathrm{~m}, 1 \mathrm{H}), 3.86(\mathrm{~s}, 1 \mathrm{H}), 3.76(\mathrm{t}$, $J=8.0 \mathrm{~Hz}, 1 \mathrm{H}), 3.09\left(\mathrm{dd}, J_{1}=J_{2}=4.0 \mathrm{~Hz}, 1 \mathrm{H}\right), 2.88-2.82(\mathrm{~m}, 1 \mathrm{H}), 2.48-2.44(\mathrm{~m}, 1 \mathrm{H}), 2.39(\mathrm{~s}$, 
3H), $2.26(\mathrm{~s}, 1 \mathrm{H}), 2.15(\mathrm{t}, J=16.0 \mathrm{~Hz}, 2 \mathrm{H}), 2.04(\mathrm{~d}, J=16.0 \mathrm{~Hz}, 1 \mathrm{H}), 1.92(\mathrm{~d}, J=16.0 \mathrm{~Hz}, 1 \mathrm{H})$, $1.72(\mathrm{~d}, J=16.0 \mathrm{~Hz}, 1 \mathrm{H}), 0.82(\mathrm{~s}, 3 \mathrm{H}), 0.72(\mathrm{~s}, 3 \mathrm{H}) ;{ }^{13} \mathrm{C}$ NMR $\left(100 \mathrm{MHz}, \mathrm{DMSO}-d_{6}\right) \delta 196.1$, $190.7,156.1,140.2,139.8,138.8,137.3,136.0,133.5,130.9,130.4,128.7,128.5,128.1,127.8$, 127.6, 107.3, 90.5, 76.3, 56.6, 54.9, 49.0, 48.3, 40.5, 36.1, 35.4, 32.4, 28.9, 28.8, 26.5, 20.7. IR $(\mathrm{KBr}) v 3443,2967,1687,1581,1409,1260,751 \mathrm{~cm}^{-1}$. HRMS (ESI) calcd for $\mathrm{C}_{36} \mathrm{H}_{38} \mathrm{~N}_{3} \mathrm{O}_{4}$ $[\mathrm{M}+\mathrm{H}]^{+} 576.2857$, found 576.2859 .

\section{Crystal data for $3 a, 3 \mathrm{~m}, 5$ and 6}

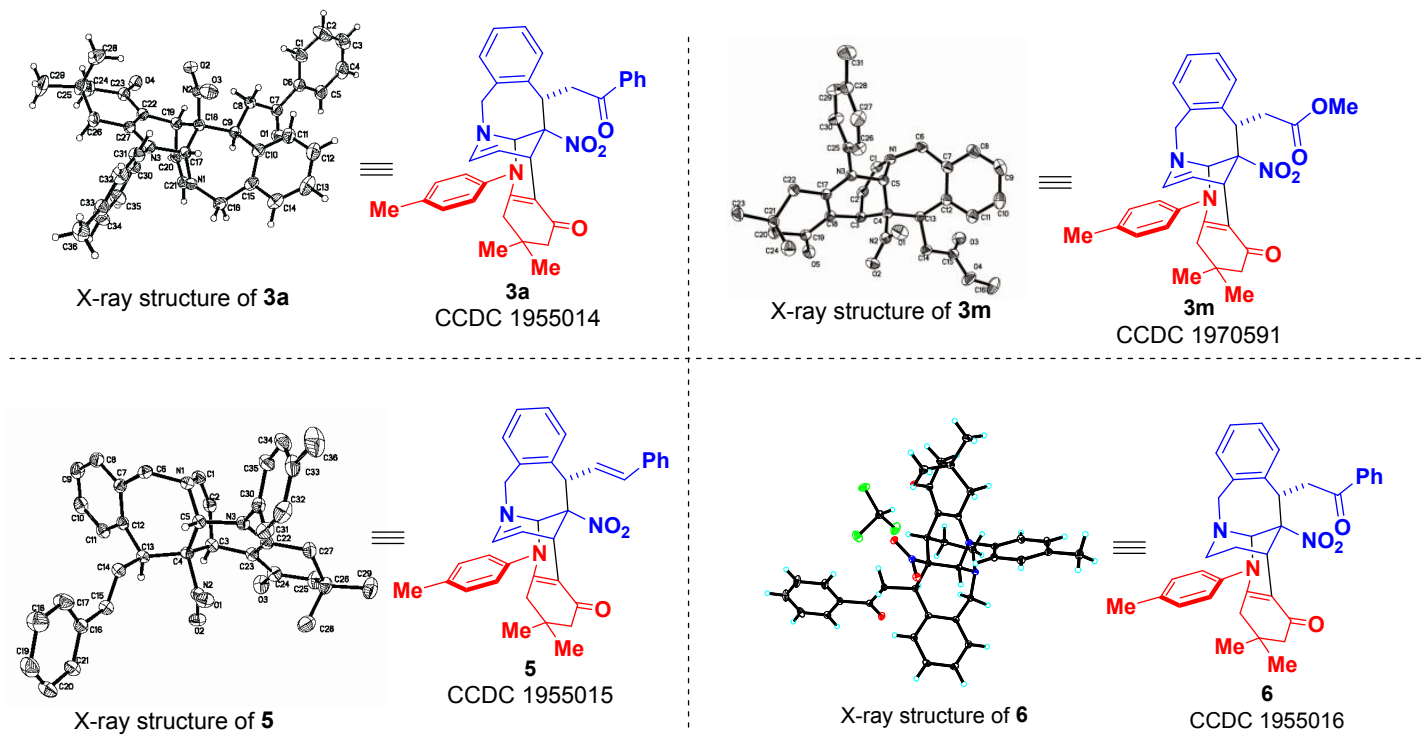

Displacement ellipsoids are drawn at the $30 \%$ probability level. 


\section{6. ${ }^{1} \mathrm{H}$ NMR and ${ }^{13} \mathrm{C}$ NMR spectra}

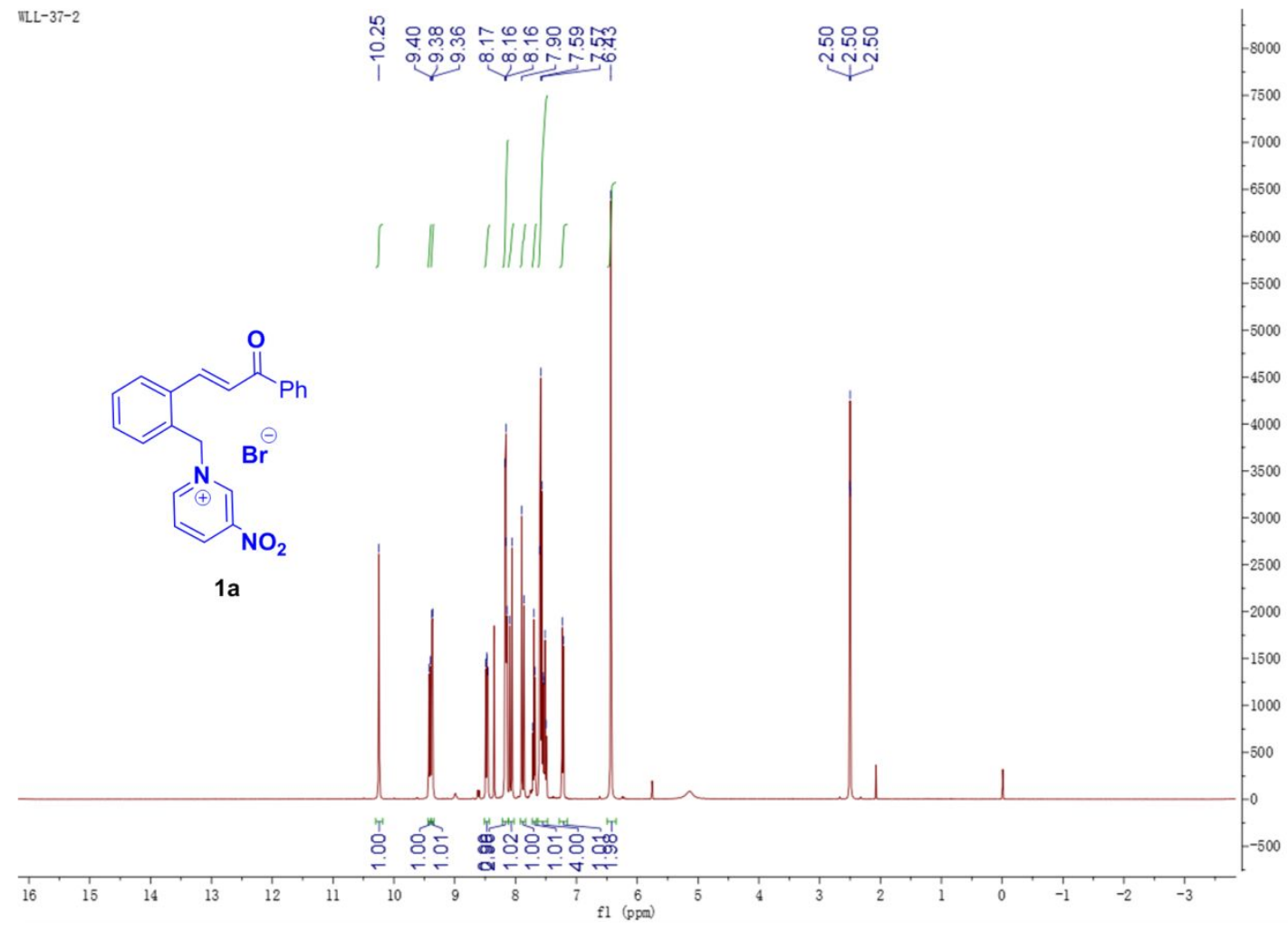



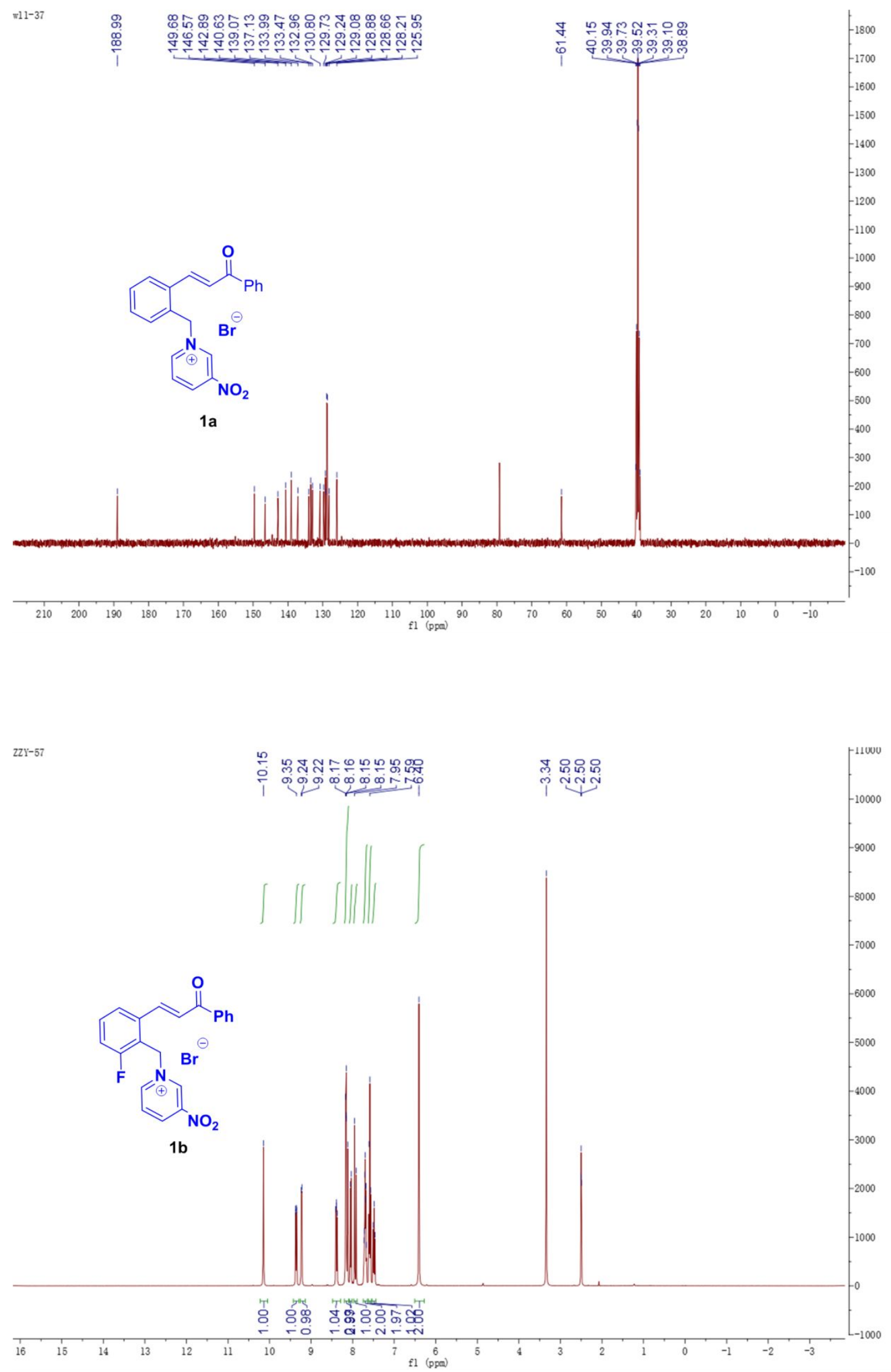

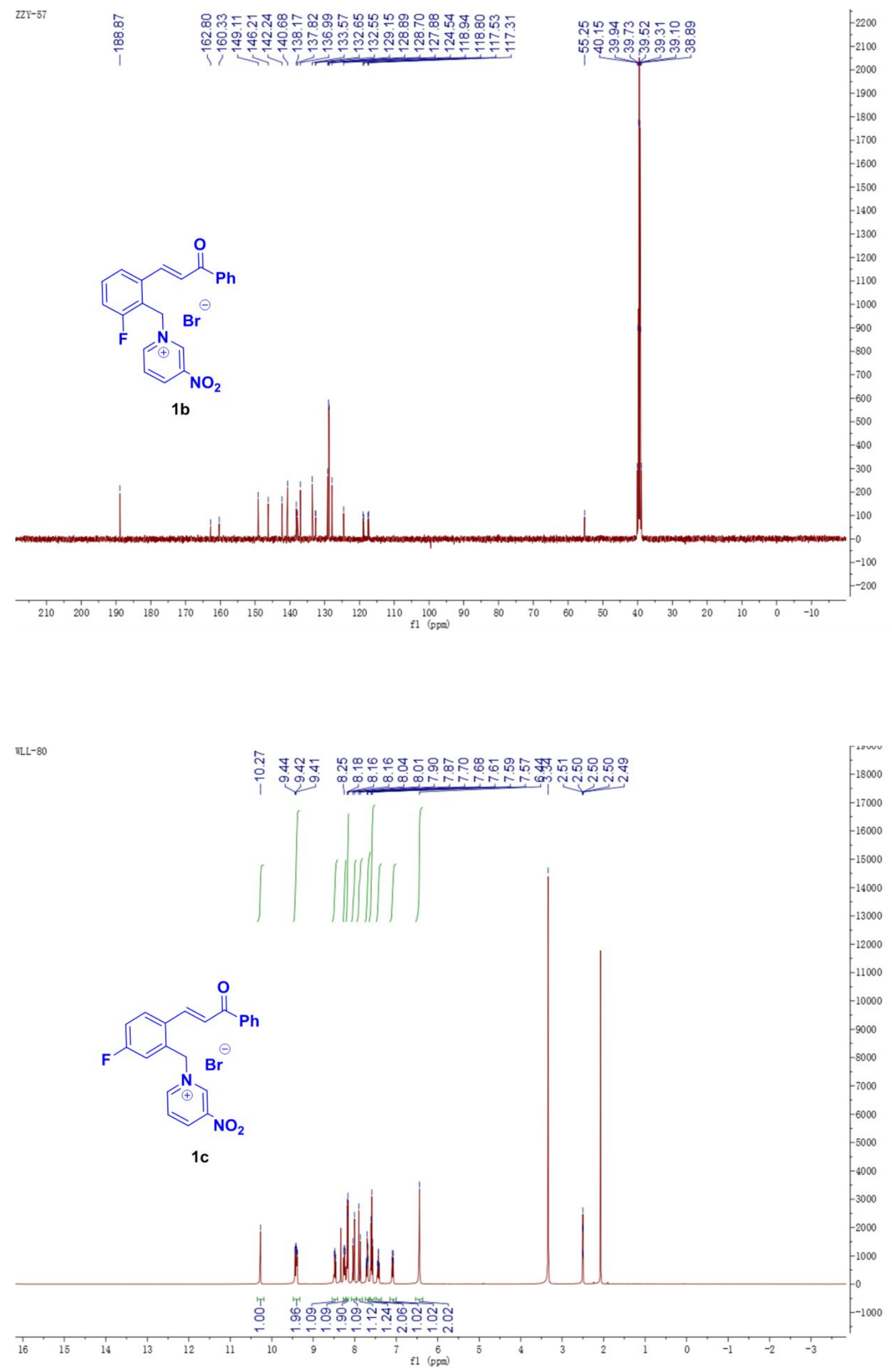

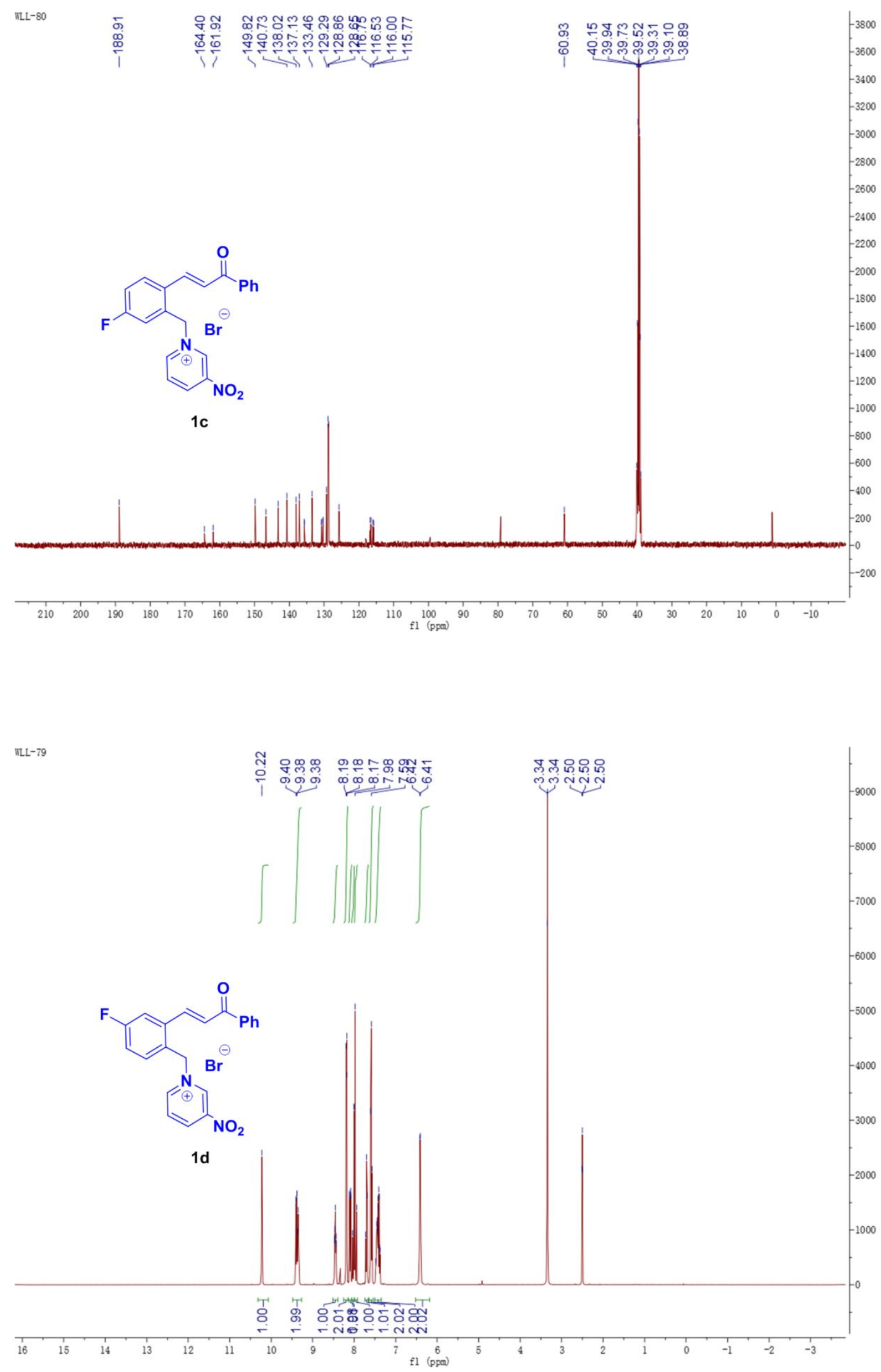

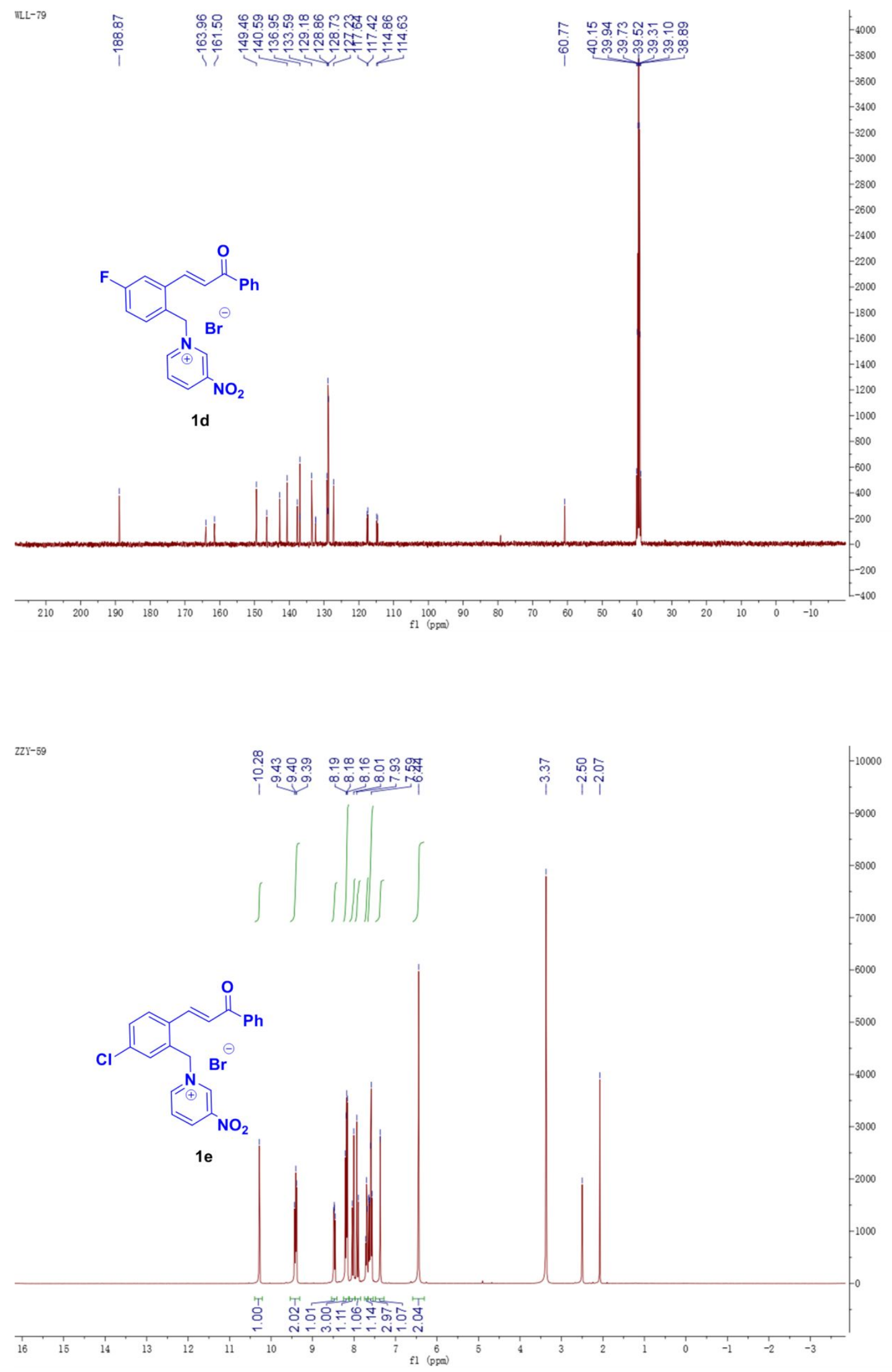

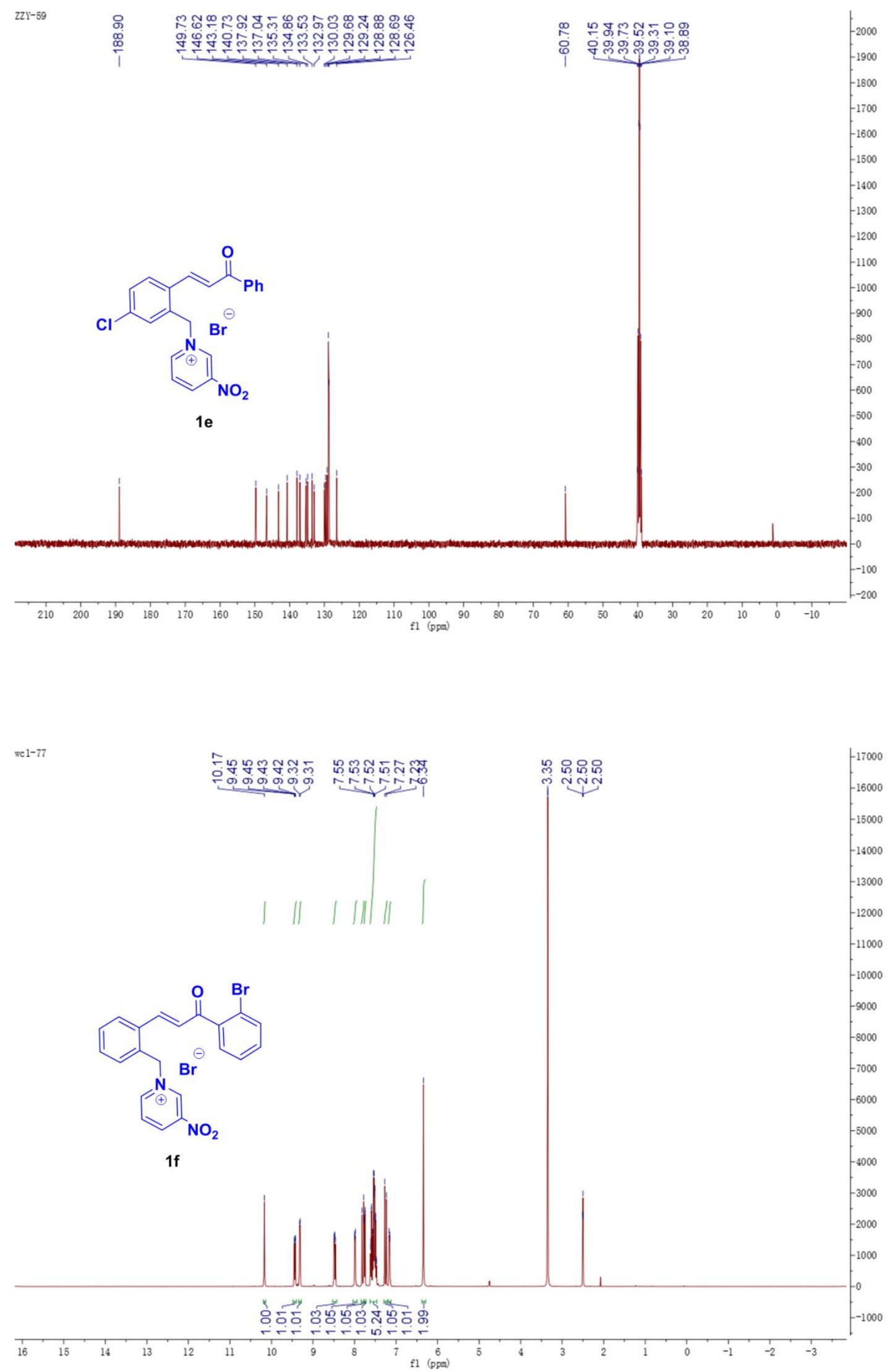

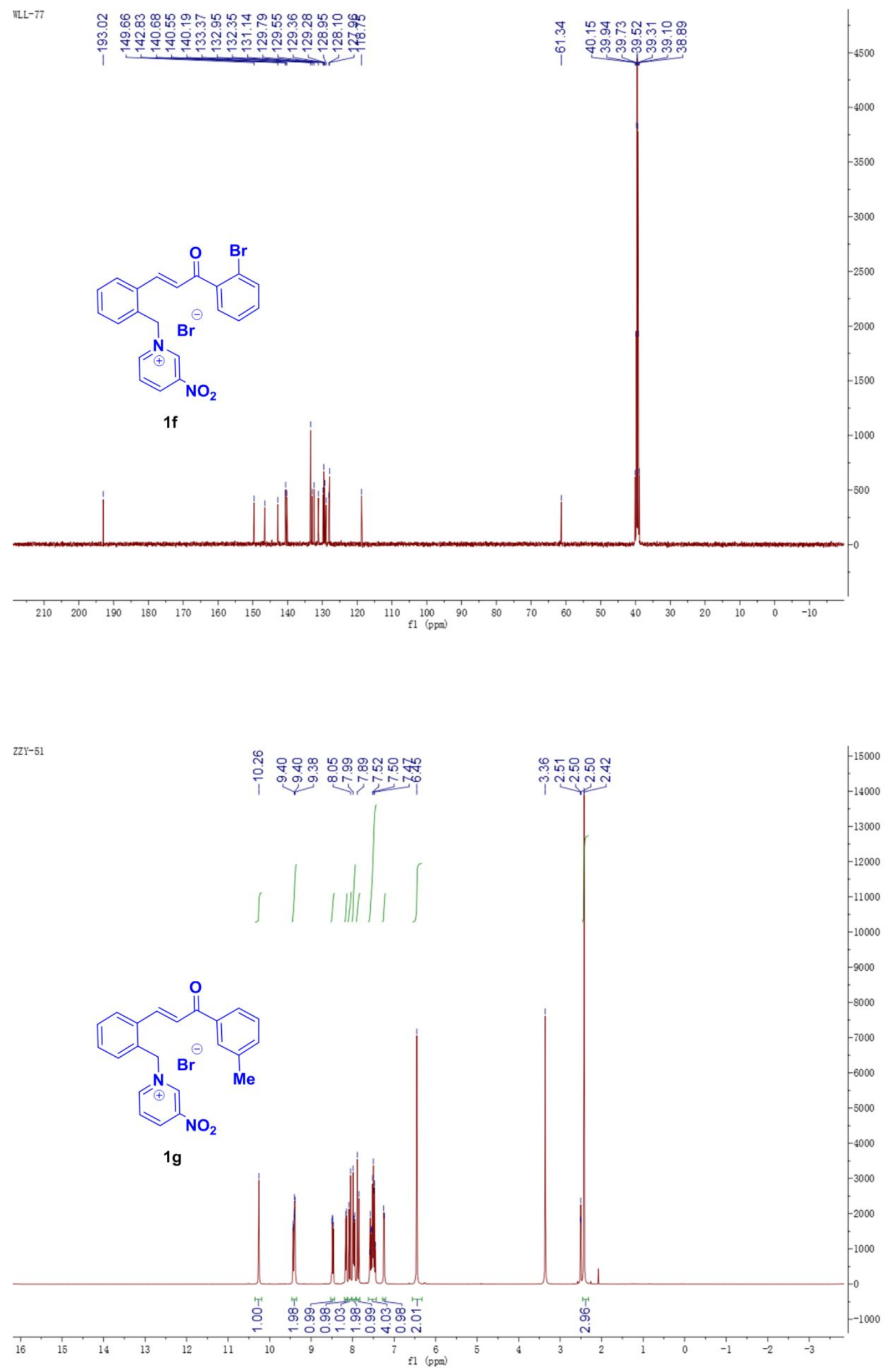

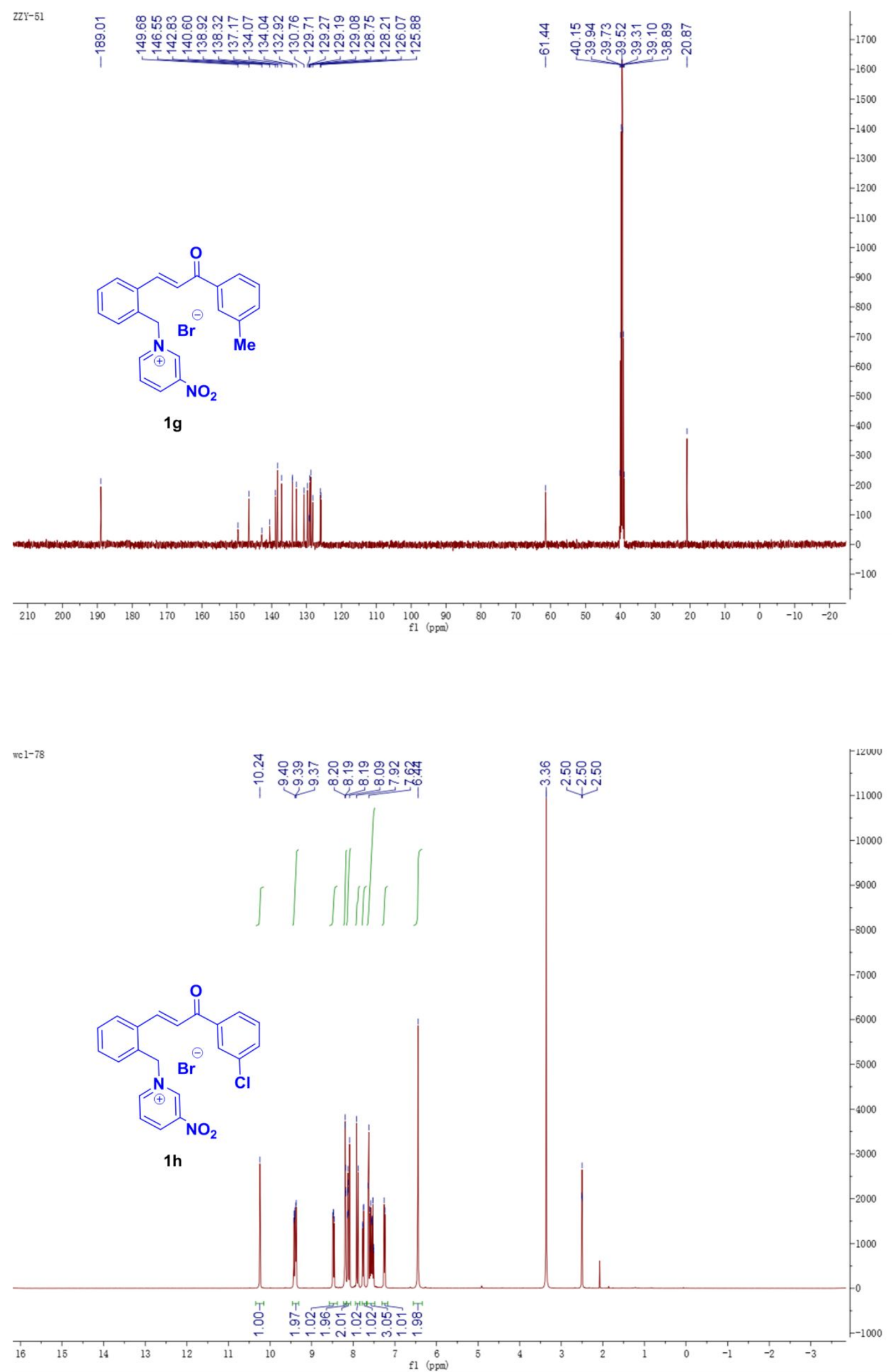

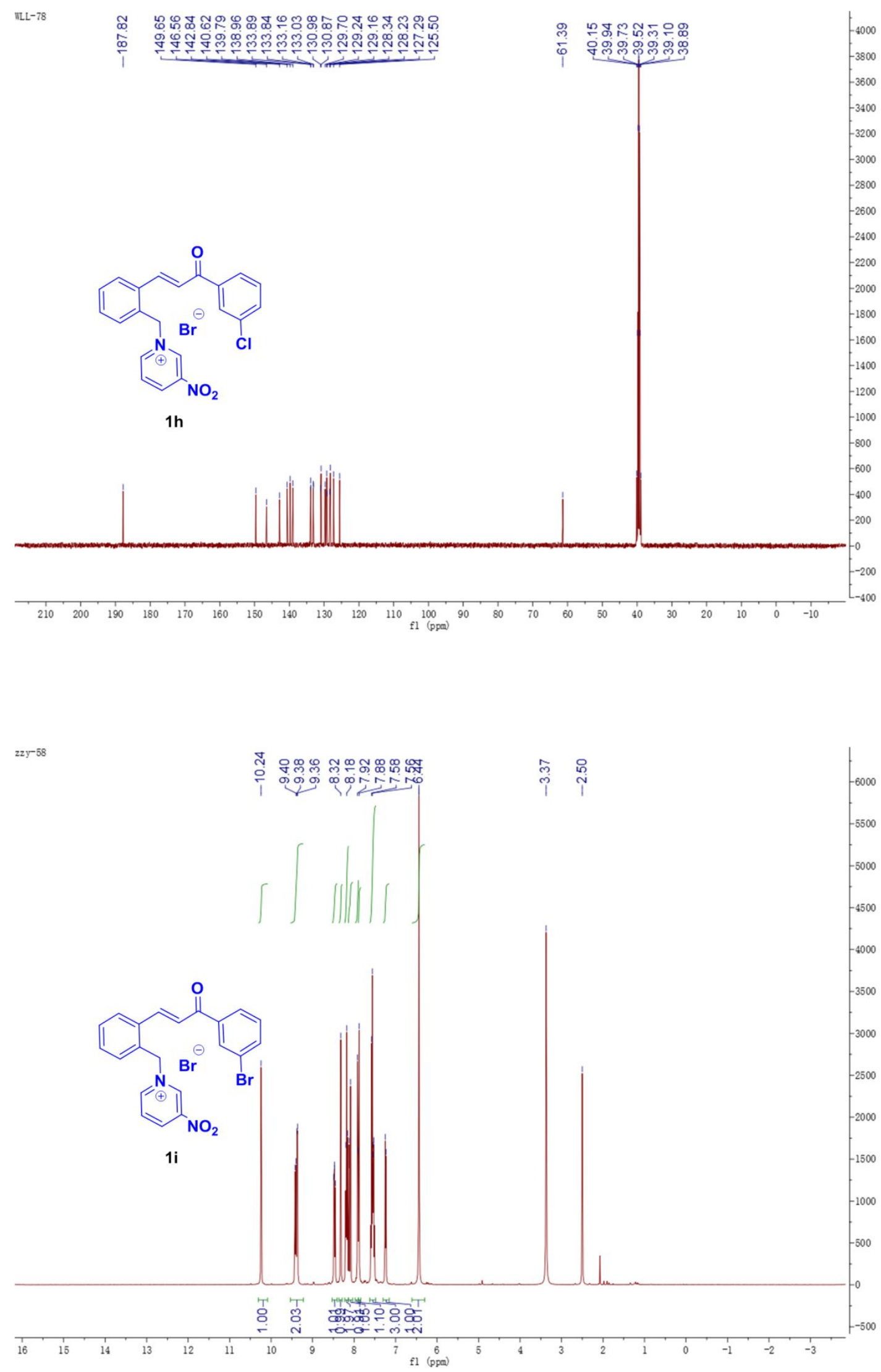

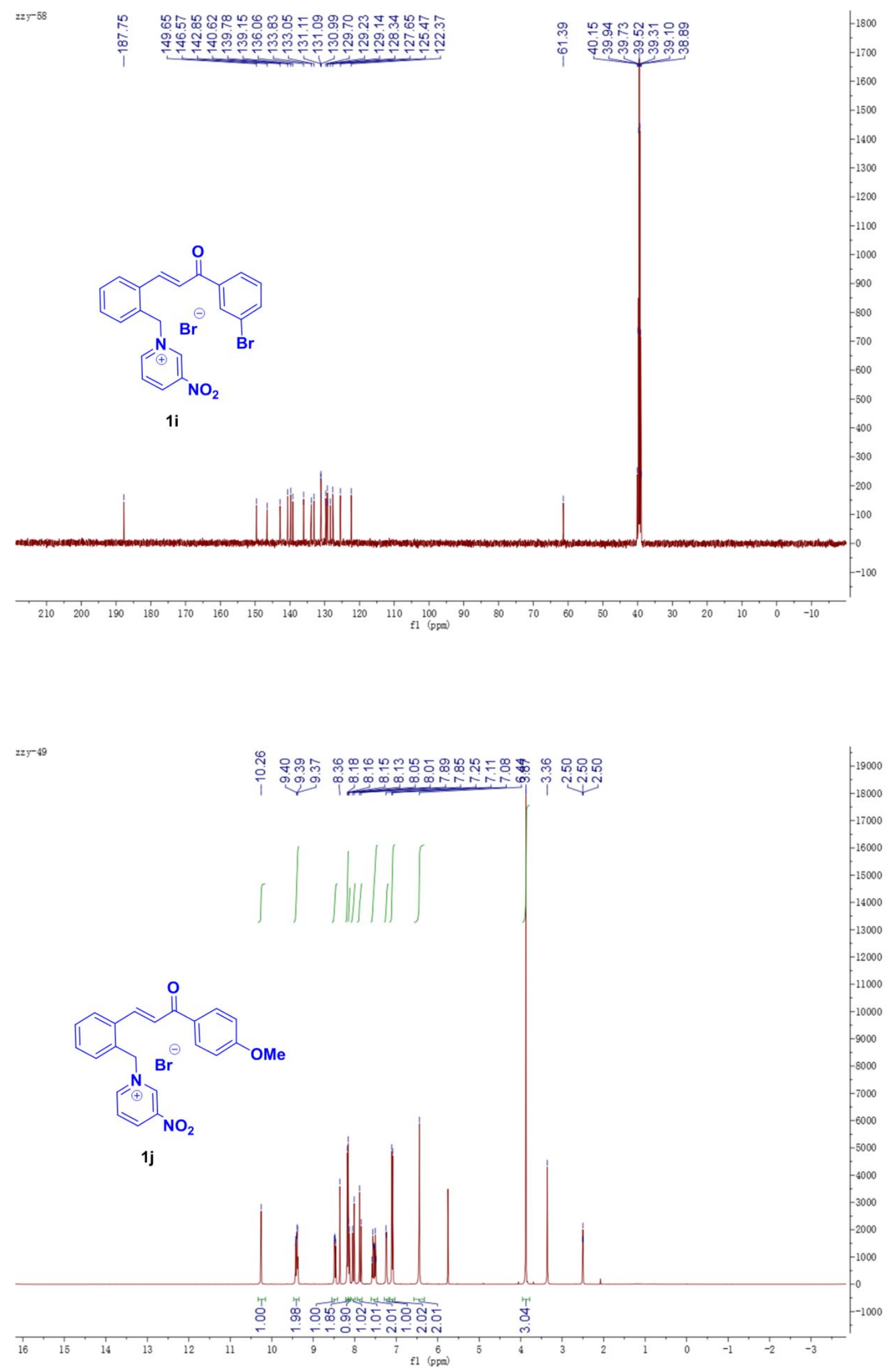

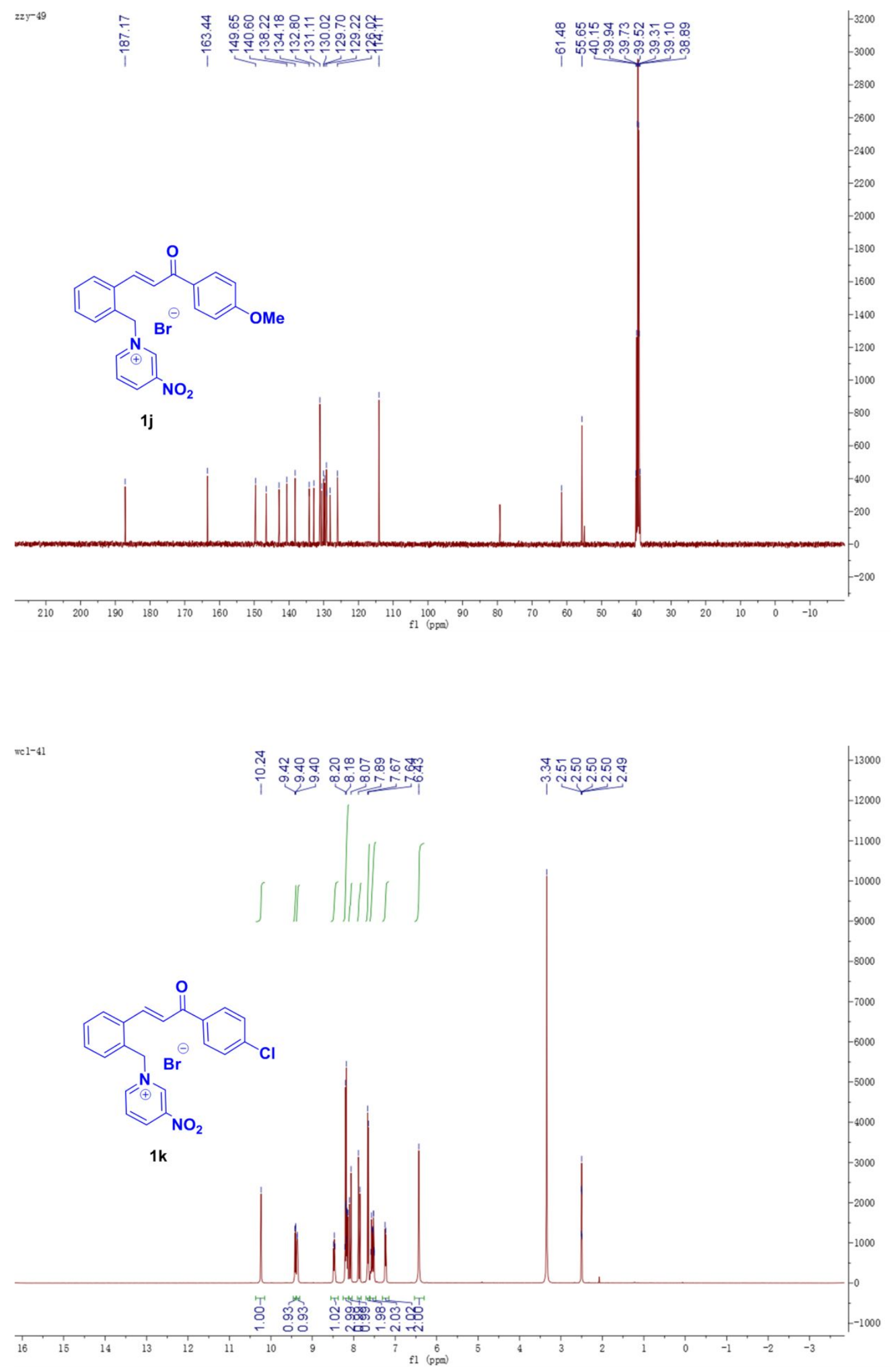

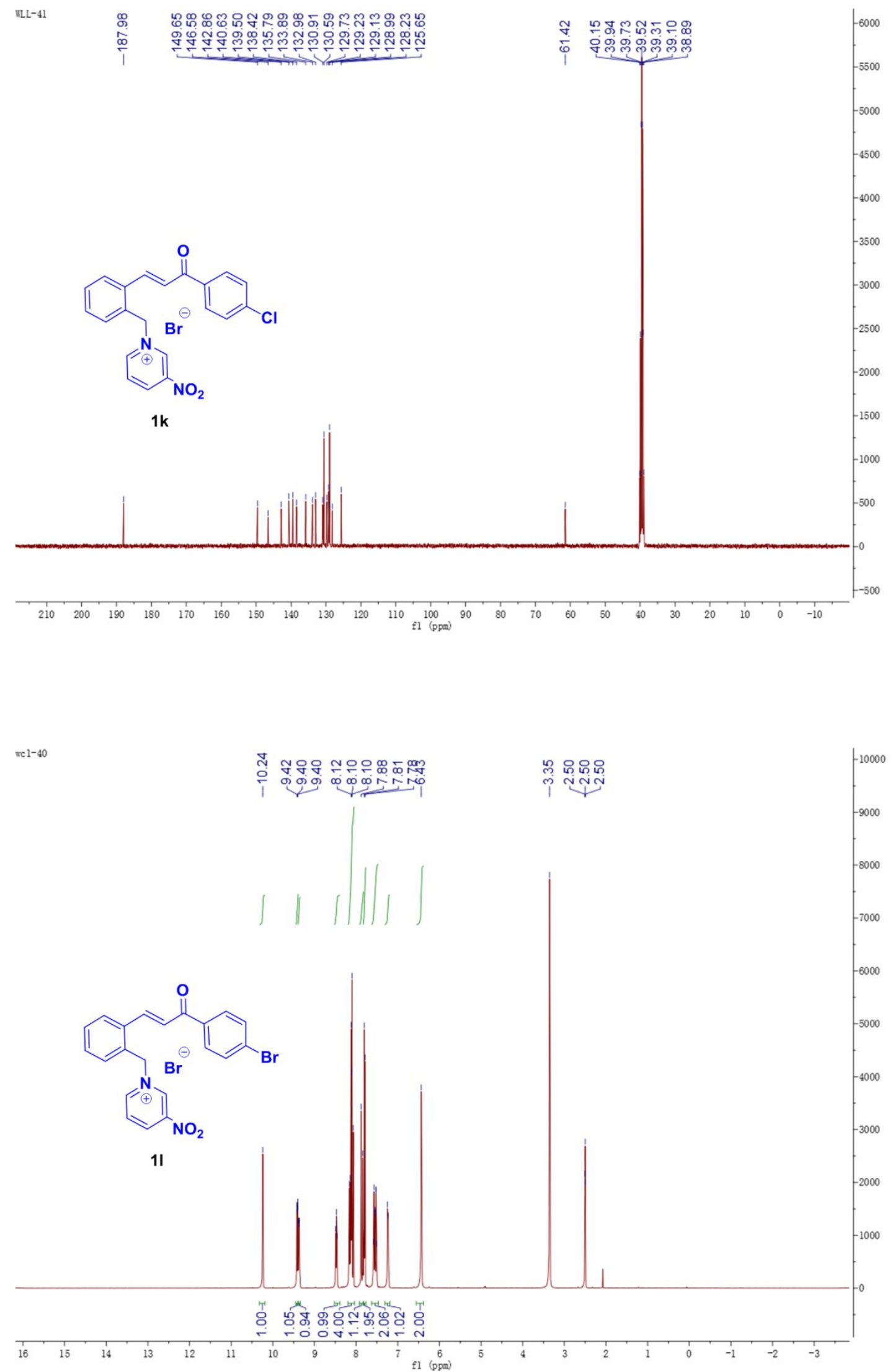

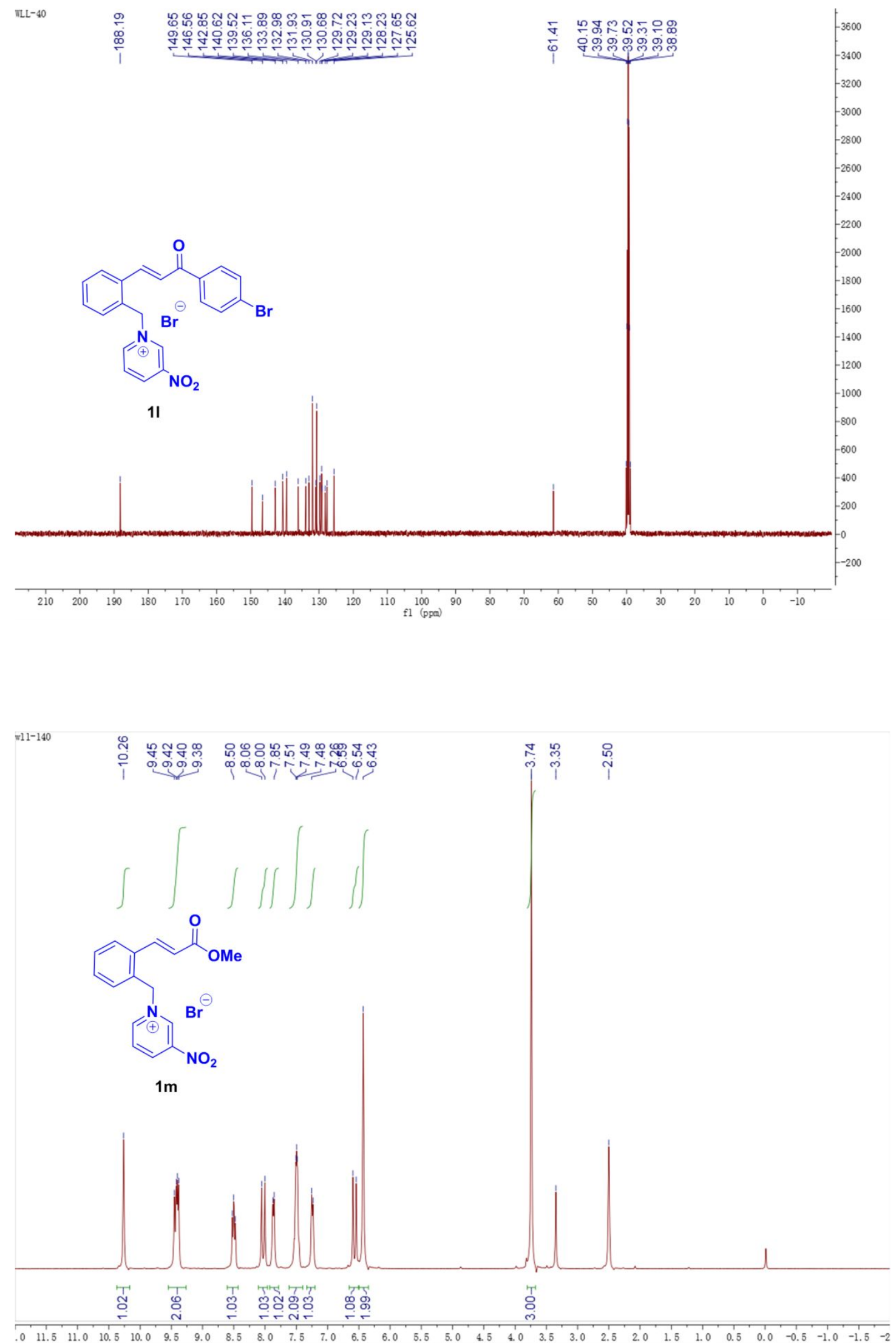

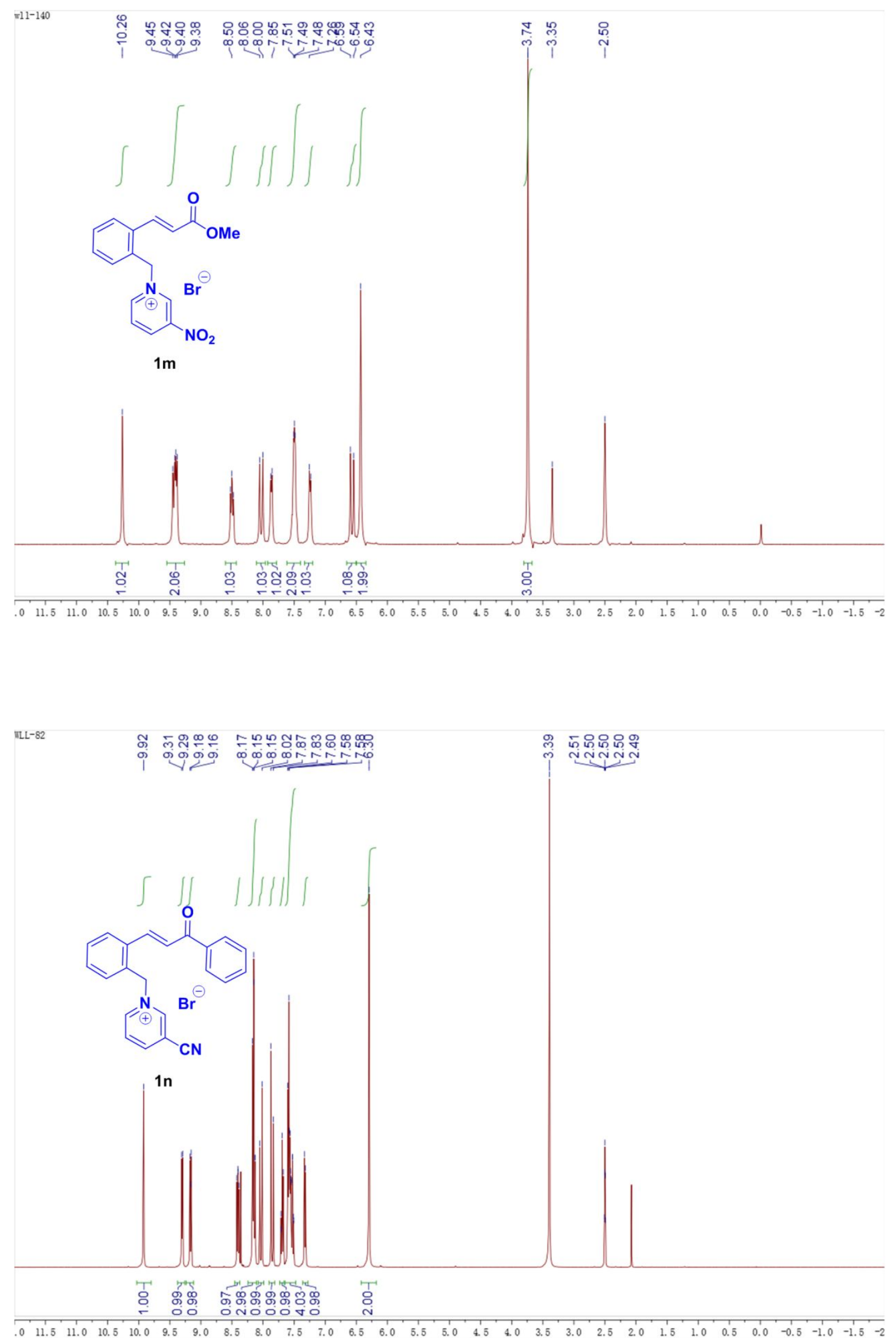

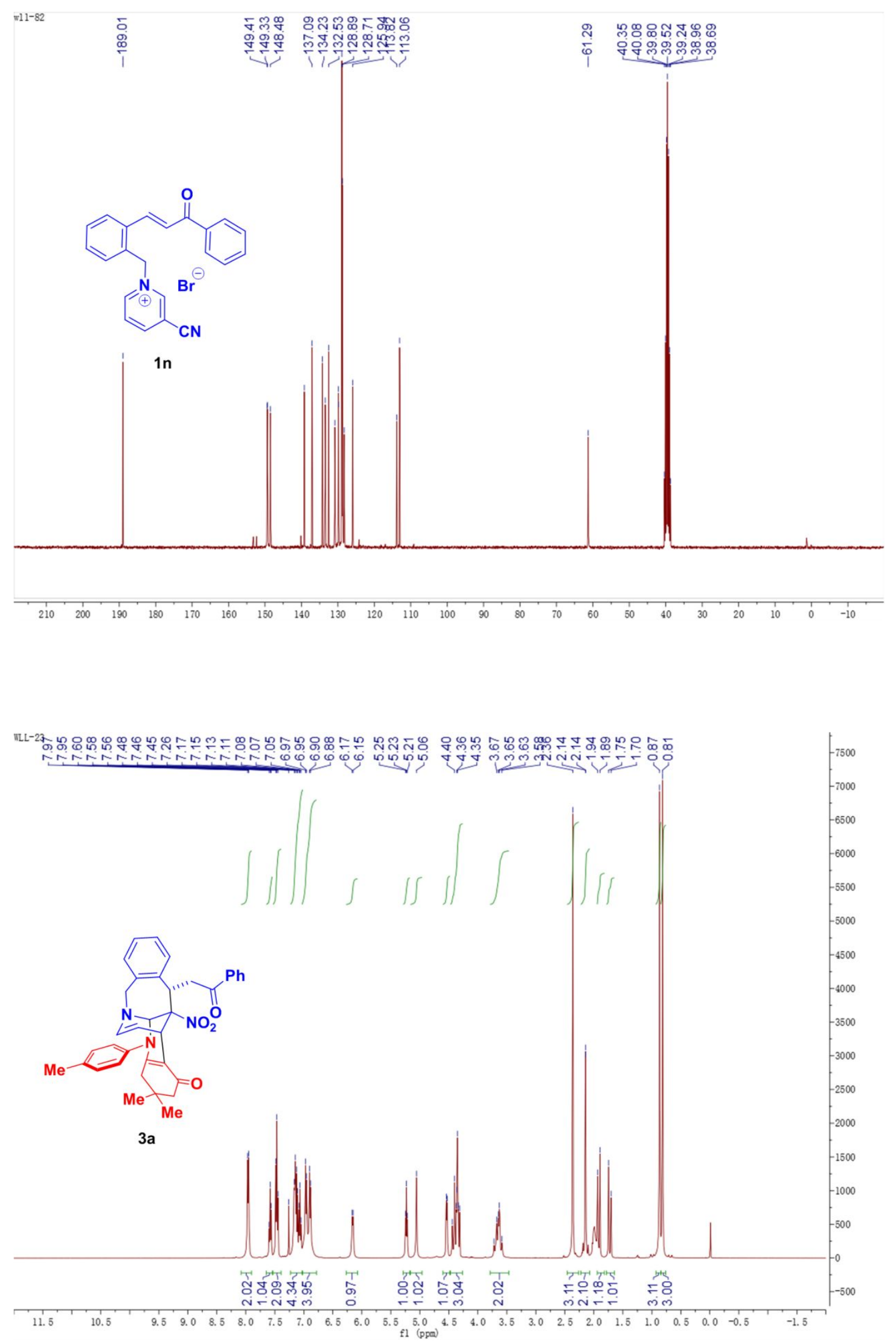

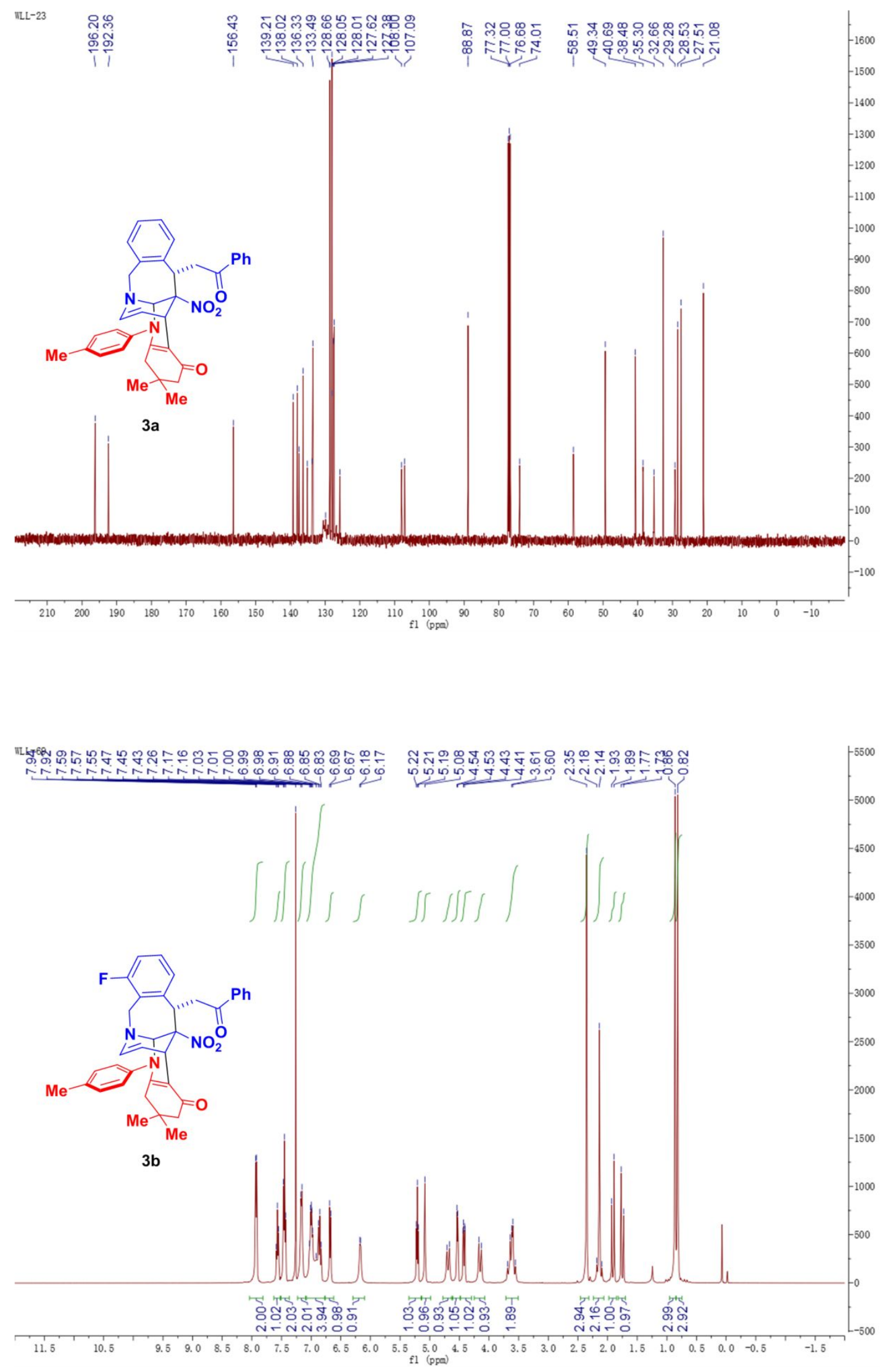

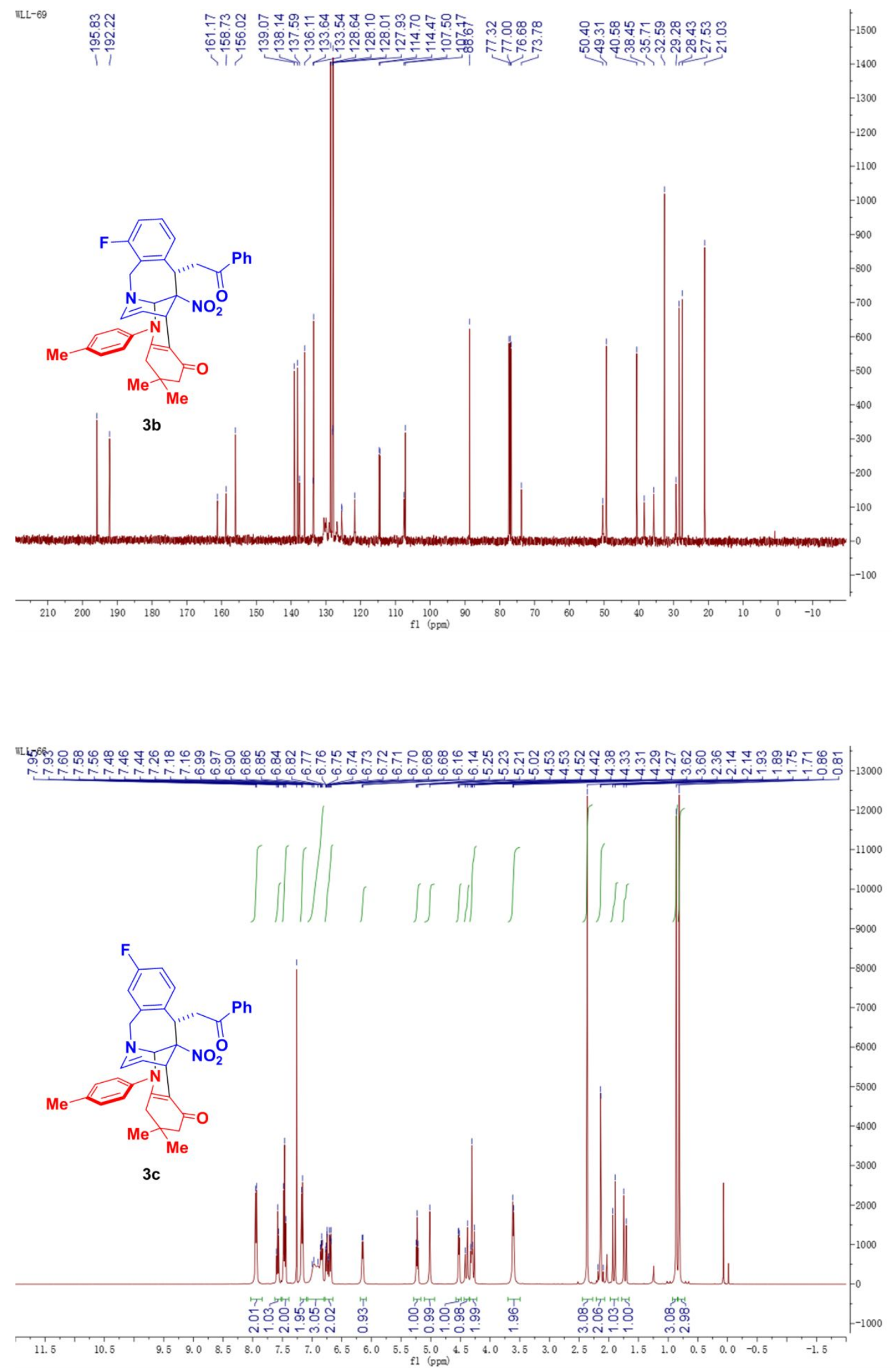

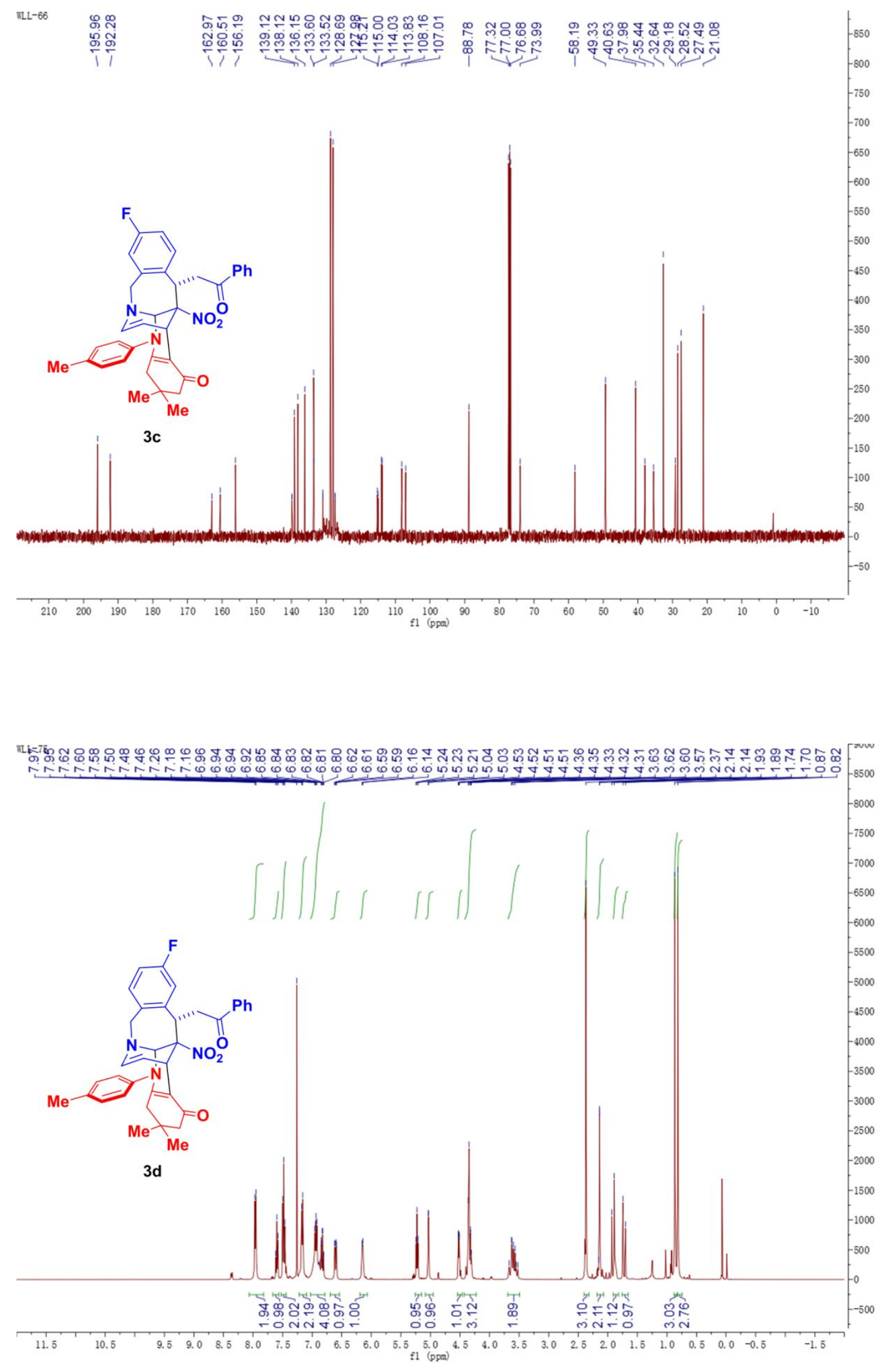

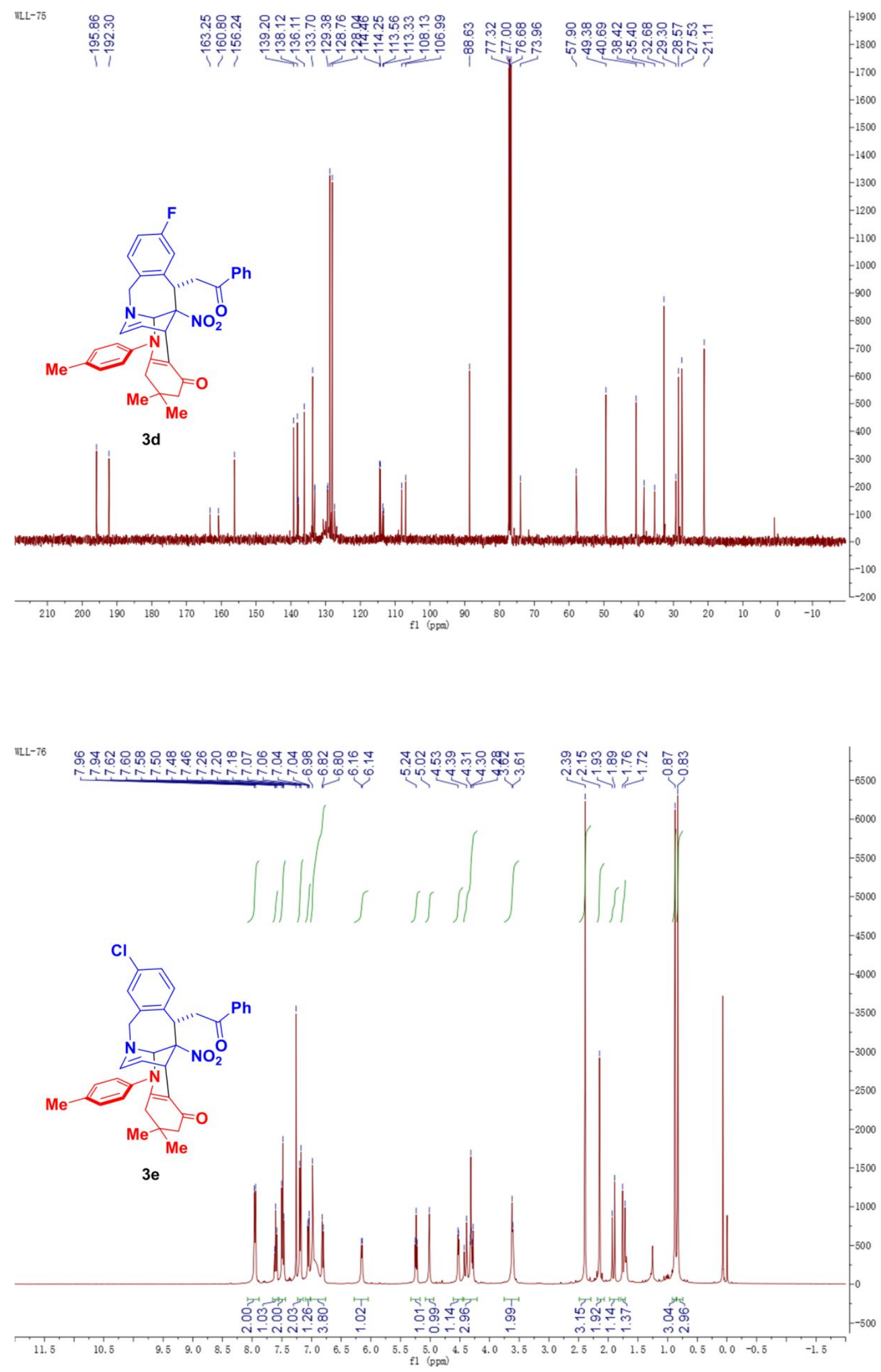

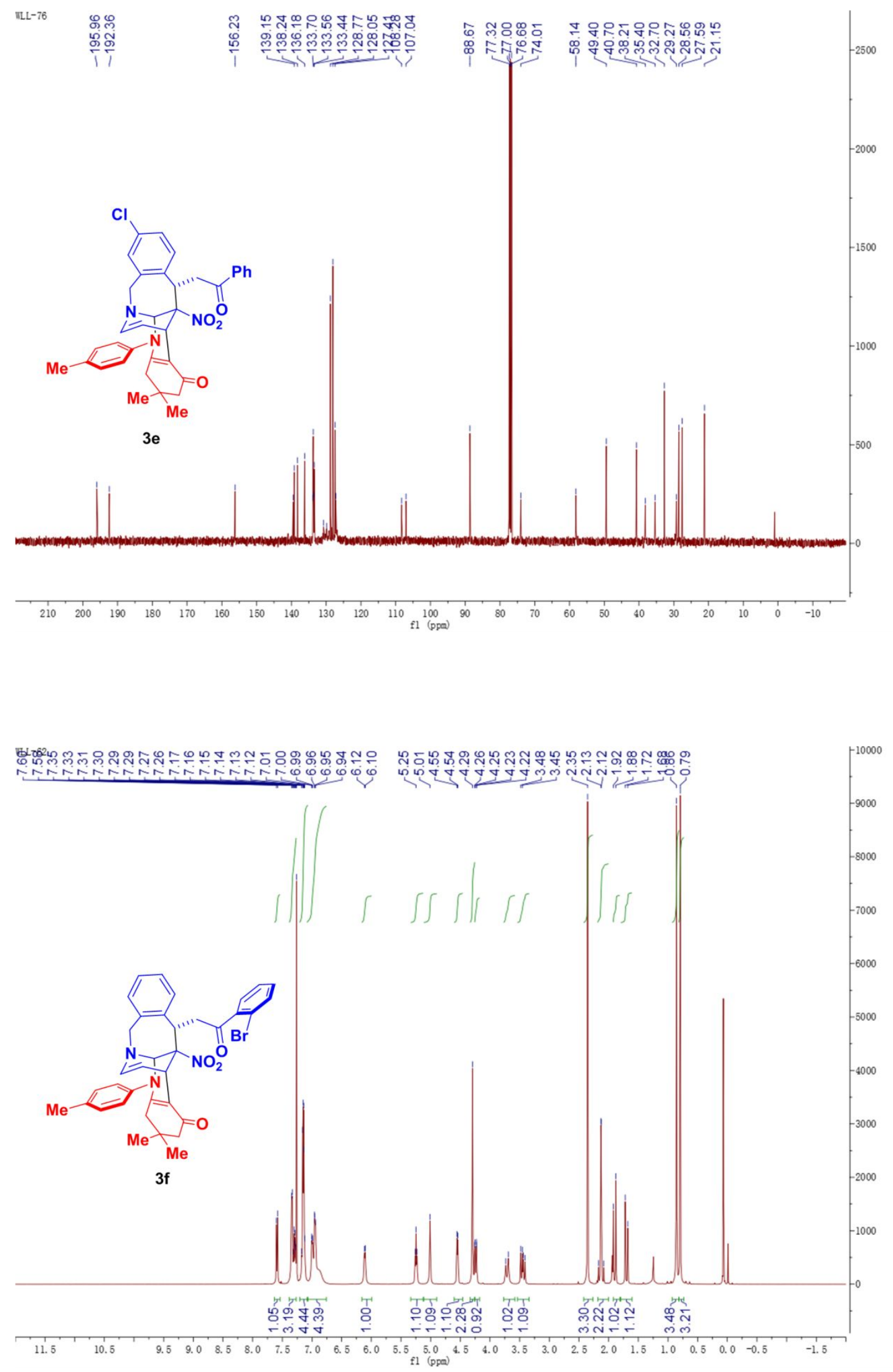

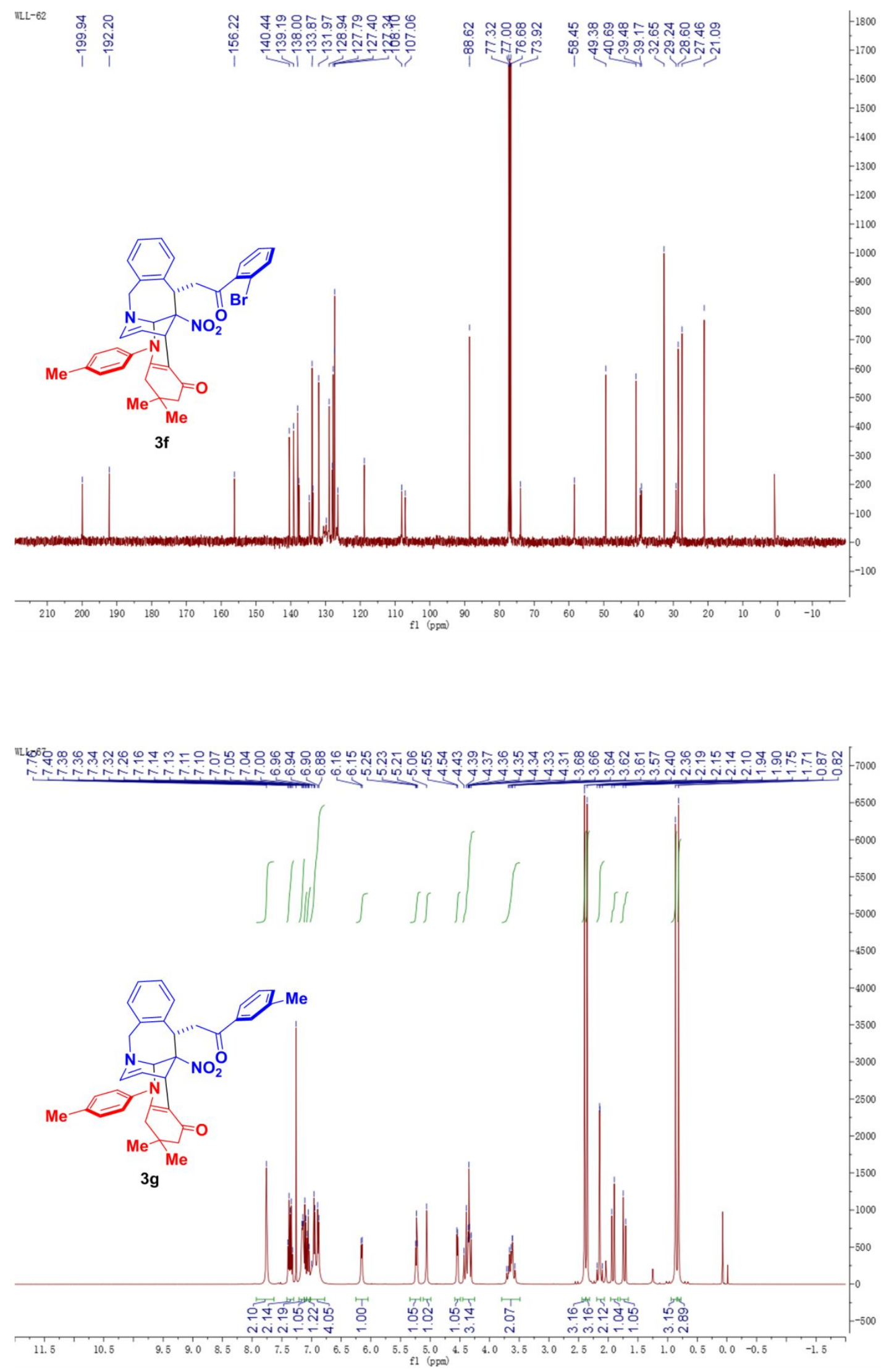

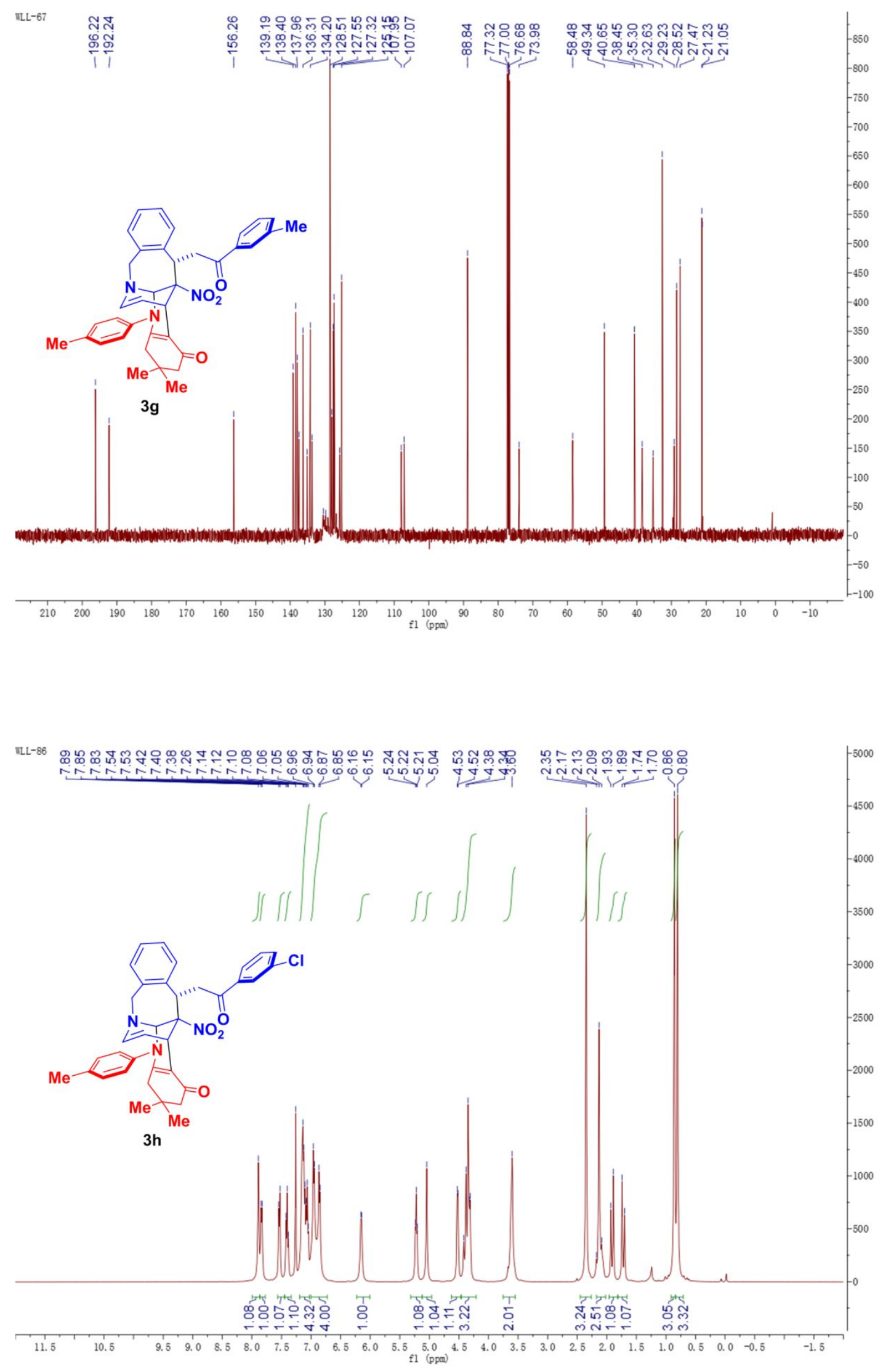

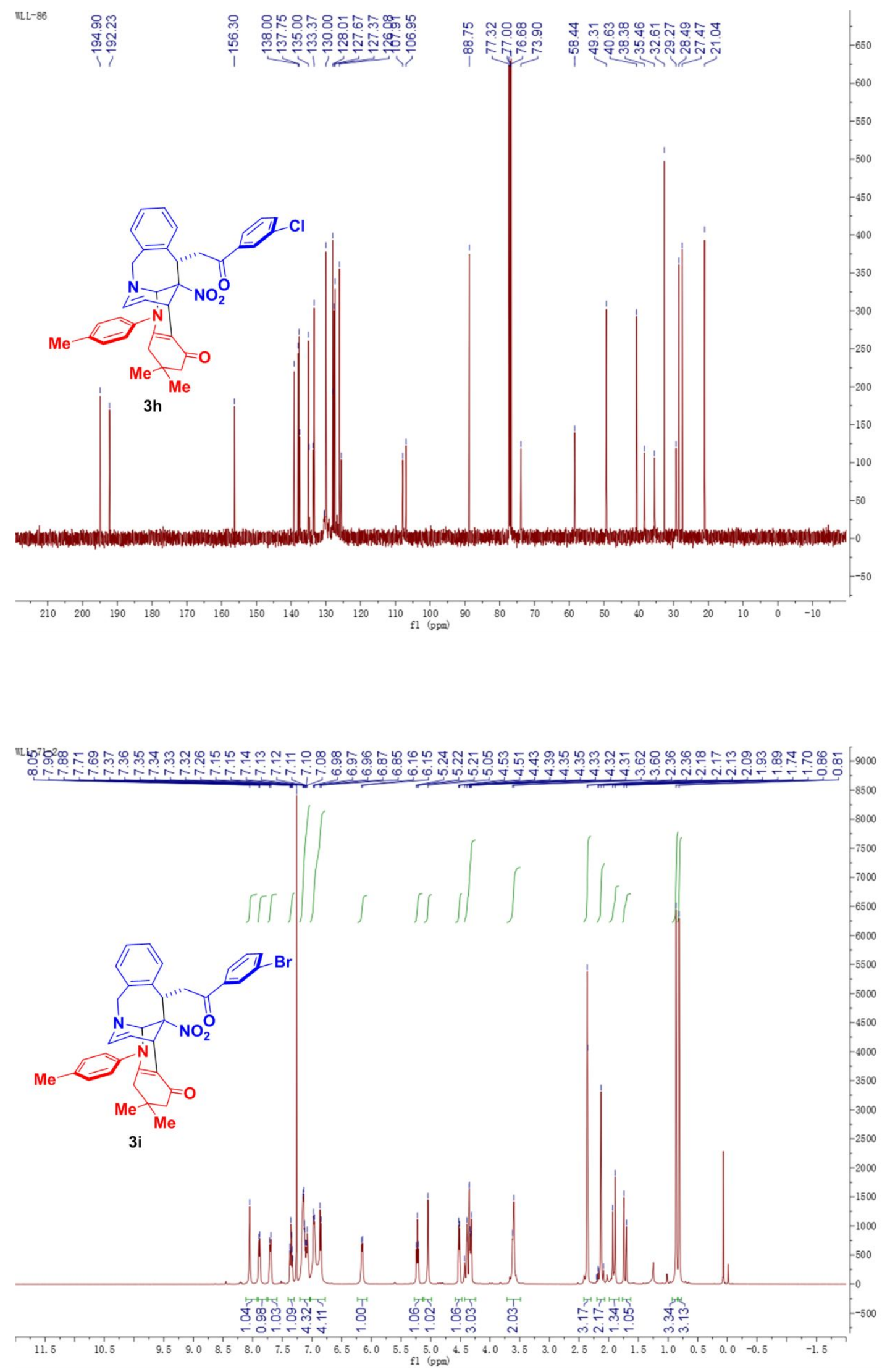

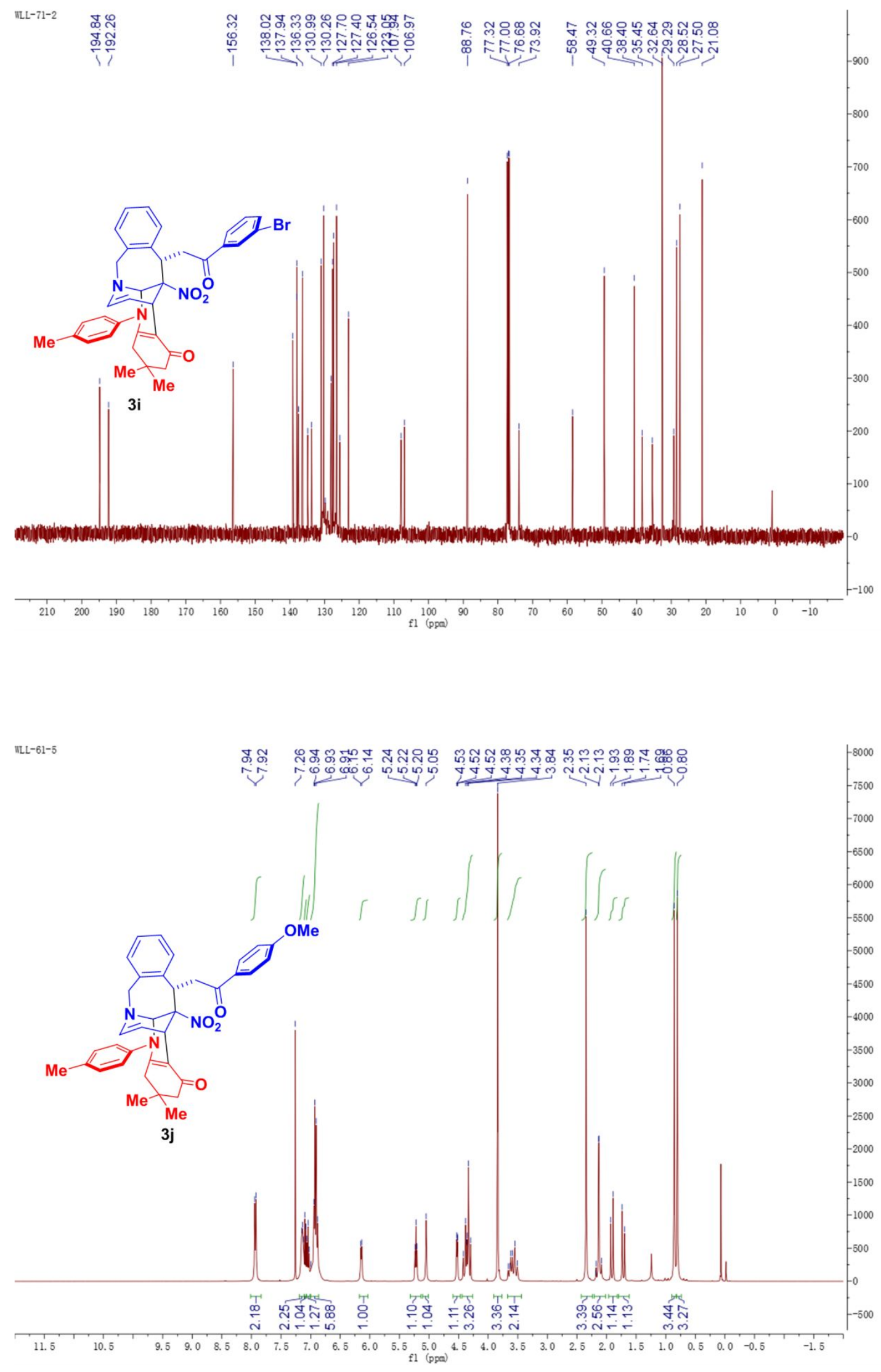

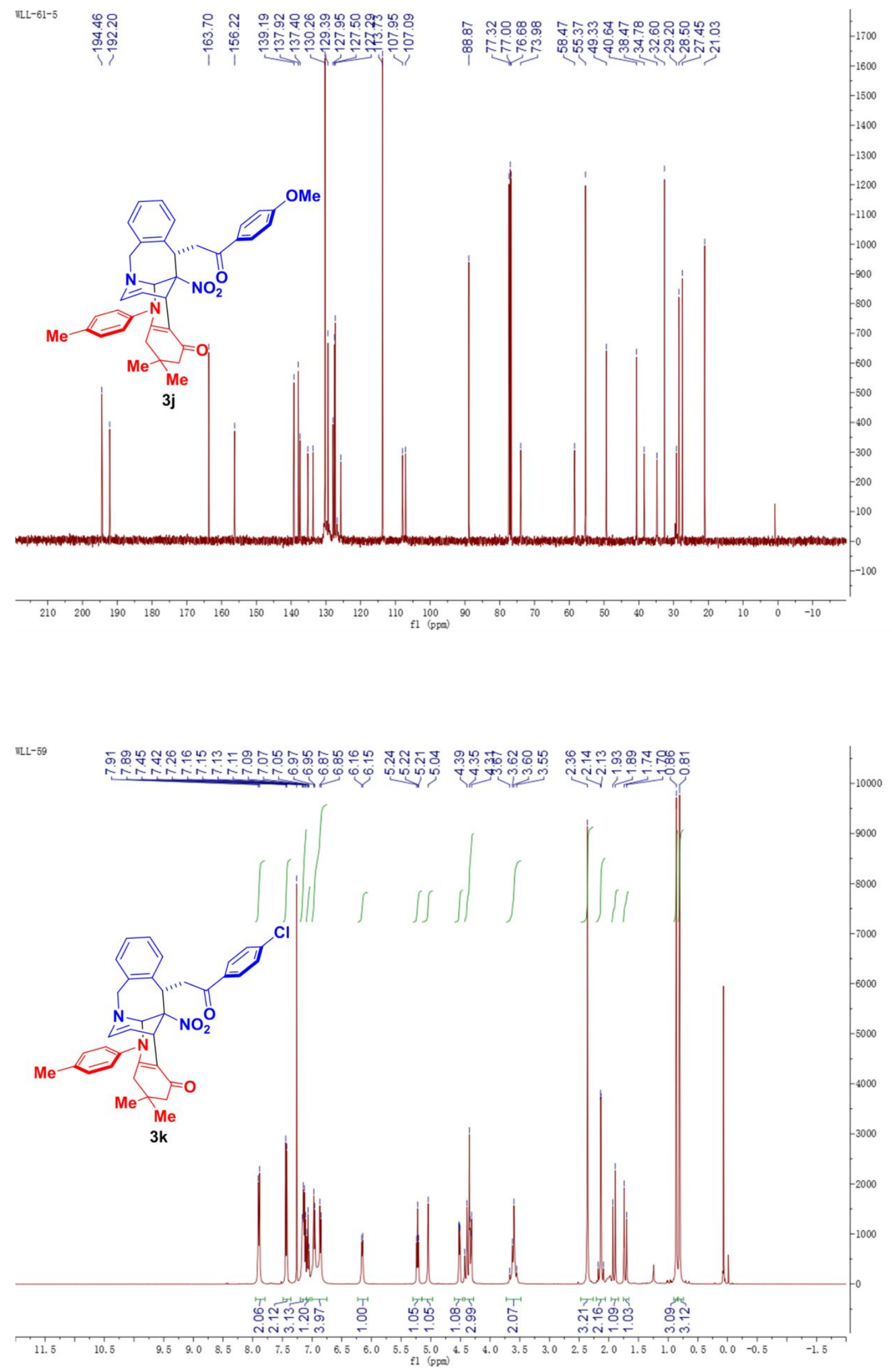

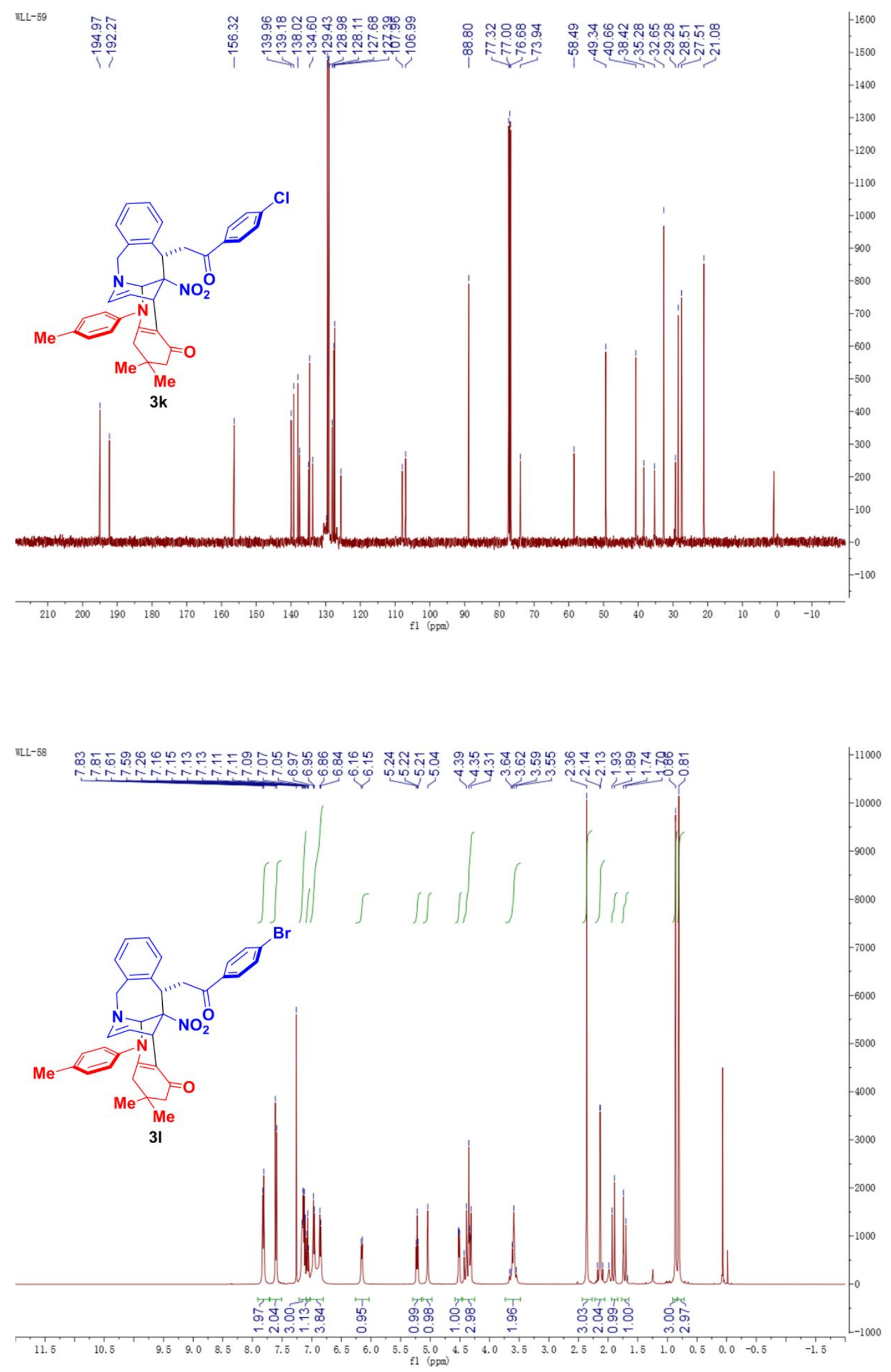

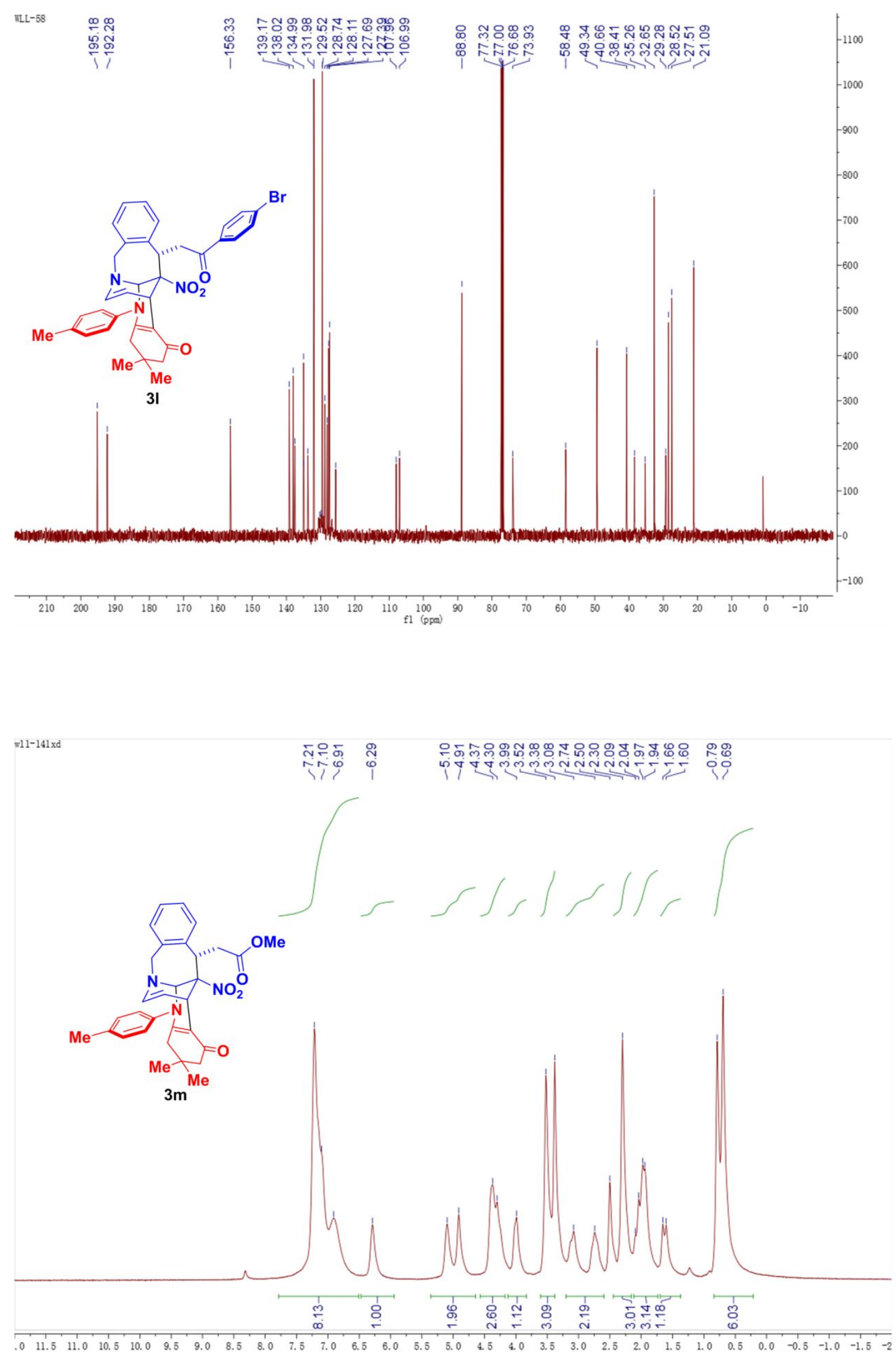

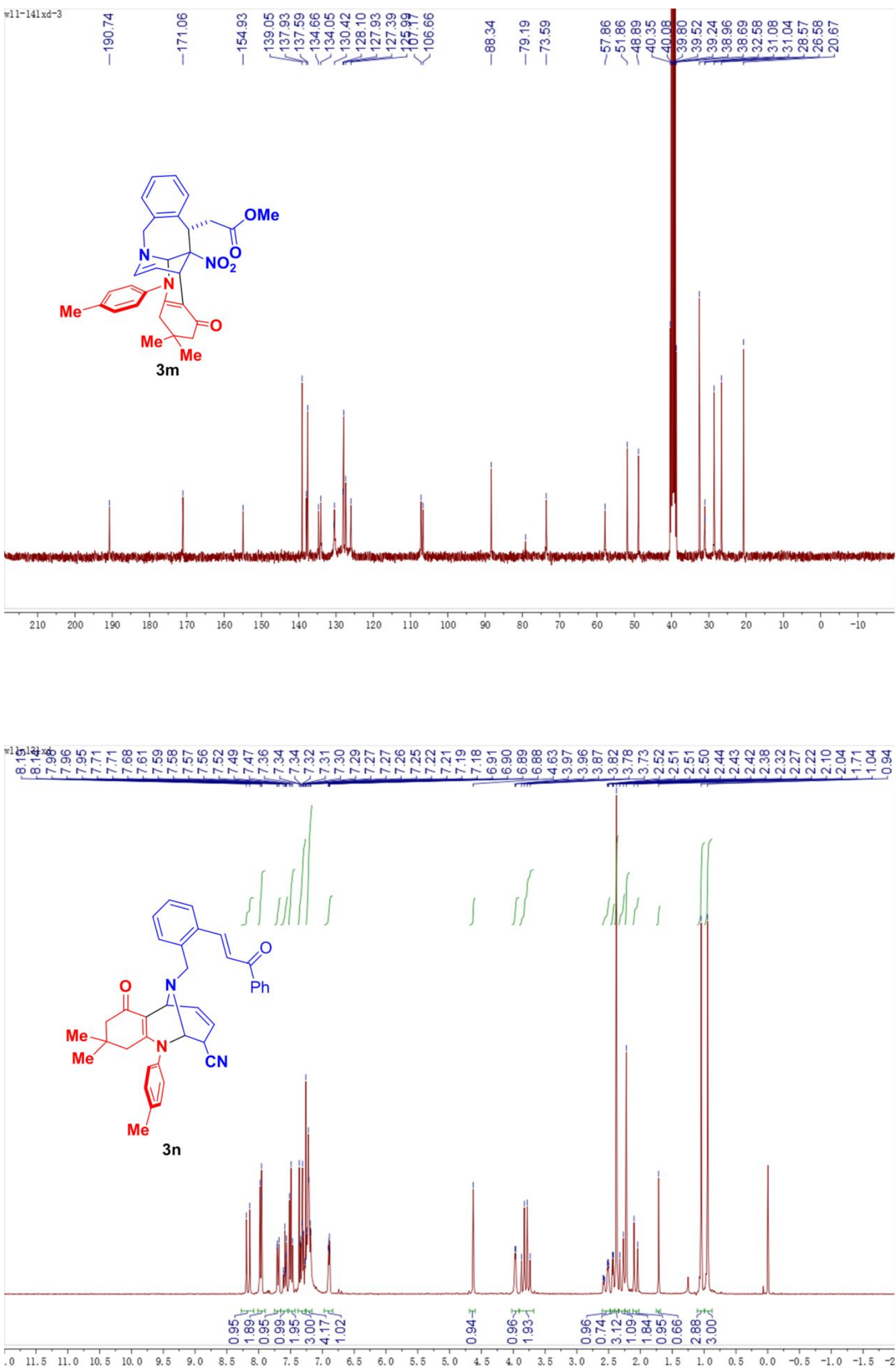

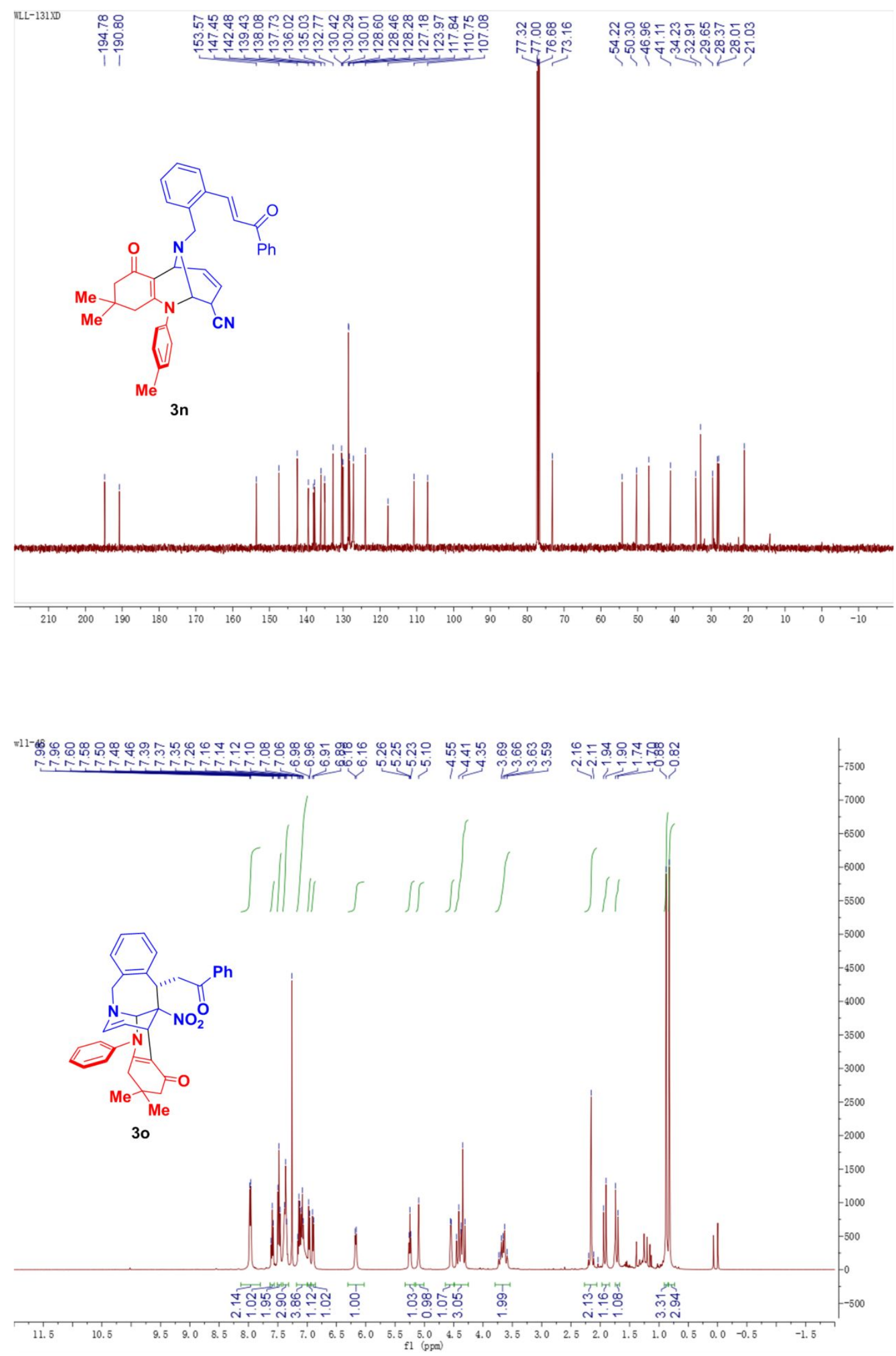

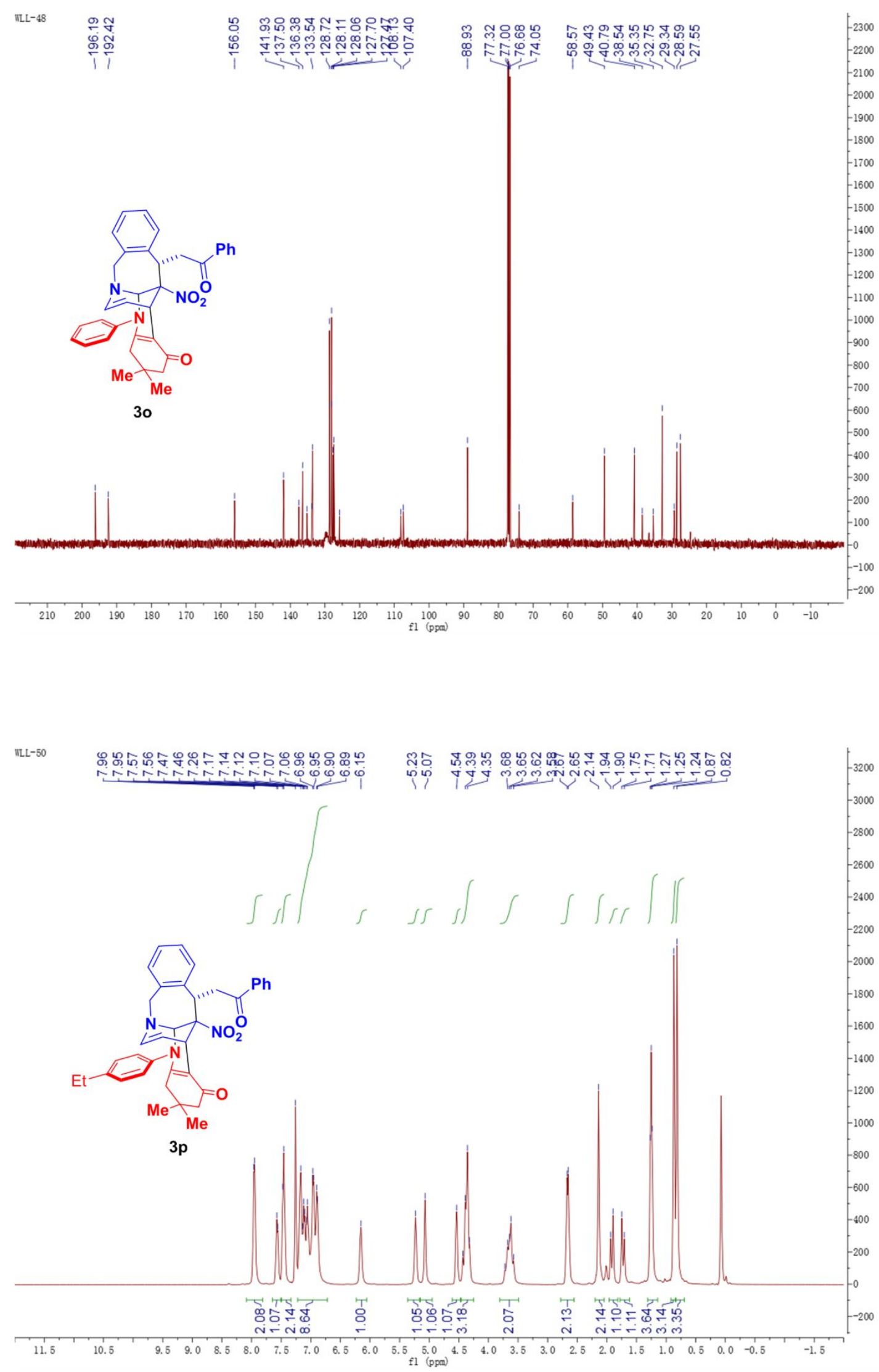

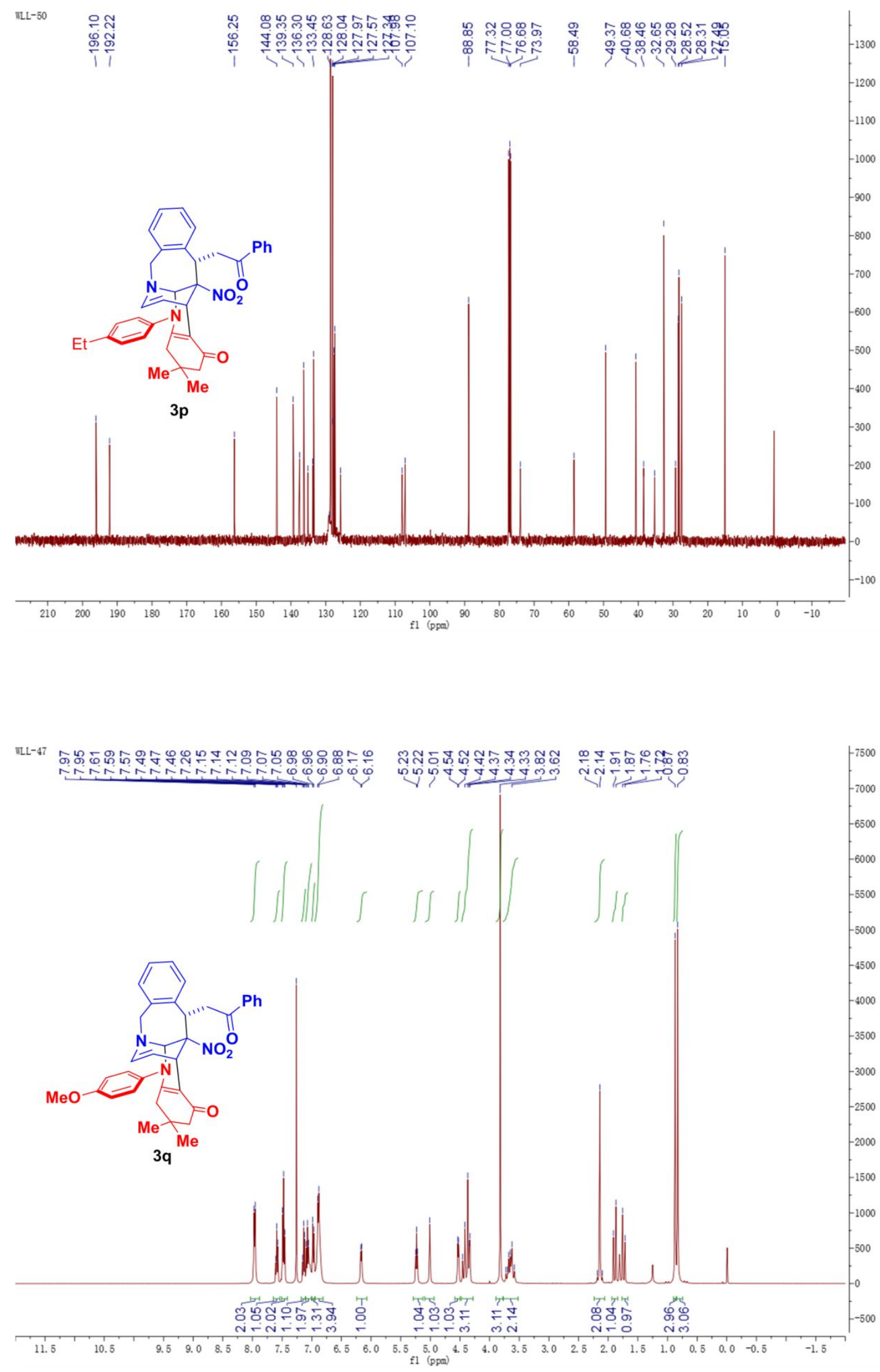


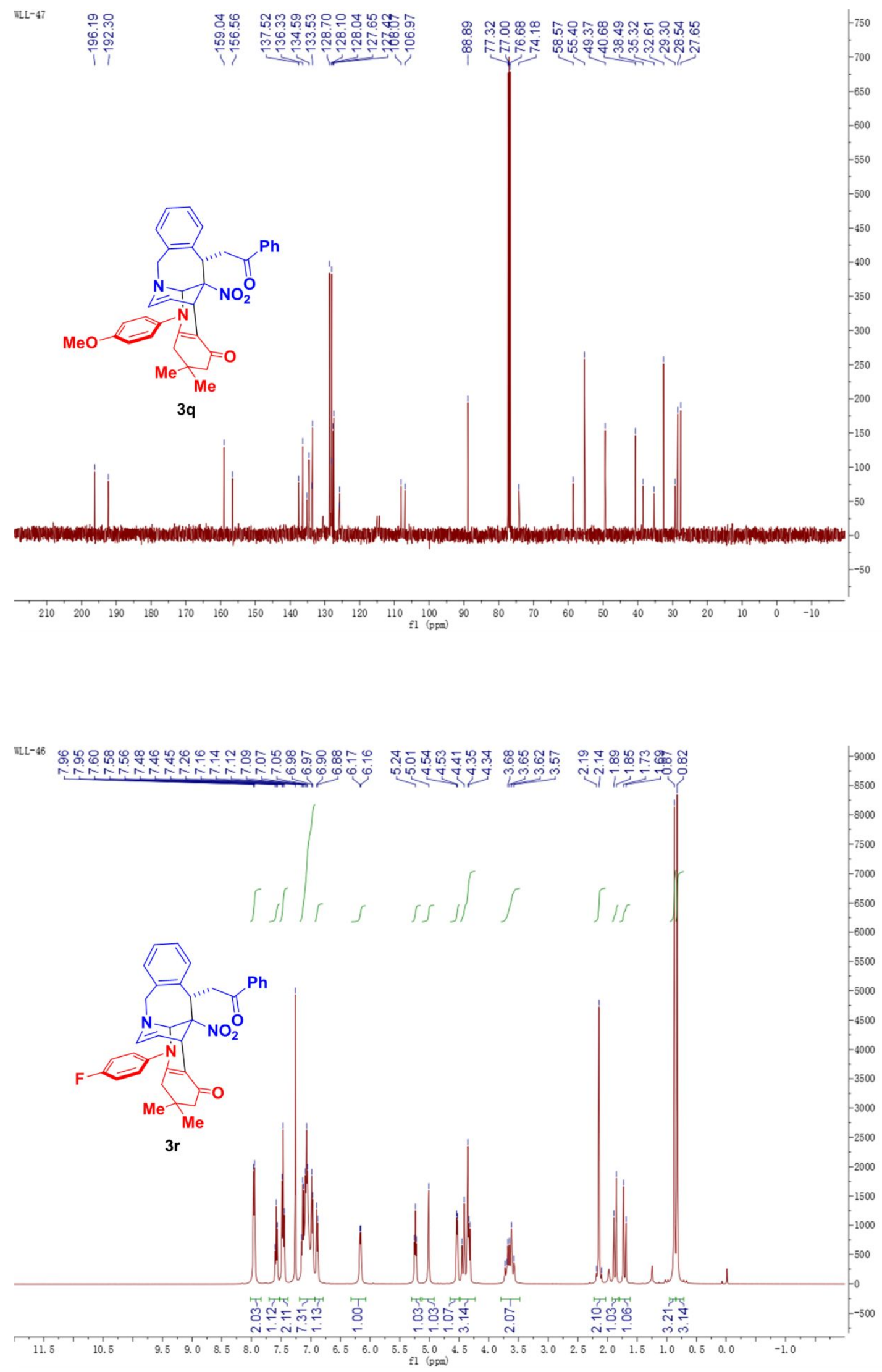



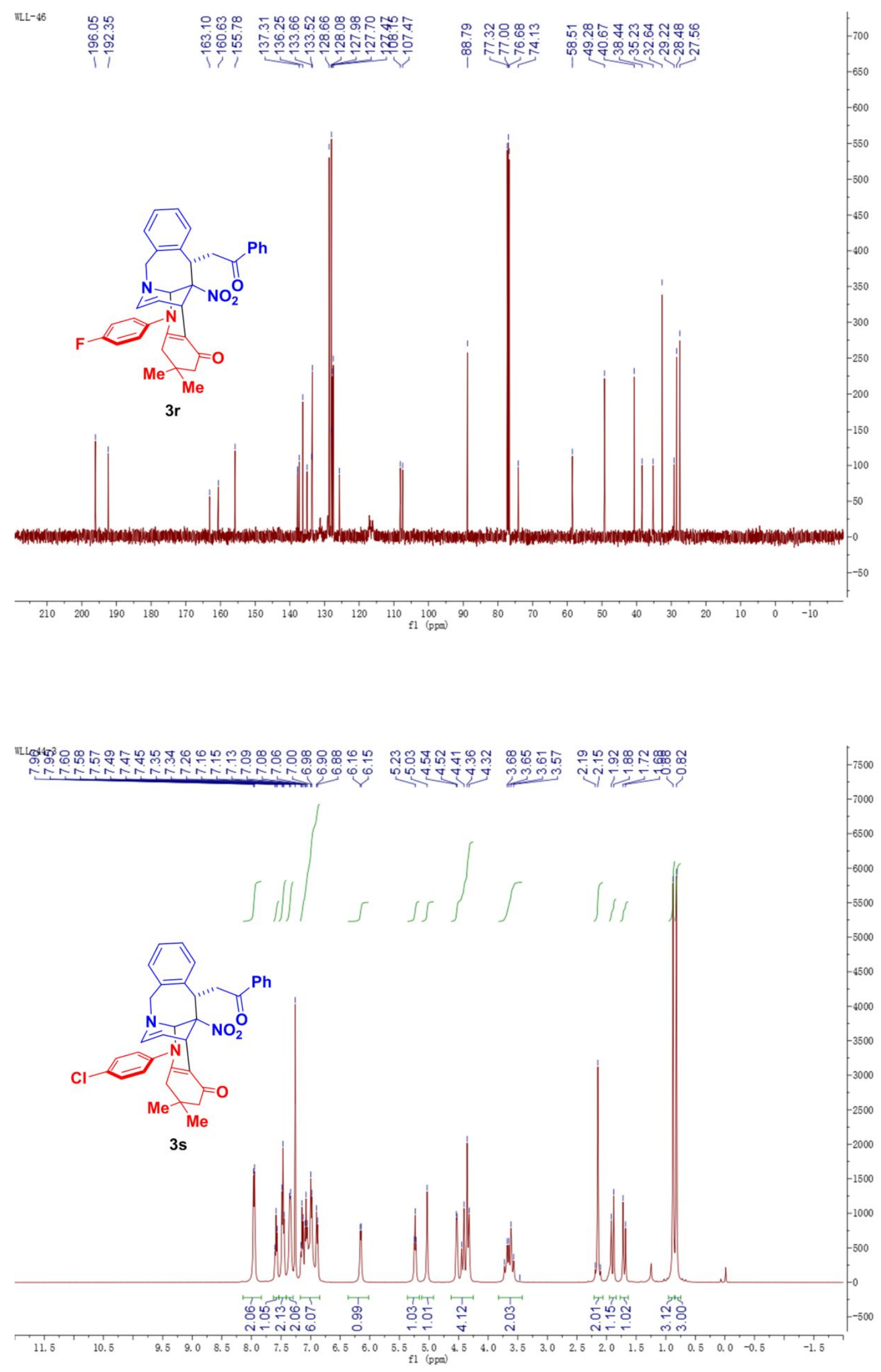

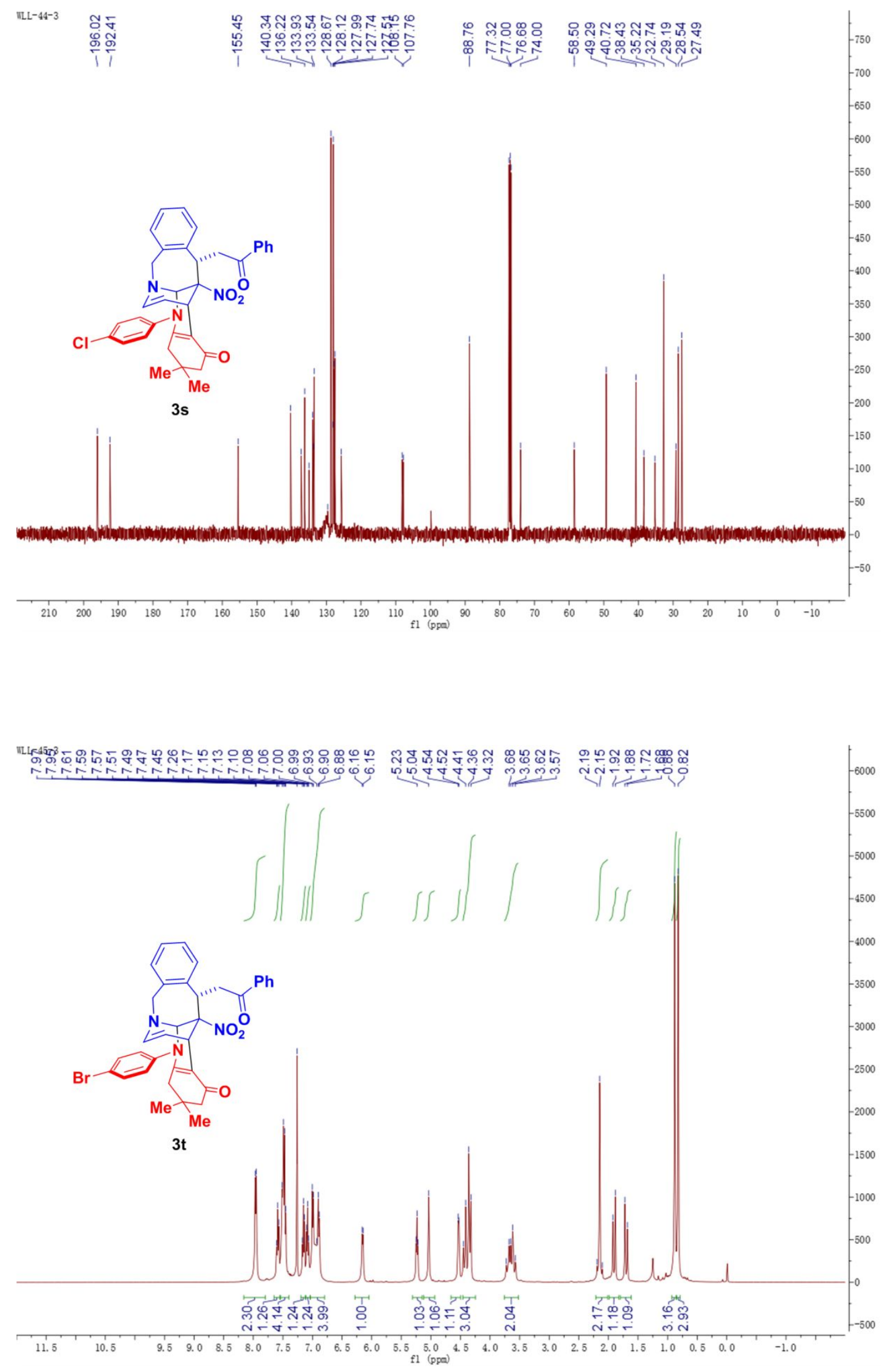

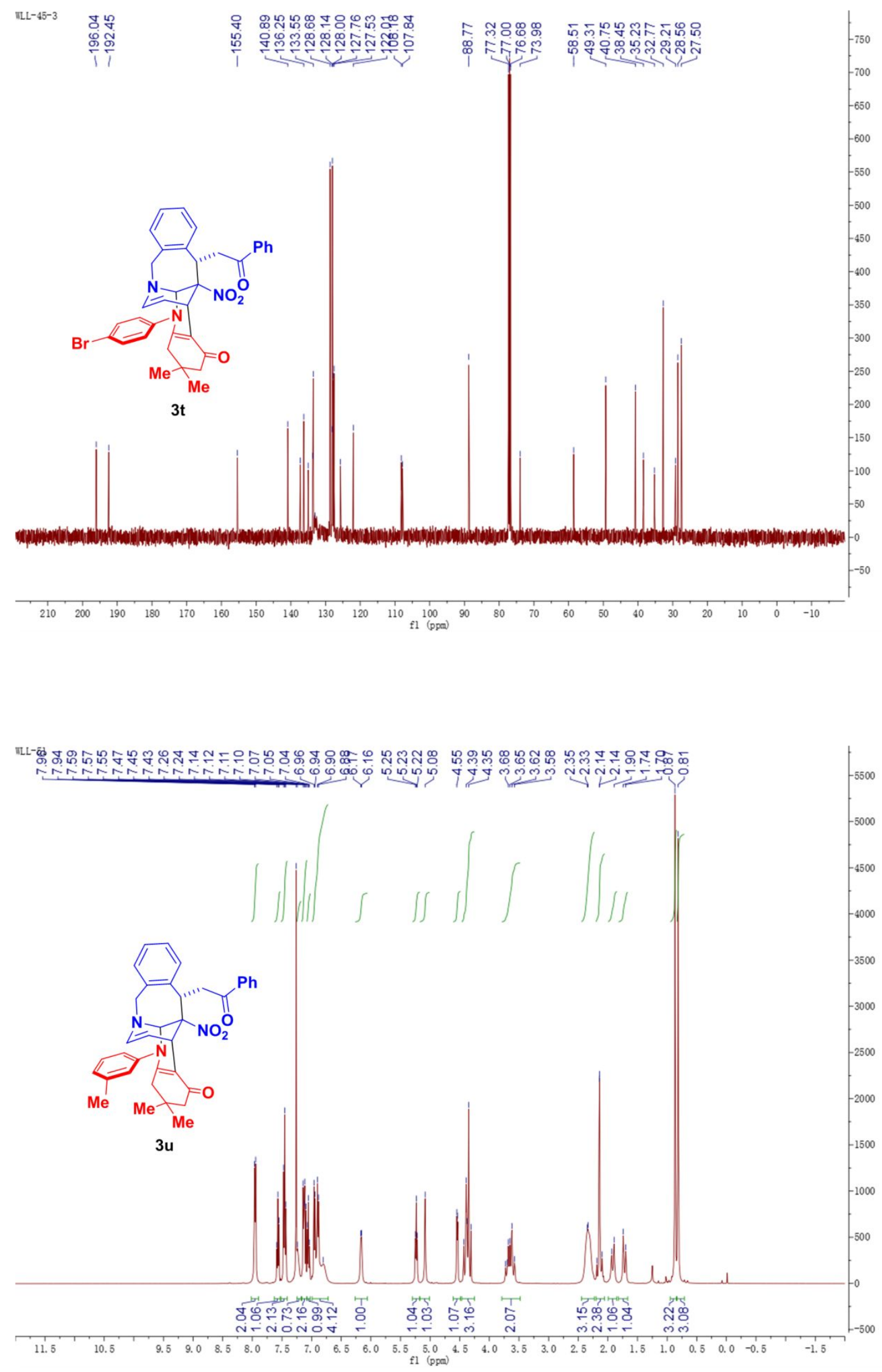

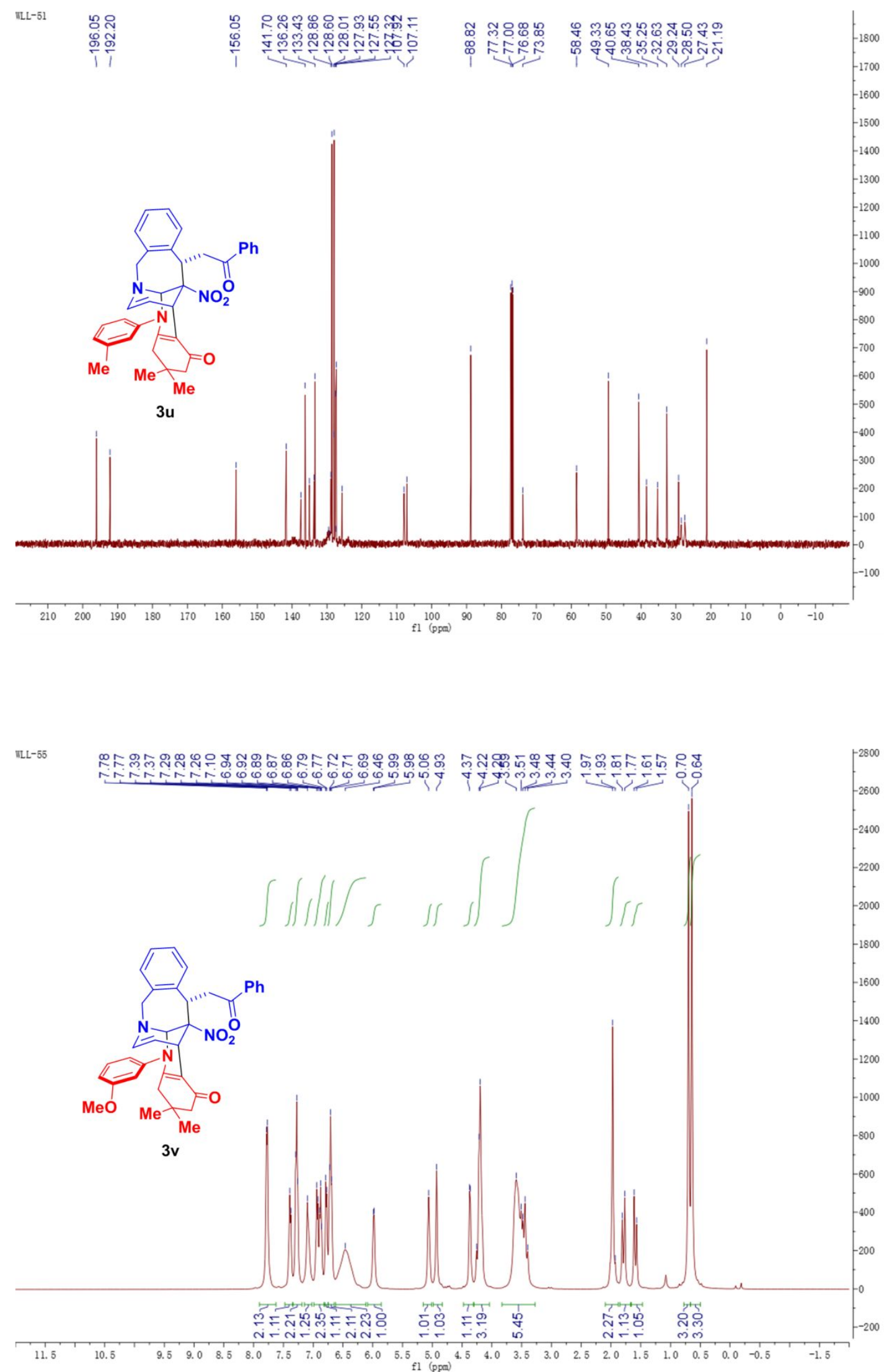

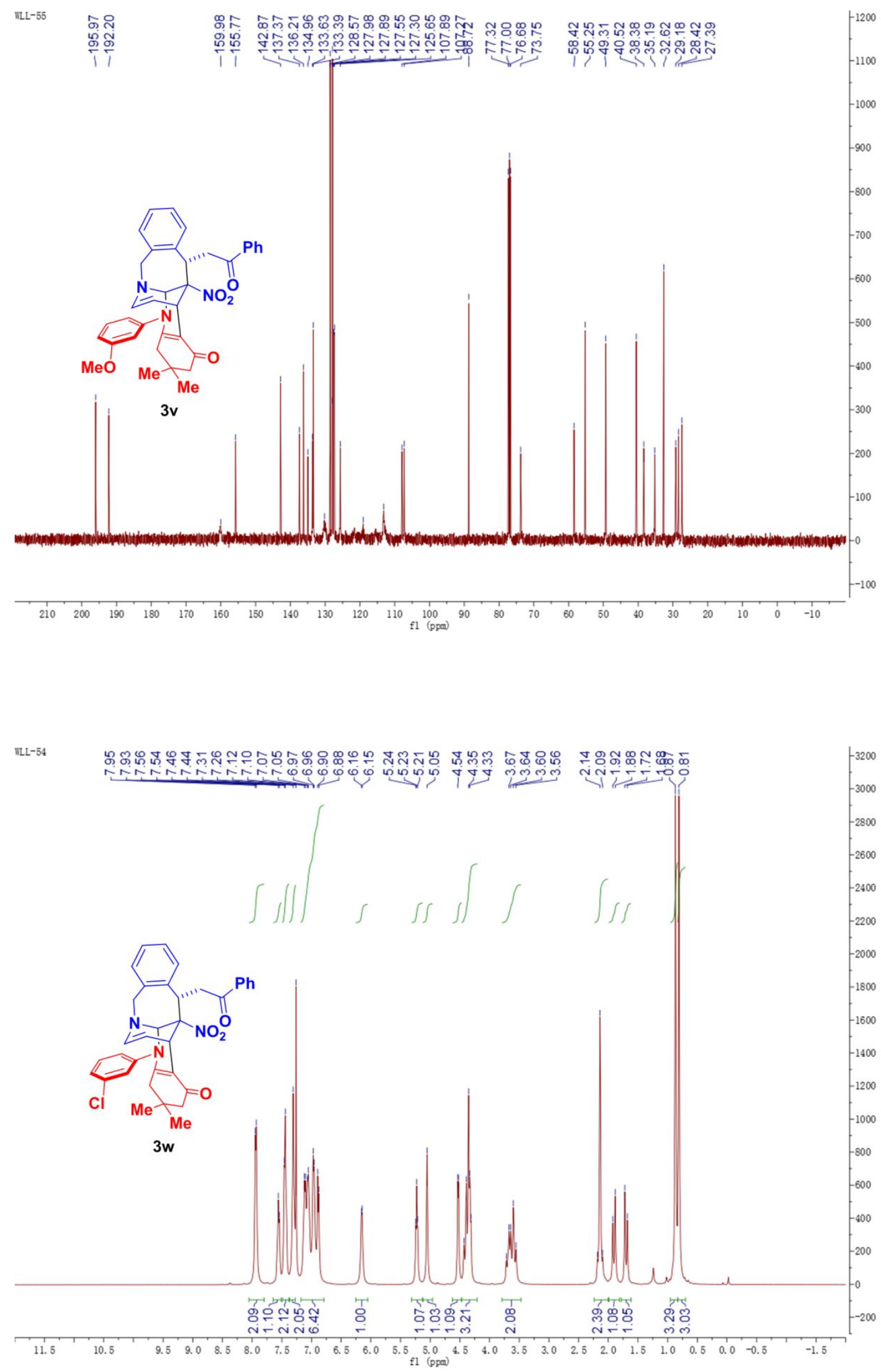

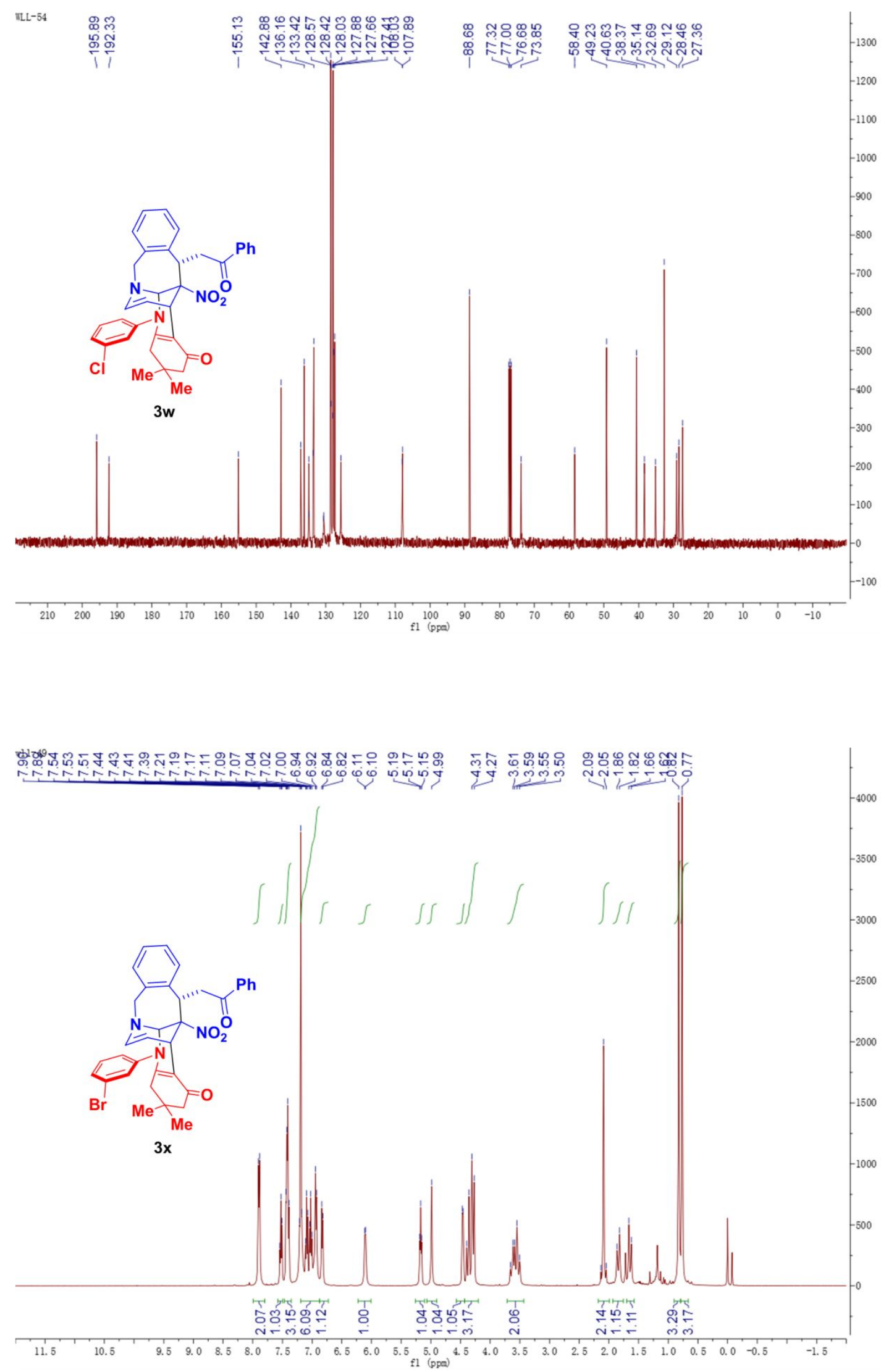

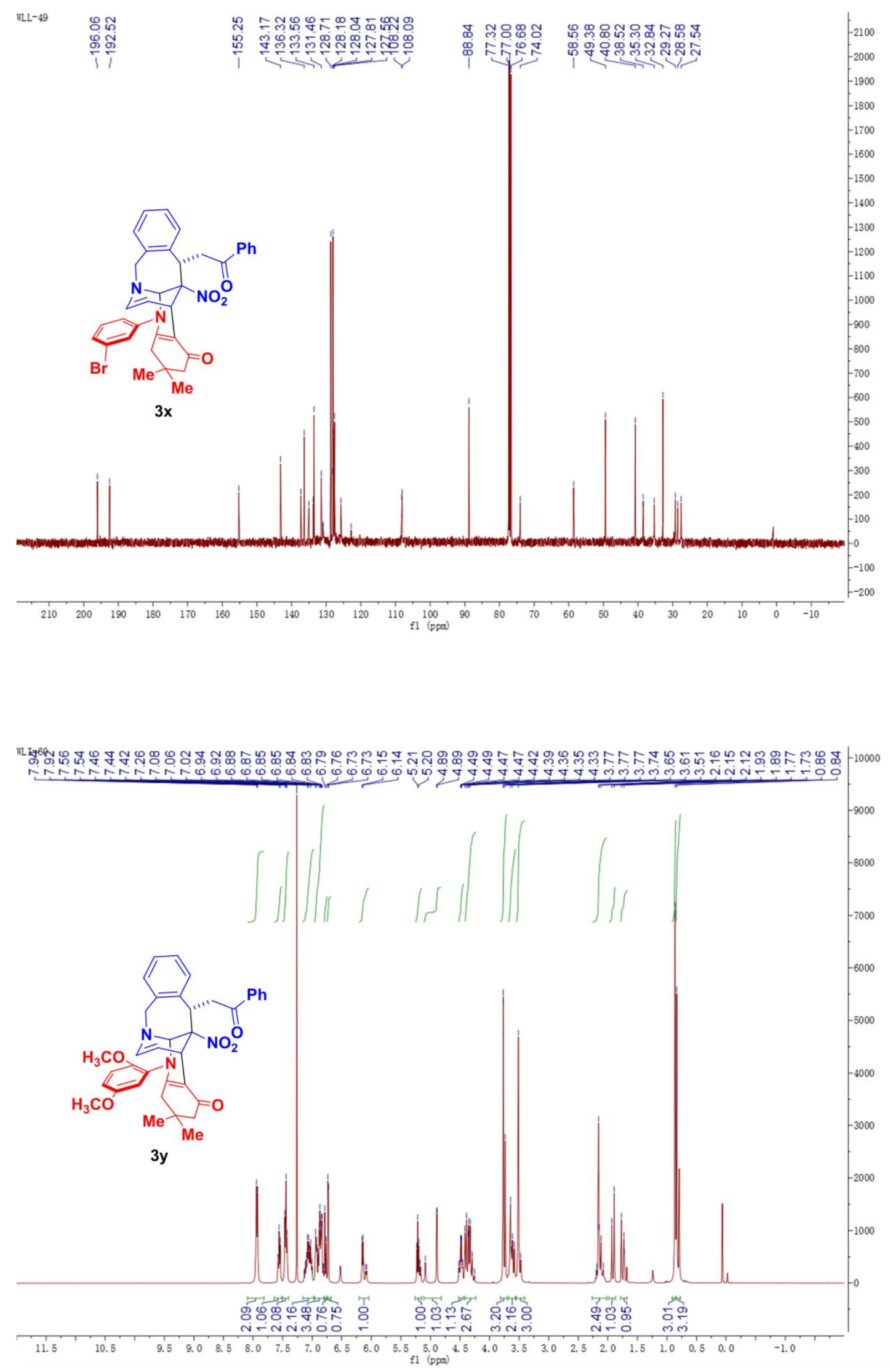

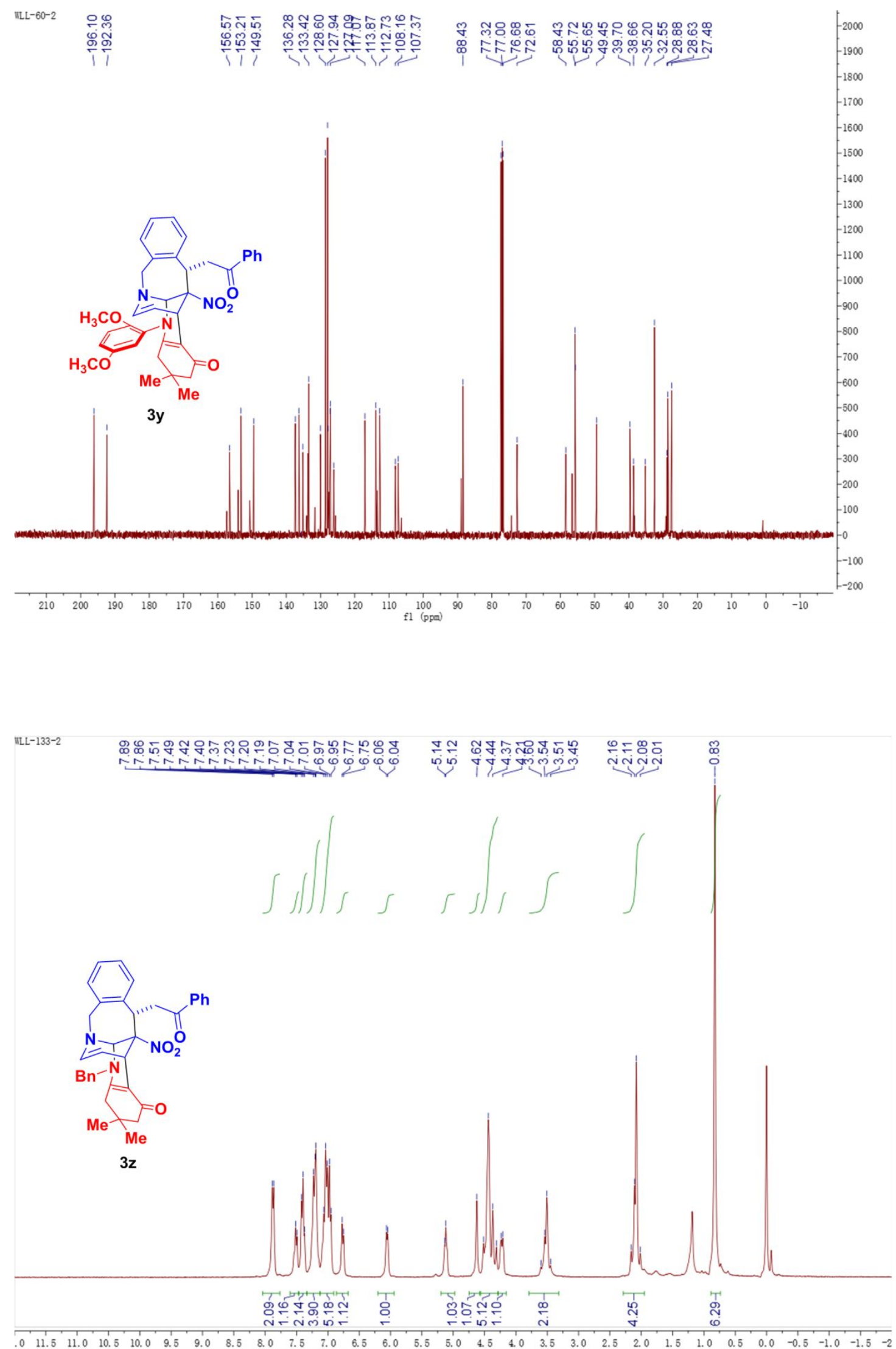

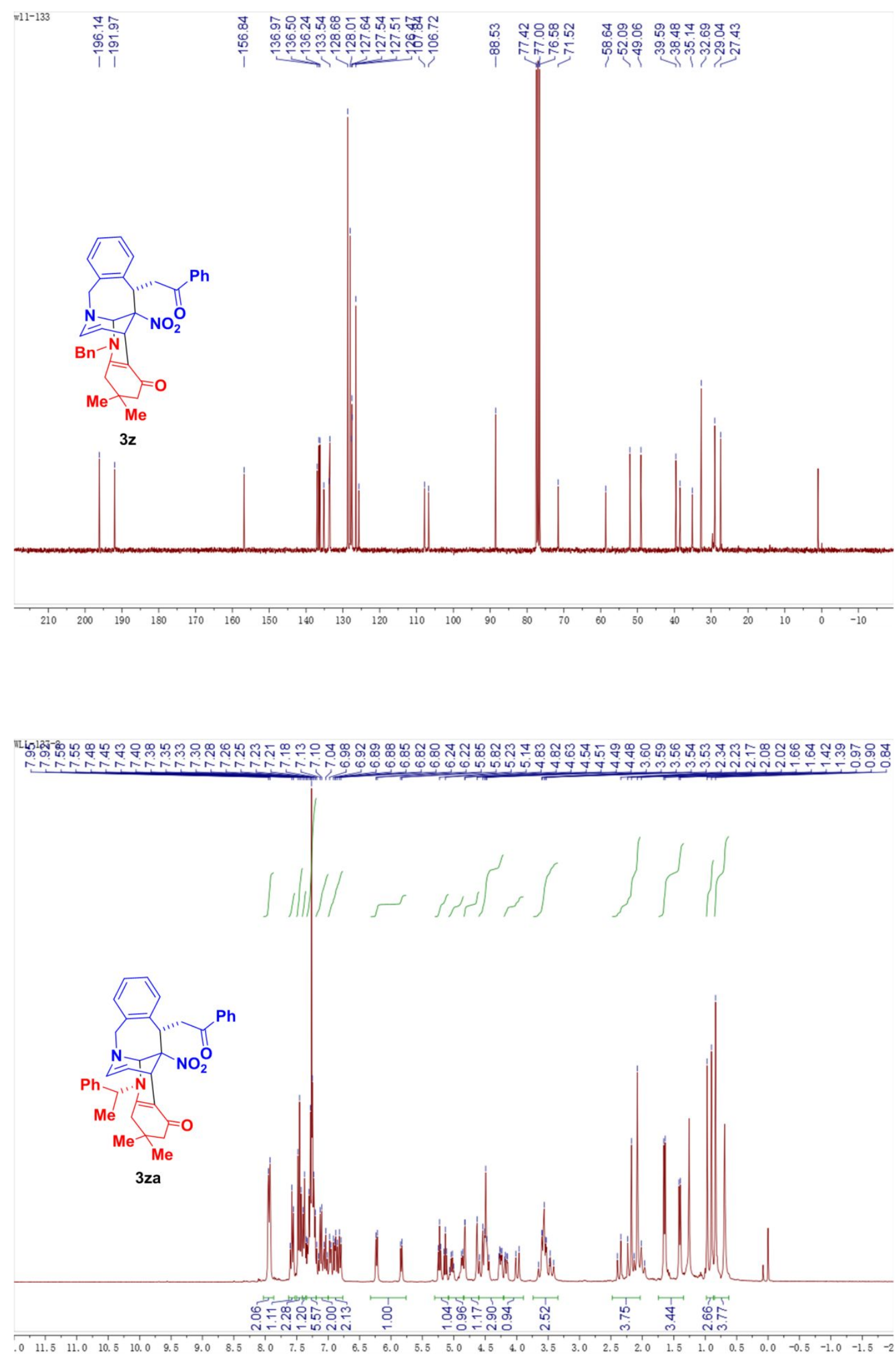

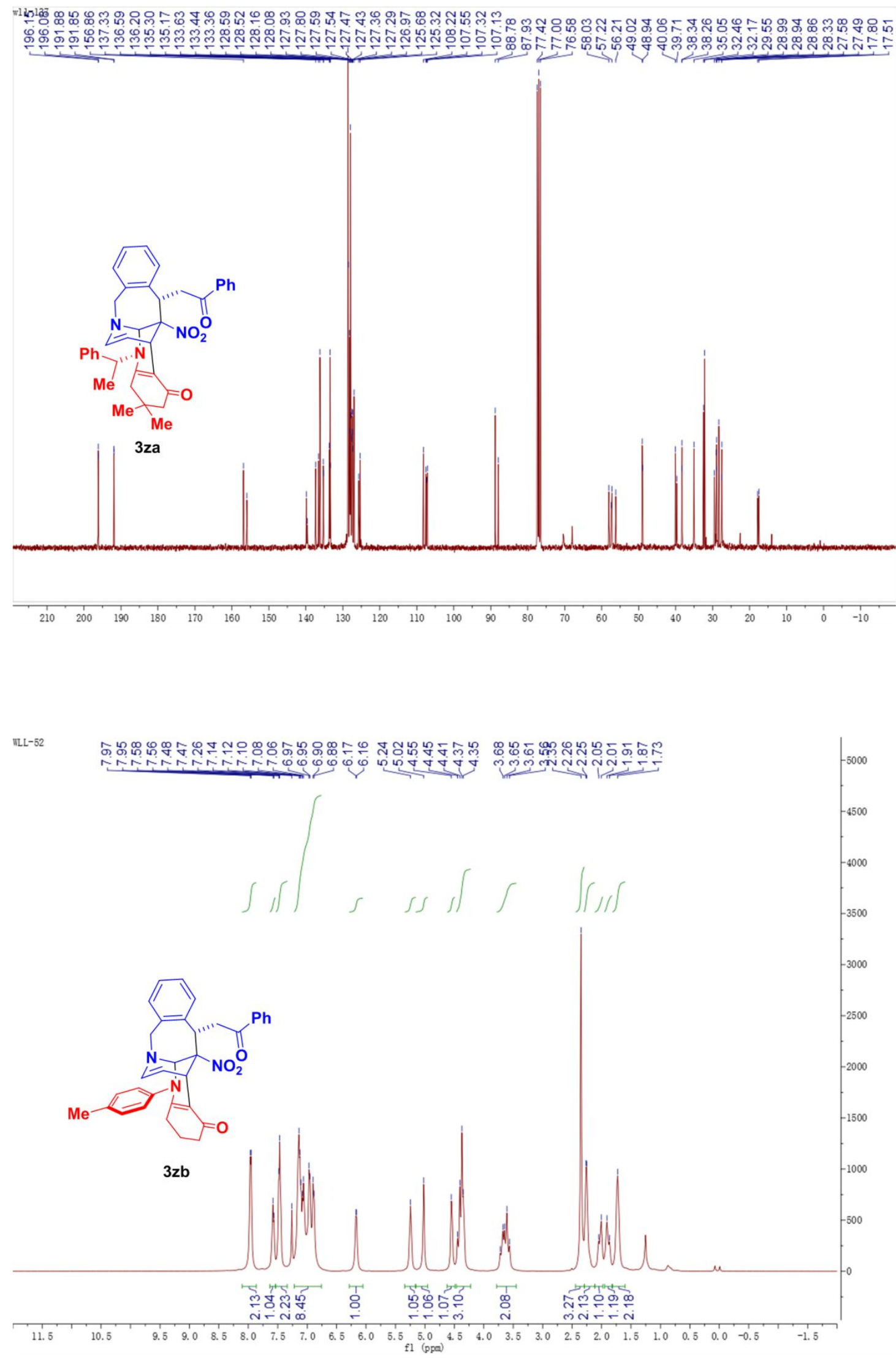

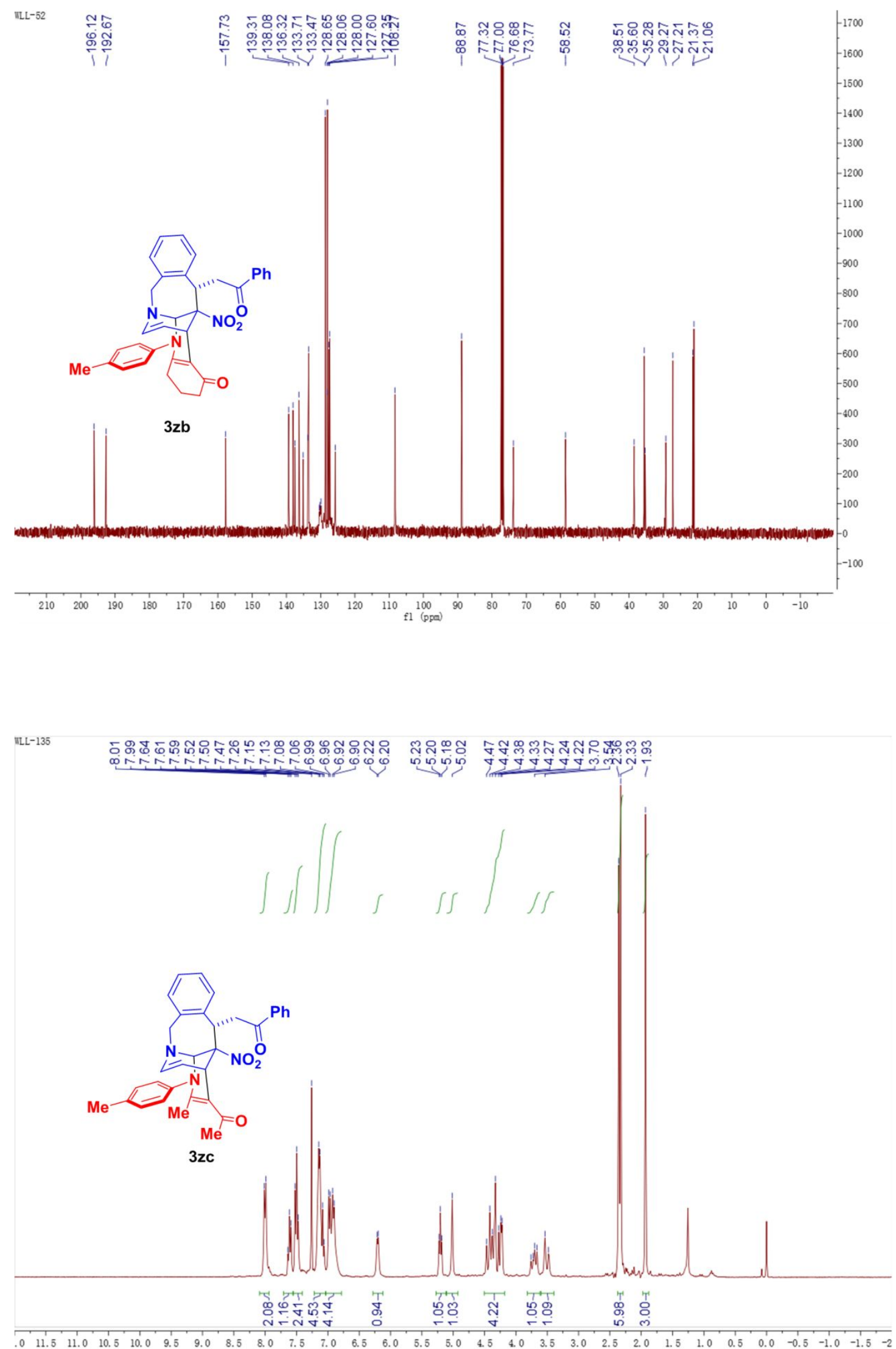

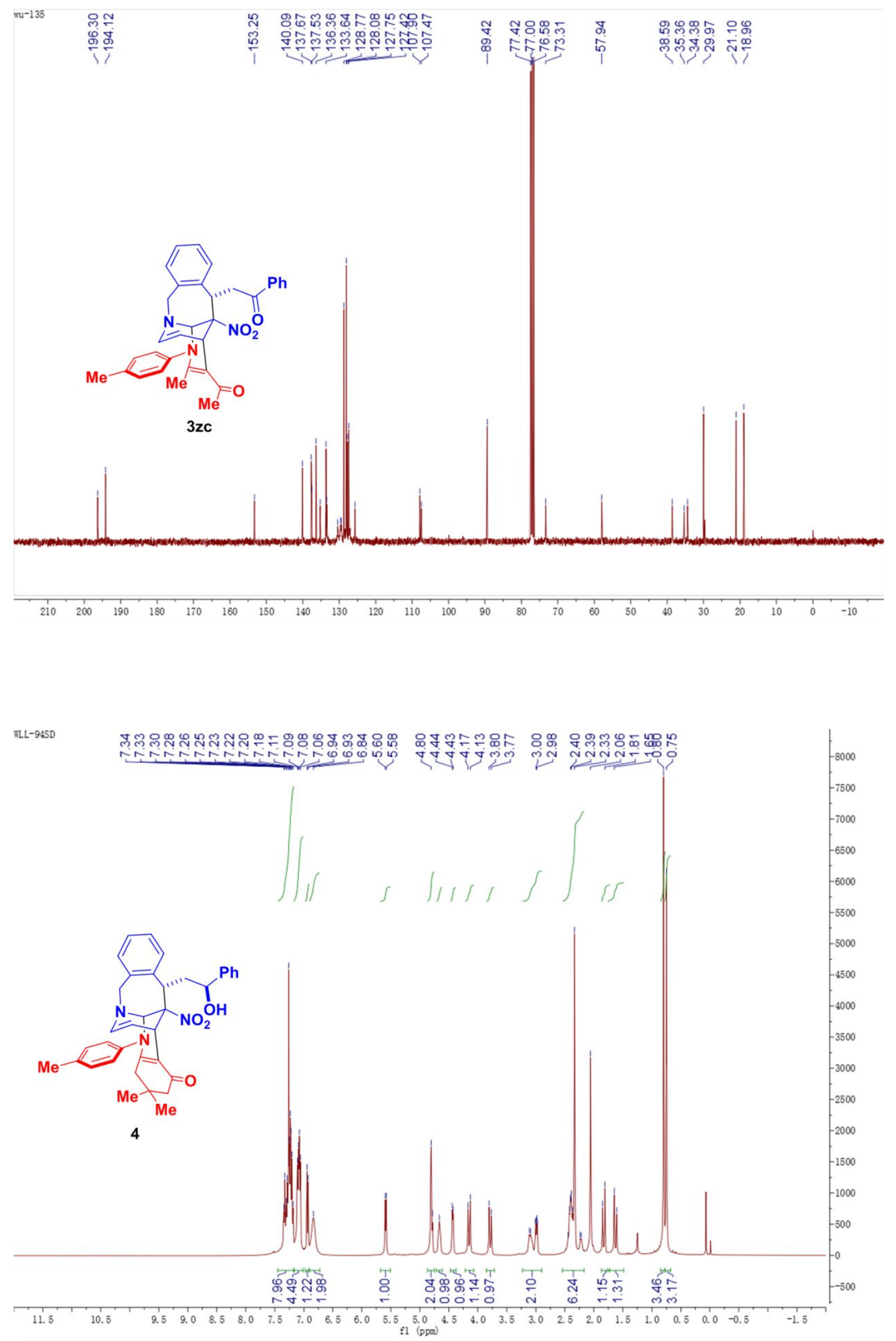

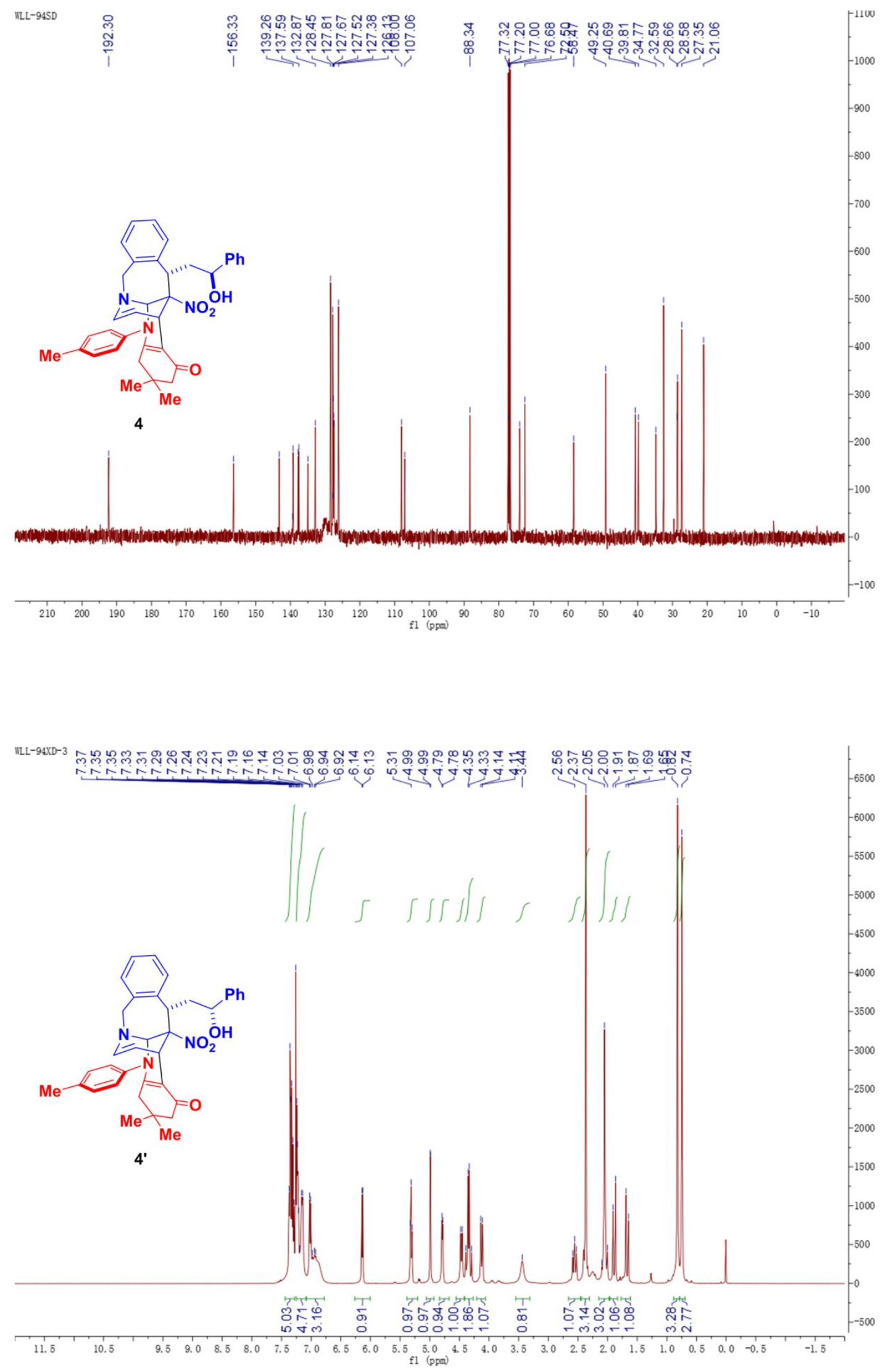

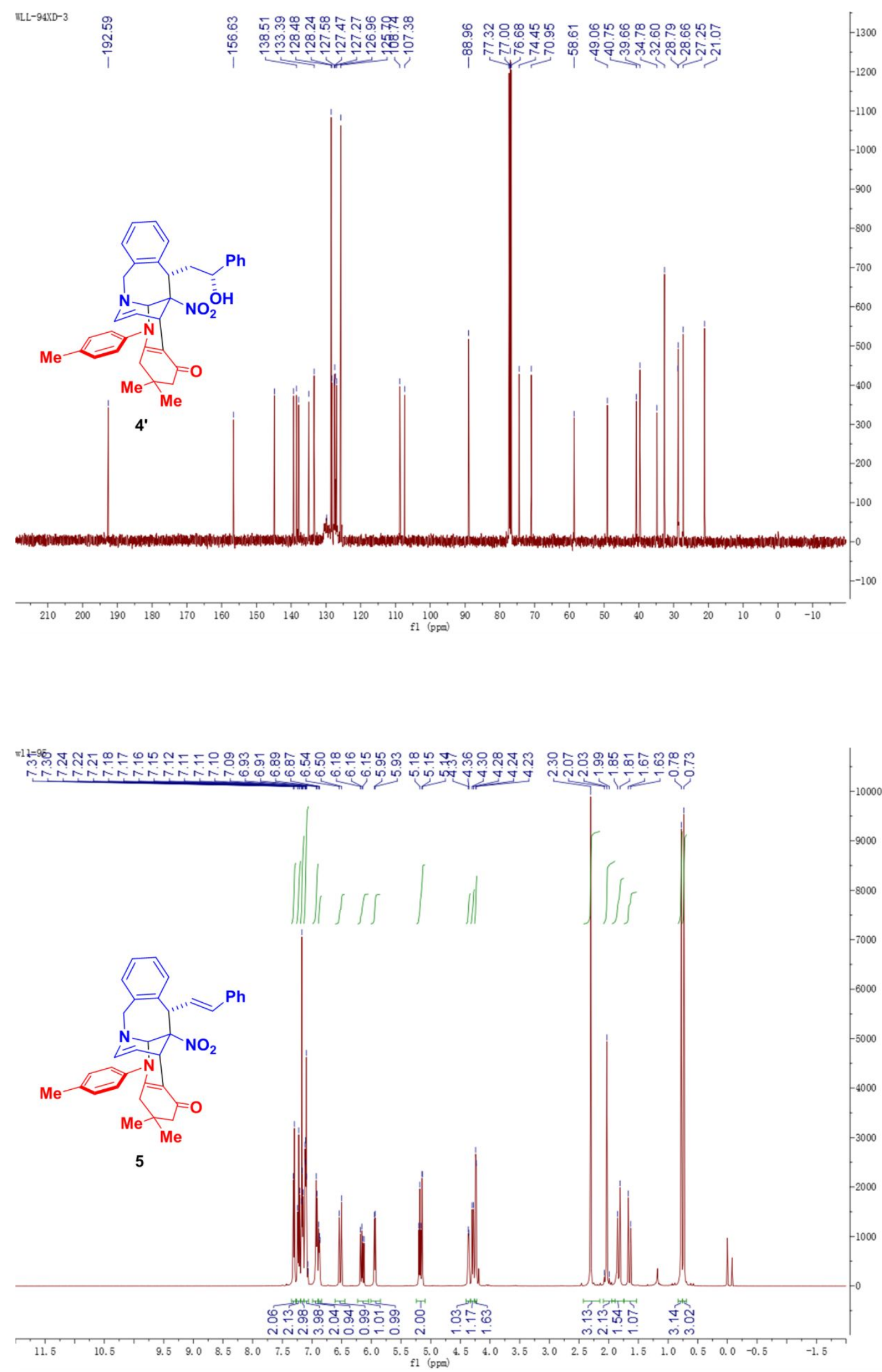

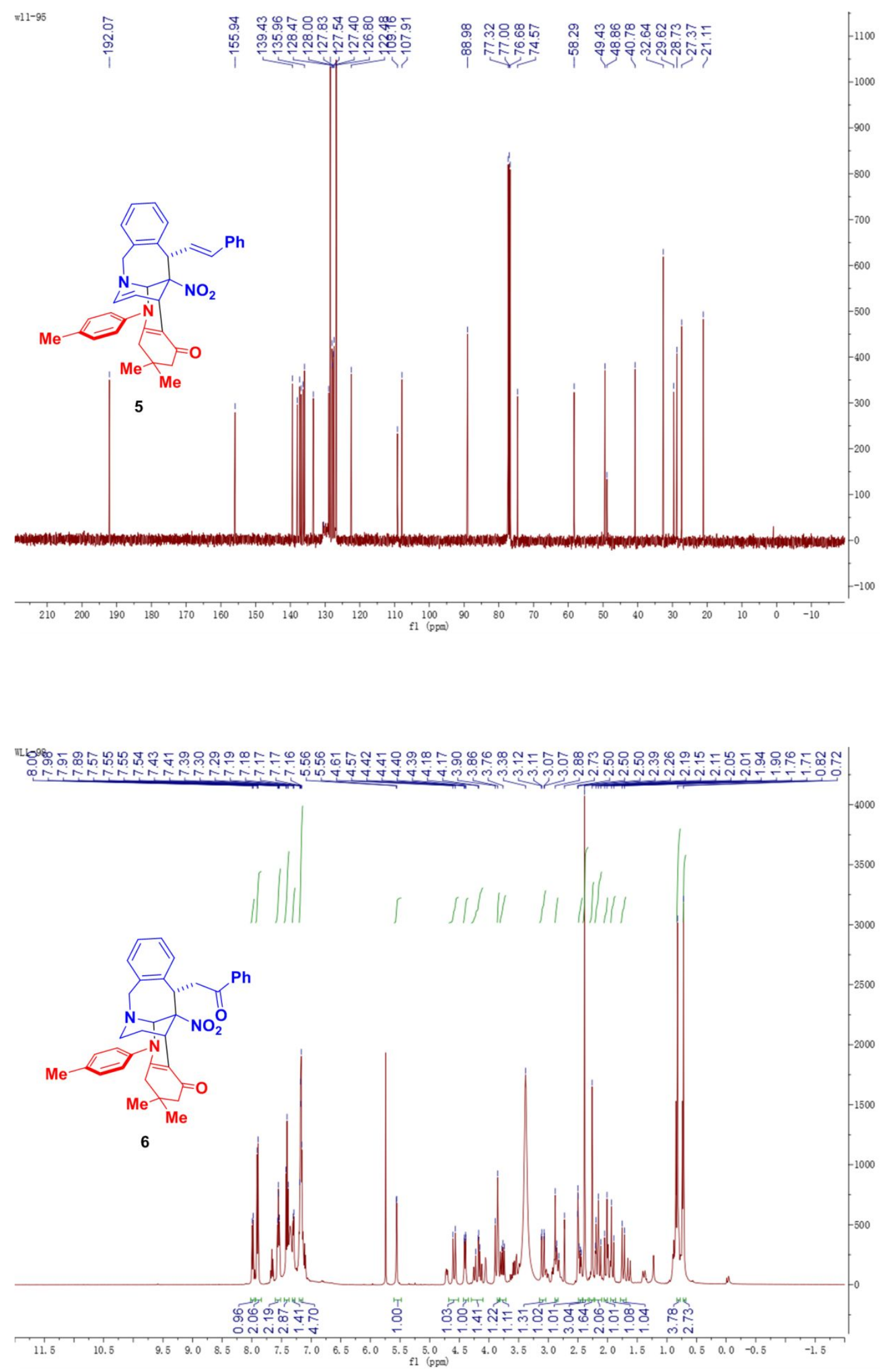


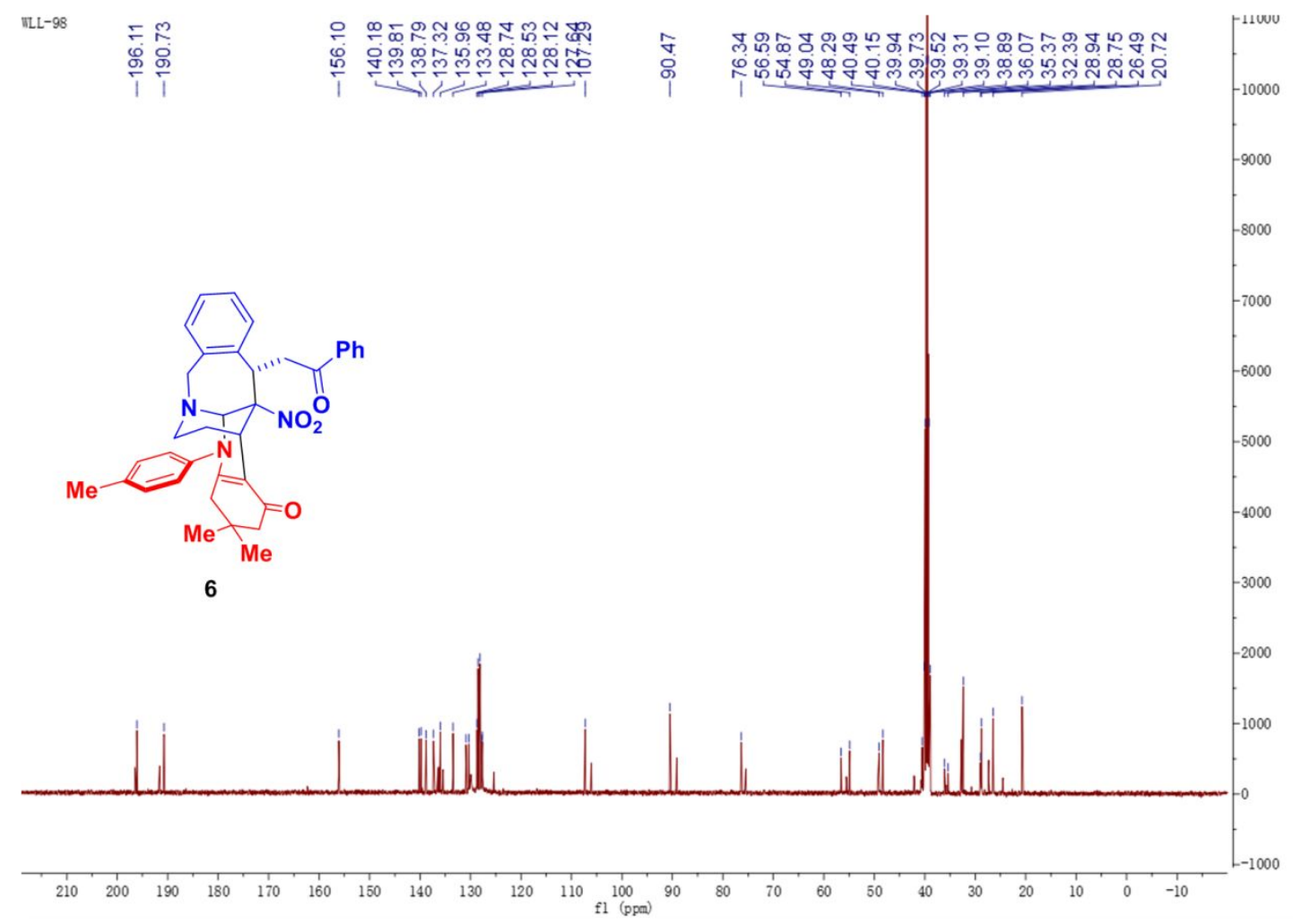

